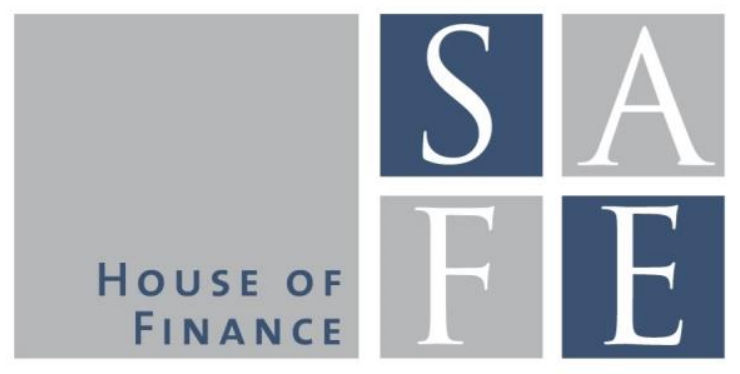

Working PAPER SERIES

Mario Bellia - Loriana Pelizzon - Marti G. Subrahmanyam - Jun Uno Darya Yuferova

\title{
Low-Latency Trading and Price Discovery: Evidence from the Tokyo Stock Exchange in the Pre-Opening and Opening Periods
}

SAFE Working Paper No. 144

SAFE I Sustainable Architecture for Finance in Europe A cooperation of the Center for Financial Studies and Goethe University Frankfurt 


\section{Non-Technical Summary}

We study whether the presence of low-latency traders (including high-frequency traders (HFTs)) in the pre-opening period contributes to price discovery and liquidity provision in the subsequent opening call auction. We compare the behavior of HFTs in the pre-opening period versus the continuous trading period, given that these traders do not have any speed advantage over other traders, in the pre-opening period, on the Tokyo Stock Exchange (TSE). The key reason why there is no speed advantage is that no execution is allowed before the opening call auction on the TSE, which is quite different from other exchanges such as NASDAQ.

Based on a complete, unique dataset of all transactions on the TSE at the millisecond level, we group all traders into 12 groups based on speed of trading and inventory holdings. We find that HFTs dynamically vary their presence in different stocks, and on different days; hence, it is important to distinguish their behavior on a stock-day basis. As SEC (2014) suggests, advanced trading technologies are available and many traders rely on them to formulate their own order submission strategy, in many equity markets today. Our methodology demonstrates that existing studies on HFTs tend to focus on an extremely narrow group of traders and neglect the unique activities of other traders such as algorithmic traders.

We find that in spite of the lack of immediate execution, about one quarter of HFTs participate in the pre-opening period (Type-1 HFTs), and contribute significantly to price discovery. At the same time, they also contribute to liquidity provision in the opening call auction. On the other hand, Type-II HFTs, who constitute three quarters of all HFTs and do not participate in the preopening period, are the most active traders in the first 30 minute of the continuous period, and do play an important role in price discovery and market liquidity. In the continuous period, TypeI HFTs switch their role more to liquidity provision rather than price discovery. Thus, we find that, while HFTs contribute to both price discovery and liquidity provision, there is considerable heterogeneity in their behavior.

Our results suggest that the majority of HFTs prefers trading in the continuous trading period to trading in the opening call auction. This finding points to the need for a careful examination on how a switch from the current continuous auction to a periodic auction, which has been widely discussed in the literature, may affect the behavior of fast traders. Hence, our findings also offer some preliminary evidence in the context of the debate on the relative merits of periodic batch versus continuous auctions. 


\title{
Low-Latency Trading and Price Discovery: Evidence from the Tokyo Stock Exchange in the Pre-Opening and Opening Periods
}

\author{
MARIO BELLIA, LORIANA PELIZZON, MARTI G. SUBRAHMANYAM, \\ JUN UNO and DARYA YUFEROVA*
}

\begin{abstract}
We study whether the presence of low-latency traders (including high-frequency traders (HFTs)) in the pre-opening period contributes to market quality, defined by price discovery and liquidity provision, in the opening auction. We use a unique dataset from the Tokyo Stock Exchange (TSE) based on server-IDs and find that HFTs dynamically alter their presence in different stocks and on different days. In spite of the lack of immediate execution, about one quarter of HFTs participate in the pre-opening period, and contribute significantly to market quality in the pre-opening period, the opening auction that ensues and the continuous trading period. Their contribution is largely different from that of the other HFTs during the continuous period.
\end{abstract}

JEL classification: G12, G14.

Key-words: High-Frequency Traders (HFTs), Pre-Opening, Opening Call Auction, Price Discovery, Liquidity provision.

\footnotetext{
${ }^{*}$ Bellia and Pelizzon are with SAFE, Goethe University and Ca' Foscari University of Venice. Subrahmanyam is with Leonard N. Stern School of Business, New York University. Uno is with Waseda University and Ca' Foscari University of Venice and Yuferova is with Norwegian School of Economics (NHH). We are grateful to Jonathan Brogaard, Austin Gerig, Björn Hagströmer, Joel Hasbrouck, Frank Hatheway, Terry Hendershott, Andrei Kirilenko, Mark van Achter, anonymous high-frequency traders, and participants at the FMA European Conference 2015, the 4th International Conference on the Industrial Organization of Securities and Derivatives Markets: High Frequency Trading, the SAFE Microstructure Workshop, Goethe University, the Conference on Securities Markets Trends, Risks and Policies, CONSOB-BAFFI CAREFIN, Bocconi University, the Swiss Society for Financial Market Research SGF Conference, the Nippon Finance Association Meeting, and the CFS Conference on High Frequency Trading, for helpful suggestions. We also thank the Tokyo Stock Exchange for providing us with anonymous detailed account-level data, which formed the basis of the research reported on in this paper. This work was carried out with the generous financial support of EUROFIDAI, which we appreciate. We also thank the Research Center SAFE, funded by the State of Hessen Initiative for Research (LOEWE), for financial support. Darya Yuferova also gratefully acknowledges the Vereniging Trustfonds Erasmus Universiteit Rotterdam for supporting her research visit to NYU Stern and is also grateful to Rotterdam School of Management, Erasmus University, where some work on this paper was carried out during her $\mathrm{PhD}$ studies.
} 
Global equity markets have been fundamentally altered in the past decade due to vast improvements in the speed of trading and the consequent fragmentation of market activity. Among other changes, in many markets, traditional market makers have been replaced by high-frequency traders (HFTs), operating at the level of a few milliseconds or even microseconds. ${ }^{1}$ This increase in trading speed allows markets to operate far beyond human capabilities, given that the average time it takes for a human to blink varies from 300 to 400 milliseconds. These technological developments have had a dramatic impact on the behavior of liquidity providers and consumers in financial markets, and have implications for price discovery. The resulting changes have led to intense debate and scrutiny from investors, market makers, exchanges, and regulators regarding the advantageous, even unfairly advantageous, status of HFTs in global markets. ${ }^{2}$

Regulators in many countries have been debating, and in some cases have implemented, new regulations on HFTs in recent years. A financial transaction tax has been adopted by France, Italy, and Canada. While there are many aspects of HFT activity and its impact on global equity markets, two issues come to the fore in any policy discussion on the role of HFTs. The first is whether HFTs contribute to price discovery in the sense that they improve the incorporation of new information into asset prices in terms of speed and accuracy. The second is whether they contribute to an improvement in market liquidity, which would permit market participants to enter and exit a position in an asset rapidly and at a minimal cost.

These issues have to be posed in the context of the trading schedules of equity markets. Many equity markets around the world have several distinct periods during the trading day: the pre-opening period, when quotes are placed and trades may or may not occur, an opening call auction, when buy and sell orders are crossed to determine an opening price, and a continuous trading period, when normal trading activity occurs, with posted quotes, orders, and trades. These three periods have different characteristics and the extent of HFT activity may, therefore, vary across them.

In this paper, we aim to investigate the HFTs' ability to contribute to price discovery and liquidity provision in these three different periods. To our knowledge, we are the first to

\footnotetext{
${ }^{1}$ See Brogaard (2010), Jovanovic and Menkveld (2015), Hendershott and Riordan (2013), and Raman and Yadav (2014), for evidence of this phenomenon.

${ }^{2}$ See Lewis (2014) for a popular account of this perspective.
} 
investigate whether HFTs contribute to market quality in the pre-opening period (i.e., when there are no trades) and in the opening call auction (i.e., when speed capacity is irrelevant).

It is important to examine the first two periods before analyzing the continuous period, because the pre-opening period has very different characteristics. The pre-opening period is the first time in the day that information accumulated overnight gets incorporated into market prices, even if only quotes are posted and no trades occur. In contrast, the opening call auction is the first time in the day (after the previous day's closing) that market prices can incorporate new information accumulated overnight, based on actual trades. The continuous trading period, on the other hand, provides a much longer period of trading, but in the context of the arrival of new information, which may affect market fundamentals. HFTs' contributions to price discovery and liquidity may well differ between the three periods. We focus, in this paper, on HFT activity in the pre-opening period and the opening call auction, using the continuous trading period for comparative purposes. Given the growing presence of very fast traders in the market, the manner in which price discovery occurs during the pre-opening period is a crucial issue to be investigated, as is the liquidity provision during the opening auction.

The main questions we address in this paper are related to the role of fast traders, i.e., HFTs, in the pre-opening period, and the difference in trader behavior between this period, on the one hand, and the opening call auction and continuous trading period that follow, on the other. First, we investigate whether, in the absence of trading, HFTs still participate in the pre-opening period and, if they do participate, whether they are more or less active in the pre-opening period than during the continuous period that ensues. Second, we investigate whether HFTs that are present in the pre-opening period contribute to price discovery also in the continuous trading period that ensues. Third, we study whether HFTs provide liquidity in the opening auction, and compare the liquidity provision that occurs with that in the continuous trading period, again distinguishing between HFTs that are present in the opening call auction and those that are present only in the continuous period.

In order to empirically investigate these questions, we use a unique dataset provided by the Tokyo Stock Exchange (TSE), one of the largest stock markets in the world and the market with the largest presence of HFT activity: 55\% compared to $49 \%$ in the U.S. market 
and $35 \%$ in the European market, as of 2013 (as documented by Hosaka (2014)). The TSE is also unique in relation to other major stock exchanges in the world, since it has a market share of over $90 \%$ of all Japanese stocks, while the NASDAQ and the New York Stock Exchange, for example, have a market share of well under $30 \%$ of their markets. Also, our analysis is not affected by information flows of stocks traded in multiple locations, because the TSE trades in a different time zone than the major stock exchanges in Europe and the U.S. (which trade some Japanese stocks directly or in the form of depositary receipts). Finally, our data cover all 1,702 stocks traded on the First Section of the TSE, comprising over $94.8 \%$ of the overall trading volume and $98.6 \%$ of the market capitalization of Japanese stocks, with reliable timestamp data. Our analysis, therefore, refers to virtually the whole Japanese market, and not a subset of it as has been the case in almost every prior study.

At the TSE, the execution of orders is not permitted during the pre-opening period and, hence, buy/sell schedules can be crossed. In fact, traders cannot seek immediacy in this period; therefore, HFTs that have the advantage of moving more quickly than other traders in reacting to new information or order flow cannot employ their superior ability to achieve speedy execution. This may result in almost no presence of HFTs in the opening auction period, although this warrants empirical scrutiny. Therefore, it is interesting to investigate the role played by HFTs during these periods.

A major challenge we face to investigate this question is to identify which traders are HFTs. Our data relate to trading information at the disaggregated level of the virtual servers used for trading. ${ }^{3}$ Based on virtual server usage, we are, therefore, able to infer account-level trading. Hence, we are quite different from prior studies in the microstructure literature, that lack access to such disaggregated data, and are either forced to rely on a HFT/non-HFT flag or, when they do use account-level information, are able to cover only a small sample of the market, and even then, typically focus only on the continuous period.

Moreover, our database permits us to dynamically disentangle the types of trader, on a stock-by-stock, day-by-day, and period-by-period basis. Thus, it is comparable to stud-

\footnotetext{
${ }^{3}$ The study closest in spirit to ours is one by Brogaard, Hagströmer, Norden, and Riordan (2015), who use subscription data for different speeds of co-location services as a screening device for HFTs. They distinguish between traders based on their usage of the low-latency facility, but do not have the relevant information on the server configurations of individual trading desks that we do. Our data are far more granular than theirs and permit a more detailed day-by-day and stock-by-stock analysis.
} 
ies having access to trader identification data and, hence, offers several advantages for researchers that are worth highlighting. First, given the granularity of our data, one can check whether there are differences between trader activity in the pre-opening period, the opening auction, and the continuous trading period that ensues. In turn, our data allow us to measure the impacts of different types of traders on price discovery and liquidity provision. Thus, only with trader identification data one can shed some light on the price discovery and liquidity consequences of HFT activity. Second, our data permit a comprehensive classification scheme, which applies to the trading data on the stock-day-period basis. As we show in our paper, traders tend to switch their type from one day to another, and from one stock to the next; thus, the comprehensive nature of our data allows us to move away from the ad hoc assumption of an immutable HFT classification: "once an HFT, forever an HFT."

Using the granular data available to us, we classify traders into 12 subgroups based on speed and inventory behavior during the continuous period. In terms of speed, we identify three subgroups, namely FAST, MODERATE, and SLOW; in terms of inventory, we identify four subgroups, namely LARGE, MEDIUM, SMALL, and NOTRADE, based on end-of-day inventory. Although these two characteristics, speed and inventory, are generally used in the literature to identify HFTs, they cannot be seen as perfect substitutes. Indeed, it would be better to keep the two dimensions separate in the analysis of the data, which is what we propose to do in our empirical work.

We investigate the issues defined above using the perspective of a policymaker who wishes to assess the benefits of HFT activity in the equity market in the pre-opening period, in the opening call auction and in the continuous period. We find that one quarter of HFTs trading within the continuous period also participate in the pre-opening and opening auction. However, even if they comprise only a minority of HFTs, their participation is highly relevant in terms of the number of quotes they make. We examine the contribution HFTs make to price discovery and find that they do contribute to it during the pre-opening period. Indeed, compared to other types of traders, they are among the groups that contribute the most. We also examine the price discovery contribution made by HFTs in the continuous period and show that, as a group, they contribute to price discovery during that period as well (in line with previous literature, as in Brogaard, Hendershott, and Riordan (2014)). However, the 
subgroup of HFTs that actively participates in the pre-opening section does not contribute to price discovery during the continuous period. This indicates that, while HFTs contribute to price discovery both in the pre-opening and in the continuous period, there is considerable heterogeneity in their contributions by subgroups.

In terms of the HFTs' liquidity contribution during the opening call auction, our analysis shows that HFTs significantly contribute to liquidity provision during the opening call. In line with the previous literature, however, we find that HFTs consume liquidity during the continuous period. The subgroup that actively participates during the pre-opening period, though, contributes to liquidity provision during both the opening call auction and the continuous period. For all three metrics of HFT activity - (i) participation, (ii) price discovery, and (iii) liquidity provision - we perform several detailed analyses, aiming to disentangle the contribution of HFTs conditional on systematic and idiosyncratic volatility. Overall, we find that HFTs do not harm market quality when systematic or idiosyncratic volatility is high.

The outline of the paper is as follows. In Section I, we survey the literature on HFTs, price discovery and liquidity provision. In Section II, we provide a detailed description of the research issues, the hypotheses we investigate, and the empirical methodology we employ. Section III describes the institutional details of the TSE, particularly for HFTs, and the special features of our database. In Section IV, we describe the data-filtering procedures we use to identify the 12 trader groups based on activity during the continuous period. Our empirical analysis and results are presented in Section V. Section VI presents our robustness checks and Section VII concludes.

\section{Literature review}

Our study contributes to the empirical literature on the impact of HFT activity on various measures of market quality, i.e. price discovery and liquidity provision. The recent HFT-specific theoretical literature deals with the speed advantage of HFTs in terms of information processing and trading. Most of it focuses only on the continuous trading session. Their greater speed allows HFTs to react more quickly to public news than other traders

(as in theoretical research by Cespa and Foucault (2011), Pagnotta and Philippon (2011), 
Aït-Sahalia and Saglam (2014), Jovanovic and Menkveld (2015), Budish, Cramton, and Shim (2015), Biais, Foucault, and Moinas (2015) and Foucault, Hombert, and Roşu (2016)). The body of empirical studies on HFT trading activities is growing rapidly. For a review of this burgeoning literature, see Jones (2013) and Biais and Foucault (2014). Studies on HFTs and market quality include Hendershott and Moulton (2011), Hendershott, Jones, and Menkveld (2011), Easley, de Prado, and O'Hara (2012), Hendershott and Riordan (2013), Malinova, Park, and Riordan (2013), Boehmer, Fong, and Wu (2014), Brogaard, Hendershott, and Riordan (2014), Menkveld (2013), Brogaard, Hagströmer, Norden, and Riordan (2015), Gomber, Arndt, Lutat, and Uhle (2011), Menkveld (2013), Chaboud, Chiquoine, Hjalmarsson, and Vega (2014), Hagströmer and Norden (2013), Baron, Brogaard, Hagströmer, and Kirilenko (2016), and Kirilenko, Kyle, Samadi, and Tuzun (2016). The focus of most of this literature is on the continuous trading session, rather than the pre-opening period of the trading day. However, none of these studies describe how HFTs prepare their positions during the preopening period, in anticipation of the continuous trading session, nor do they investigate the behavior of HFTs that carry inventories overnight. In contrast to the prior literature, the particular emphasis of this paper is on HFT behavior in the pre-opening period: If HFTs indeed have superior information-processing ability then it will be advantageous for them to place orders in the pre-opening period as well.

Our research is also related to the microstructure of trading activity in the market preopening period. The pattern of the market pre-opening trading has been studied in the earlier literature (e.g., by Amihud and Mendelson (1991), Biais, Hillion, and Spatt (1999), Cao, Ghysels, and Hatheway (2000), Ciccotello and Hatheway (2000), Madhavan and Panchapagesan (2000), and Barclay and Hendershott (2003)). However, much of this literature is dated, and is based on research conducted well before the rapid growth in the number of HFTs over the course of the past decade or so. It is, therefore, necessary to examine trading activity in the pre-opening period once again, given the dramatic changes that have occurred since the advent of HFT activity. In summary, our paper is related to the previous and current literature on HFTs, but differs in several dimensions. First, it relies on a unique characterization of HFTs that is derived from the specifics of the trading technology (as described in detail in Section IV below), rather than relying merely on trading metrics. Second, 
we use the whole market sample to identify different trader groups on the TSE, while other papers have relied on reasonably complete information, but for a much smaller subset of the market. Our reliance on the identification of server IDs permits us to get around the problem of limited access to client-specific trading data, and yet obtain complete data for the whole market. Third, to our knowledge, there are no papers that investigate the impact of HFT activity on the price discovery process in the pre-opening period and liquidity provision in the opening call auction. Our paper aims to fill this void.

\section{Research issues, hypotheses and methodology}

We take the perspective of a policymaker who wishes to assess the benefits of HFT activity in equity markets. Hence, our perspective is positive: What is the impact of HFTs on the functioning of such markets? ${ }^{4}$ There are two principal potential benefits of such activity for markets: price discovery and liquidity. Price discovery refers to the speed and unbiasedness of the process by which new information, revealed through trading and posting quotes, is incorporated into the market price of an asset. Liquidity refers to the speed and cost of entering and exiting an existing position in the asset in a small time interval.

\section{A. Research issues}

The quoted or traded price of an asset changes in response to the arrival of new information. In addition, a trade or even a quote by one market participant, either in response to the information, or for other reasons such as liquidity, may influence other market participants to change their quotes. Hence, in a market where multiple players are posting quotes, prices are constantly changing for both reasons: information and the trading itself. A natural question that arises with regard to assessing the effectiveness of any market innovation or structural change is whether it influences the speed with which the market price incorporates the twin influences of information and trading, and also whether and how rapidly that price converges to its equilibrium value. While equilibrium values may be difficult to assess in fast-moving

\footnotetext{
${ }^{4}$ We defer normative issues such as the optimal strategy for an HFT, including order placement, size, time, etc., to future research.
} 
markets, with the constant arrival of new information, it may be easier to assess how closely prices adjust to the price that will prevail at a particular point in the future, when several trades are likely to occur. For example, if quotes are being placed in the pre-opening period, a natural question would be whether a quote for a particular asset moves the price towards or away from the price that is going to prevail in the opening call auction. This is the concept of price discovery that we will explore in detail in this paper:

DEFINITION: Price discovery is defined as the effect of a given quote, trade, or type of trader on the deviation of the mid-price from the price that will prevail at a particular point in the future.

A parallel question arises in relation to whether a given quote improves or deteriorates the liquidity in the market, as defined by its impact on immediacy and/or the price impact of the execution of orders. In general, a trade or order that reduces the effective bid-ask spread can be said to improve liquidity. To illustrate, if more quotes or orders are being placed on one side of the market than the other, there is a greater imbalance, and hence a decline in liquidity. For instance, if a particular type of trader decreases this imbalance between demand and supply, she is said to improve liquidity. In that sense, liquidity provision (or consumption) measures the extent to which a particular trader mimics the behavior of a designated market maker (who provides liquidity) or an informed trader (who consumes liquidity). Hence, the definition of liquidity in our context is:

DEFINITION: Liquidity is defined as the effect of a given quote, trade, or type of trader on the reduction in the imbalance between the demand and supply of an asset in the market.

Given these broad conceptual definitions of price discovery and liquidity, we frame the research issues that we seek to investigate:

1. Do HFTs participate in the pre-opening period, despite the fact that no trading occurs during that period of time? If so, what is their level of activity?

2. Do the same HFTs participate in all three time segments, pre-opening, opening call auction, and the continuous periods?

3. Do HFTs participate in the opening call auction and, if so, with what intensity? 
4. What is HFTs' contribution to price discovery in the pre-opening and continuous periods?

5. What is HFTs' contribution to liquidity in the opening call auction and continuous periods?

\section{B. Hypotheses}

We address the above questions in terms of specific empirical hypotheses, which are listed below:

HYPOTHESIS 1: HFTs are the most active participants in the pre-opening, opening call auction, and continuous periods.

This hypothesis establishes that HFTs are significant players in the market.

HYPOTHESIS 2: Of all types of trader, HFTs contribute the most to price discovery in the pre-opening and continuous periods.

Although there is no trading in the pre-opening period, the placement of quotes by the various traders has an impact on price movement during that period. In addition, the opening call auction that follows is a result of the price discovery process during the preopening period.

HYPOTHESIS 3: Of all types of trader, HFTs contribute the most to liquidity in the opening call auction and the continuous period.

Liquidity can be measured only when there is actual placement of orders and trading occurs, and not with the mere posting of quotations. Hence, it is relevant to speak of liquidity only in the case of the opening call auction and the continuous period.

\section{Methodology}

\section{C.1. HFT participation}

The key issue in addressing Hypothesis 1 is the presence of HFTs during the pre-opening section, characterized by the absence of trading. We also aim to test whether they have 
a presence that is larger than that of the other types of trader, and whether a significant proportion of HFTs participate in the pre-opening section. We investigate this hypothesis by looking first at their presence in the pre-opening period, the opening auction, and the continuous period. We define the trader presence ratio as the percentage of traders in trader group $l$ that submit at least one quote for stock $j$, on day $k$, in any of the sections of the day. More formally,

$$
P R_{p, j, k, l}=\frac{\text { Number of trading } \operatorname{desks}_{p, j, k, l}}{\sum_{p} \text { Number of trading } \operatorname{desks}_{p, j, k, l}}
$$

where $p$ is the period considered (pre-opening, opening auction, continuous) for stock $j$, on day $k$, for the trader group $l$. Therefore, the Number of trading desks $s_{p, j, k, l}$ is the number of traders in trader group $l$ that submit at least one quote for stock $j$ on day $k$ in period $p$.

We then investigate their presence in the pre-opening (and the continuous) period using the activity ratio based on the number of quotes:

$$
Q A R_{p, j, k, l}=\frac{\text { Number of quotes }_{p, j, k, l}}{\sum_{l} \text { Number of quotes }_{p, j, k, l}}
$$

where $p$ is one of the two periods considered, pre-opening or continuous (there are no quotes in the opening auctions, only trades), and where the ratio relates to stock $j$, day $k$, and trader group $l$.

For the opening auction, we calculate the activity ratio by considering the number of shares traded, instead of the number of quotes:

$$
T A R_{p, j, k, l}=\frac{\text { Number of shares } \operatorname{traded}_{p, j, k, l}}{\sum_{l} \text { Number of shares } \operatorname{traded}_{p, j, k, l}}
$$

where $p$ is one of the periods considered (opening auction or continuous), for stock $j$, day $k$, and trader group $l$. We calculate this other ratio for the continuous period as well. 


\section{C.2. Price discovery}

The key issue in addressing Hypothesis 2 regarding price discovery is the informativeness of trading by HFTs. We investigate which trader groups contribute to the price discovery process, and compare the extent of their contributions using order-by-order (trade-by-trade) data and associated quoted (traded) price changes. In this manner, we take advantage of our detailed data, as we can pinpoint an order (trade) that moves the quoted (traded) price and, thus, we can identify which trader group submitted that order (initialized that trade) and the type of the order. We measure the amount of new information incorporated into stock prices using the weighted price contribution $(W P C)$ suggested and used also by Barclay and Warner (1993), Cao, Ghysels, and Hatheway (2000), and Barclay and Hendershott (2003). The $W P C$ measures how much the prices deviate from the price that will prevail at a later date, for instance between the pre-opening period and the opening call auction. We apply the same methodology for both the pre-opening and the continuous period. Specifically, for the continuous period we concentrate our analysis only on the first thirty minutes because we wish to compare the behavior of traders between the pre-opening and the continuous period with a minimal effect from new information.

First, we define the price discovery contribution as the amount by which an incoming order (trade) moves the quoted (traded) price closer to the reference price. Thus, we compute the price discovery contribution $(P D C)$ on an order-by-order (trade-by-trade) basis, as follows:

$$
P_{i, j, k}=\text { Deviation }_{i, j, k}-\text { Deviation }_{i-1, j, k}
$$

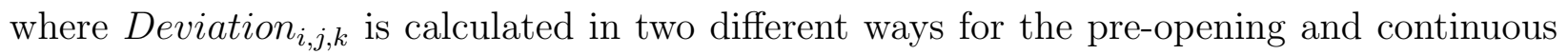
periods, but with a reduction in the deviation being viewed as price discovery.

For the continuous period, the deviation of the trading prices from the price at 9:30 a.m. is calculated as

$$
\text { Deviation }_{i, j, k}=\left|\frac{P_{i, j, k}}{P 930_{j, k}}-1\right| \times 100
$$

where $P_{i, j, k}$ is the trading price at the time of the $i$-th transaction, for stock $j$, on day $k$, and $P 930_{j, k}$ is the price at 9:30 a.m. for stock $j$ on day $k$. In order to determine the price during 
the first 30 minutes of continuous trading, we use the average trading price between 9:30 and 9:35 a.m. to avoid the bid-ask bounce problem. Deviation ${ }_{i, j, k}$ in this case is the absolute deviation from the price at 9:30 a.m., of the traded price at the time of the $i$-th trade, for stock $j$ on day $k$ (see equation (5)). Similarly, Deviation ${ }_{i-1, j, k}$ is the absolute deviation from the price at 9:30 a.m., of the traded price at the time of the $(i-1)$-th trade, for stock $j$ on day $k$.

The amount by which Deviation $_{i, j, k}$ is lower than Deviation De $_{i, j, k}$ is the contribution to price discovery made by the order that initiates the $i$-th trade. Orders that initiate the transaction are new market orders and new or revised limit orders that either lock in or "cross" the prevailing bid-ask spread. ${ }^{5}$ We discard those transactions from the continuous trading period for which we cannot identify the initiating order. ${ }^{6}$ We refer to orders that initiate a transaction as "aggressive orders" (as in Biais, Hillion, and Spatt (1995), Ranaldo (2004), Duong, Kalev, and Krishnamurti (2009), and Yamamoto (2011)). For the preopening, Deviation $_{i, j, k}$ is defined as

$$
\text { Deviation }_{i, j, k}=\left|\frac{M_{i, j, k}}{O_{j, k}}-1\right| \times 100
$$

where $M_{i, j, k}$ is the quoted price at the time of arrival of order $i$ for stock $j$ on day $k$, and $O_{j, k}$ is the opening price for stock $j$ on day $k$.

Deviation $_{i, j, k}$ in this case is the absolute deviation of the quoted price from the opening price, immediately after order $i$ is entered for stock $j$ on day $k$ (see equation (6)). Deviation $_{i-1, j, k}$ is the absolute deviation of the quoted price from the opening price, immediately before order $i$ is entered for stock $j$ on day $k$. The amount by which Deviation $n_{i, j, k}$ is lower than Deviation $_{i-1, j, k}$ is the contribution to price discovery made by order $i$.

Among the orders submitted during the pre-opening period, we can identify those orders with the potential to impact the prevailing quotes. We call them "aggressive orders" (as

\footnotetext{
${ }^{5}$ Locked limit orders are orders with the limit buy (sell) price equal to the best ask (bid) price, while crossed limit orders are orders with limit buy (sell) price greater (smaller) than the best ask (bid) price (see Cao, Ghysels, and Hatheway (2000)).

${ }^{6}$ If an order imbalance causes a larger price change than the pre-specified amount (e.g., the maximum price change between two trades is 70 Japanese Yen in the price range 3000-5000 Japanese Yen), the TSE stops continuous trading and conducts a call auction. The TSE disseminates special quotes to notify the market about the trading halt. In our sample, less than $1 \%$ of the trades fall into this category.
} 
for the continuous period described above). The TSE uses unique rules for determining the best pre-opening bid and ask quotes. These rules are different from those applied in the continuous period and are briefly explained in Section III and in the Internet Appendix A. There are four cases in which we categorize an order as aggressive: (1) all market orders; (2) a limit buy order with a limit price greater than or equal to the prevailing best bid; (3) a limit sell order with a limit price less than or equal to the prevailing ask; and (4) any orders submitted at a time when the best bid equals the best ask. ${ }^{7}$ When $P D C_{i, j, k}$ as defined in equation (4) is negative, the deviation is reduced and the quoted (traded) price moves closer to the reference price. We define the $W P C$ for stock $j$, day $k$, and order $i$ as

$$
W P C_{i, j, k}=\frac{P D C_{j, k}}{\sum_{j}\left|P D C_{j, k}\right|} \times \frac{P D C_{i, j, k}}{P D C_{j, k}}
$$

where $P D C_{i, j, k}$ is the price discovery contribution of order (trade) $i$, for stock $j$, on day $k ; P D C_{j, k}$ is the accumulated price discovery contribution for stock $j$, on day $k$. The first term of $W P C$ is the weighting factor for the stock $j$ on day $k$. The second term is the percentage contribution made by order (trade) $i$ to the total price discovery, during either the pre-opening or the continuous period, for stock $j$ on day $k$. Since the size of $P D C$ varies for each stock and each day, the relative contribution adjusts for the scale difference across stocks as well as across trading days, while the first factor adjusts for the relative importance of price discovery across stocks on day $k$. When $P D C_{j, k}$ equals zero, we do not compute $W P C$ for stock $j$ on day $k$. We also winsorize $P D C_{i, j, k}$ at the $0.1 \%$ and $99.9 \%$ levels.

There are several approaches in the existing literature, for measuring price informativeness. For example, Hasbrouck (1995) develops a methodology for estimating information shares. In addition, Van Bommel (2011) summarizes three prominent alternatives that have been used widely in the literature: (1) the variance ratio; (2) the $R^{2}$ of the regression of unbiasedness of prices; and (3) the weighted price contribution $(W P C)$, which is the one

\footnotetext{
${ }^{7}$ Such a situation occurs when the cumulative amount of buy orders equals that of sell orders. Thus, the next order must cause an imbalance between buy and sell orders, and make the best ask higher than the best bid price. We refer to such orders as "locked orders." Cao, Ghysels, and Hatheway (2000) analyze locked/crossed market quotes during the NASDAQ pre-opening period. In the TSE's pre-opening period, the market best quotes may be locked, which means that the best ask equals the best bid, but crossed quotes (which means that the best bid is greater than the best ask) never happen, by rule.
} 
used in this paper. In simple terms, the variance ratio compares the variances in, say, the pre-opening period and the continuous period, and the test looks at whether that ratio is equal to one. The $R^{2}$ of the unbiasedness regression tests, for example, whether the evolution of prices in the pre-opening period is an unbiased predictor of the prices in the call auction. Van Bommel (2011) argues that, if the price process is a driftless martingale, only the W PC is an unbiased estimator for the return variance explained during a time interval. On top of this, we prefer using the WPC methodology to the other metrics for a variety of reasons. First, we do not take a position on price efficiency or the long-term fundamental value of the asset. Rather, we measure the deviation with respect to the price observed later, at a given point in time. Our concept of price discovery is, therefore, different from the information share measure developed by Hasbrouck (1995) and Hasbrouck (2002), which requires the notion of an unobservable efficient price. In fact, thanks to the granularity of our data to identify the actions of individual traders, we are not constrained to merely document a statistical relationship between trading intensity and subsequent price movements, but are able to provide a detailed analysis of how each action by a given trader can move the price, all other influences being held fixed.

\section{C.3. Liquidity}

Since our data provide information at the millisecond level on trade size, trade direction, and the initiator of the trade, we can study, in detail, for each trade, whether liquidity is provided or consumed, and by which trader. There are several alternative proxies that could be used to analyze market liquidity, but for our purpose here, we define liquidity provision (consumption) depending on whether a trader acts like a market maker (taker). In other words, during the continuous period, a liquidity provider is one that posts orders that do not initiate trades: orders that are not market orders or marketable limit orders. We look, in detail, at all such orders and calculate for each trader group, each trade, each stock, and on each day, the following liquidity provision (LP) and liquidity consumption (LC) ratios for the continuous period:

$$
L P_{i, j, k, l}=\frac{\text { Number of shares traded }{ }_{i, j, k, l} \mid \text { Trader }_{l} \text { does not initiate trade }}{\text { Total traded volume of first } 30 \text { minutes of continuous period }_{j, k}}
$$




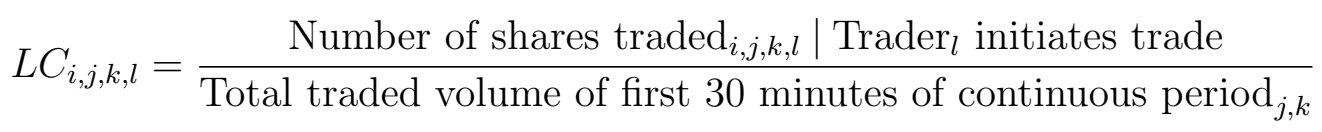

where $L P$ represents the liquidity provision and $L C$ the liquidity consumption of trade $i$ for stock $j$ on day $k$. We then define the net liquidity provision for the continuous period by calculating the difference between the two ratios, that is

$$
N L P_{i, j, k, l}=L C_{i, j, k, l}-L P_{i, j, k, l}
$$

We calculate a similar measure for the opening call auction. In this case, we assume that the trader is a liquidity provider when she is trading in the opposite direction of the market. Otherwise, she is a liquidity consumer. We define the direction of the trade by analyzing the difference between the opening call auction price $P_{a}$ and the closing price of the day before $P_{c}$. When $P_{a}>P_{c}\left(P_{a}<P_{c}\right)$, a selling (buying) trade is trading against the market and is therefore providing liquidity, and a buying (selling) trade is demanding liquidity. Based on this definition, we calculate, for the opening call auction,

$$
\begin{gathered}
L P_{i, j, k, l}=\frac{{\text { Number of } \text { shares }_{i, j, k, l} \text { traded against the market }}_{\text {Total traded volume of the } \text { auction }_{j, k}}}{L C_{i, j, k, l}=\frac{\text { Number of } \text { shares }_{i, j, k, l} \text { traded in the same direction as the market }}{\text { Total traded volume of the auction }_{j, k}}}
\end{gathered}
$$

We then calculate the net liquidity provision for the opening call auction by calculating the difference between the two ratios, as in equation (10) above.

\section{C.4. Testing methodology}

In the previous subsections, we have defined the different measures we employ to investigate the three hypotheses. For these measures, we report their averages and test whether

these ratios are statistically different to those of the other trader groups by estimating the following panel regression:

$$
Y_{p, j, k, l}=a_{0, p}+\sum a_{l, p} * I_{l}+e_{p, j, k, l}
$$


where $Y_{p, j, k, l}$ is the investigated measure, that is, participation measured by $Q A R$ or $T A R$, price discovery by $W P C$, and liquidity provision by NLP. $p$ is the period considered (preopening, opening auction, continuous) for stock $j$, on day $k$, for trader group $l$. $I_{l}$ is a dummy variable that equals 1 for trader group $l$. We then test whether the coefficients $a_{l}$ are statistically different from zero, and statistically different between one trader group and another.

We also perform a second test for whether (i) the HFT presence, (ii) the price discovery contribution, and (iii) the liquidity provision are statistically larger than all the other trader groups considered as non-HFTs, in line with the analysis presented in the previous literature. More formally, we estimate the following regression:

$$
Y_{p, j, k, l}=a_{0, p}+a_{H F T, p} * I_{H F T}+e_{p, j, k, l}
$$

where $p$ is the period considered (pre-opening, opening auction, continuous), for stock $j$, on day $k$, for trader group $l$, and $I_{H F T, p}$ is a dummy variable that equals 1 , for the trader group that we identify as HFT using the methodology described below.

\section{Institutional structure and data description}

\section{A. Institutional structure of HFT on the TSE}

The trading schedule for the TSE is characterized by two separate trading periods, one in the morning and one in the afternoon, along with two pre-opening periods (one early in the morning and the other in the middle of the day). ${ }^{8}$ Figure 1 provides a representation of the different trading periods. In each trading period, morning and afternoon, the opening price is determined by a single price auction ("Itayose" in Japanese) that kicks off at 9:00 a.m. in the case of the morning session, and at 12:30 p.m. in the case of the afternoon session, based

\footnotetext{
${ }^{8}$ In Japan, there are two private venues, SBI Japannext and ChiX-Japan, whose trading periods start before 9:00 a.m.; however, very little trading occurs before 9:00 a.m. It should be mentioned that, for the Nikkei Stock Index Futures, traded on the Singapore Stock Exchange (SGX) in Singapore, trading starts at 8:45 a.m., Tokyo time, and such trading may also contribute to price discovery.
} 
on buy and sell orders accumulated during the pre-opening periods that precede them. ${ }^{9}$

\section{[Place Figure 1 about here]}

The opening time on the TSE is not randomized, as happens on some European exchanges such as Deutsche Bourse. As soon as the opening price of a stock is determined, the continuous trading period for the stock commences. Thus, these three-periods segments, the pre-opening, the opening call auction, and the continuous trading periods, are connected seamlessly. In the pre-opening period, investors are free to submit orders as they do in the continuous period. There are two general types of orders allowed on the TSE: limit orders and market orders. ${ }^{10}$ Each trading day, the TSE starts receiving orders from its memberbrokers at 8:00 a.m. (12:05 p.m. for the afternoon session), and does so without executing any orders until the single price auction for the market opening begins, at 9:00 a.m. (12:30 p.m.).

As soon as the TSE receives orders, it disseminates the pre-opening quotes, not just the best ask and best bid, but 10 quotes above and below the best quotes, to the market. ${ }^{11}$ Every time the exchange receives an order, the pre-opening quotes are refreshed. The best ask (bid) is identified as the smallest (largest) ask (bid) price at which the cumulative depth of the ask (bid) schedule is greater than the cumulative depth of the bid (ask) schedule. A detailed description of the determination of the best bid and ask, and the rules are reported in the Internet Appendix A. The principle underlying the order matching is based on price and time priority in the continuous period. In the opening call auction, however, time priority is ignored. That is, all orders placed in the pre-opening period, before the opening price has been determined, are regarded as simultaneous orders.

The opening auction determines the price at which the largest number of executions (in terms of number of shares) is possible. There are three conditions to be met at the auction:

\footnotetext{
${ }^{9}$ Single price auctions are used to determine closing prices as well. The rules for the establishment of the closing call auction price are slightly different from those for the opening call auction price. See TSE (2015).

${ }^{10}$ Traders can specify that an order only be eligible for execution at the opening auction. Should it not be executed at the opening auction, such an order will be cancelled automatically, rather than being moved to the continuous trading period.

${ }^{11}$ A subscriber to the "full quotes" service would be able to see information (price and quantity) on the entire order book. However, the quantities for the best ask and the best bid will be the same as for the standard service.
} 
(1) All market orders must be executed at the opening price. (2) Orders with a sell limit price lower than the opening price, and buy limit price higher than the opening price, must be executed. (3) Buy and sell orders with limit prices equal to the opening price must be executed for the entire amount on either the buy or the sell side. The third condition suggests that, often, orders on either side, whose limit prices are equal to the opening price, are not fully executable. If this happens, the TSE allocates the available shares to participating member firms on a pro-rated basis. ${ }^{12}$ As explained above, the speed (or timing) of order submission does not matter during the pre-opening period because of the lack of time priority in the opening call auction. Therefore, investors care about the timing of their submission only due to the third condition above, and the treatment of unfilled orders in the continuous period, when time priority is activated. It should be noted that the feature of the opening call auction, whereby there is no time priority for limit orders submitted during the preopening period, can cause delayed order submissions, price revisions, and cancellations, until just before market opening.

\section{B. Virtual server IDs and data}

We use order data covering the complete history of an order (new order entry, execution, revision of quantity or price, and cancellation in the pre-opening and continuous trading periods) obtained from the TSE. Each historic record is time-stamped at the millisecond level, and includes information on order type, side (buy or sell), number of shares, limit price, unique order number, and virtual server ID. We also use data on the filled orders, and unfilled orders (cancelled immediately) for the opening call auctions, during the period of our study, between April 1 and May 31, 2013. We focus on April and May 2013 since the volatility of the stock market rose around that time, after the new governor of the Bank of Japan, Haruhiko Kuroda, announced a new aggressive quantitative easing (QE) policy for the bank. Given the structural change in the regime, a number of unexpected events occurred during this period, rendering the role of the pre-opening quotes more crucial than at any other time.

The unique feature of this study is that we use novel data provided by the TSE, which

\footnotetext{
${ }^{12}$ For further details of pro-rated allocation refer to TSE (2015, pp. 28-20).
} 
include the unique IDs of the VS. ${ }^{13}$ We find that 5,580 VS were used in the sample period considered. However, we observe that orders pertaining to the same stock are sometimes submitted by different VS; for example, an order submitted by VS "A" was cancelled by VS "B." To determine the relationship between VS, we therefore investigate the entire universe of stocks traded on the TSE's First Section in the period considered, that is all 1,702 stocks. ${ }^{14}$ By combining all the order and trade information from the market in our sample period, we identify 3,021 groups of VSs which we call traders because they represent trading desks. Figure 2 depicts the sizes of the traders based on the number of VS they employ. Among the 3,021 traders, 329 utilize between two and 41 VS, while the rest $(2,692)$ use only a single VS. ${ }^{15}$

\section{[Place Figure 2 about here]}

Thanks to our matching procedure, our database is similar to the one with the anonymous trader identifiers, because we are able to track the traders' behavior through time. To our knowledge there are only few studies in the HFT literature that use a database with user/trader identifier: Hagströmer and Norden (2013), Malinova et al. (2013), Menkveld (2013), Brogaard et al. (2015), Baron et al. (2016), and Korajczyk and Murphy (2015). The data typically come from Nordic (NASDAQ OMX) or Canadian (Toronto Stock Exchange) markets, with the exception of Menkveld (2013) who focuses on the Dutch market. However, market fragmentation poses an important limitation for the abovementioned studies and, in particular, for the coverage of platforms on which a particular stock is traded. Even if the data cover not only regulated exchanges, but also alternative trading venues, such additional data are limited to the country under investigation, thus excluding, for instance, the data regarding traders' behavior in respect to Canadian stocks being traded in U.S. markets. Moreover, even if information from different platforms is available, it is not a trivial task to

\footnotetext{
${ }^{13}$ The Virtual Server (VS) is the logical path established between TSE's system (matching engine) and a user's system in order to communicate with each other. Internet Appendix B describes the typical setup of multiple VSs used by one trader.

${ }^{14}$ Stocks listed on the TSE are split into different sections based on their market capitalization, the number of shareholders, and other parameters. The First Section of the TSE includes relatively large companies, and comprises about $94.8 \%$ of the overall trading volume and $98.6 \%$ of the market capitalization.

${ }^{15}$ In contrast to Brogaard, Hagströmer, Norden, and Riordan (2015), who use the grade of the co-location service as a categorizing device for measuring the speed requirements of traders, we focus instead on how traders configure their respective trading environments.
} 
synchronize the time stamps of trades and quotes across different venues, which makes it virtually impossible to analyze which particular order moved a particular price. In contrast to the Nordic and Canadian equity markets, the Japanese market exhibits a very low degree of fragmentation. In particular, only $6.5 \%$ of the total trading volume in 2013 was hosted by private venues other than the TSE. ${ }^{16}$ Besides that, when the Japanese market is open, most major markets in North America and Europe are closed.

Last but not least, the Japanese market is a bigger market than the Nordic and Canadian ones and, thus, likely to attract more HFT activity. In fact, as mentioned above, according to Hosaka (2014) the TSE market has an HFT presence of 55\% of trades, compared to $49 \%$ in the U.S. and $35 \%$ in Europe. All in all, our data have an important advantage over the data previously used in the literature as they allow us to avoid issues that arise from market fragmentation and therefore allow us to provide a more complete picture of HFT activity. To our knowledge, we are also the first to utilize trade/quote data with trader identifiers to analyze the pre-opening period. It should be stressed that our data are composed of all the stocks in the TOPIX100, the index of major stocks, in Japan. Additionally, we do not have concerns about market segmentation.

Table I presents the characteristics of the traders, based on the trading environment of the 1,702 stocks in the TSE's First Section. As the table shows, the median number of stocks traded per day, per group of VSs, ranges from 183 when only one VS is used, to 963 when $40 \mathrm{VSs}$ are used. The median number of stocks traded, per VS, decreases monotonically from the group that uses only one VS (183 stocks) to the group that uses 20-29 VSs (16 stocks). Increasing the number of VSs beyond 30 leads to an increase in the number of stocks allocated to each VS. We also investigate the maximum speed capabilities of the different traders as well as the end-of-day inventories. We measure the speed as the minimum time that elapsed between two consecutive order submissions for the same stock. Traders with just a single VS place orders with a median speed of 12 seconds, and a median inventory of $100 \%$. These characteristics match those of retail and wholesale brokers, which typically have several buy-side customers. For traders that use multiple VSs, as the number of VSs

\footnotetext{
${ }^{16}$ Chi-X Japan accounts for $2.7 \%$ of market share, while Japannext PTS accounts for roughly the $3.8 \%$ of the market share. See Chi-X (2013) and Japannext (2013).
} 
used by a trader increases from two to 41, the median speed moves to one millisecond; therefore, as soon as the number of VSs used increases, speed increases as well. The median inventory varies considerably across traders, but is negatively related to the number of VSs used, reflecting the variety of investment horizons among them, and indicating that traders with higher speeds usually hold less inventory at the end of the day. In the TSE, some traders, such as HFTs, use multiple VSs exclusively because of a limitation on the number of quotes submitted per second for each VS. ${ }^{17}$ As the table shows, by using multiple VSs, the traders are able to increase their trading speed significantly.

\section{[Place Table I about here]}

\section{The universe of stocks and the sample period}

For our analysis on market quality, we limit the sample of stocks to the constituents of the TOPIX100 index, during April and May 2013. This index is comprised of stocks from the TSE's First Section, with high liquidity and relatively large market capitalization, and comprises over $60 \%$ of TSE market capitalization. Of the TOPIX100 stocks, we exclude three that have larger trading volumes in exchanges other than the TSE, since the focus of our study is the trading system on this exchange. ${ }^{18}$

In our analysis, we exclude stock-days for which special quotes are disseminated before or during the single price auction, because orders submitted during the pre-opening period do not meet the normal opening price rules. For the purpose of our analysis, we concentrate on three periods: the pre-opening section excluding the first 10 minutes, the opening auction, and the first 30 minutes of the continuous period. We exclude the first 10 minutes of the pre-opening period because during the first 10 minutes, the limit order book accumulates

\footnotetext{
${ }^{17}$ The TSE provides three levels of service, with a maximum of 60,40 , and 20 quotes per second, respectively. We refer to all messages, including cancellations, in our definition of quotes. According to a prominent HFT, for a trader that wishes to be truly anonymous, at least $20 \mathrm{VSs}$ are necessary, in order to implement a strategy of trading 1,500 stocks all at once. If the HFT also needs to cancel several orders immediately after submitting new ones, an additional $20 \mathrm{VSs}$ may be required, making a total of $40 \mathrm{VSs}$ necessary to support intensive HFT activity across multiple stocks. Using multiple VSs, each trader optimizes the performance of the trading operations for their subset of stocks. Some traders operate within a specific group of stocks every day, in which case they may fix the allocation of stocks to each VS. Other traders may change part of their allocation on a day-by-day basis.

${ }^{18}$ The three excluded stocks are Murata, Nintendo, and Nihon Densan.
} 
many orders that were waiting overnight for the beginning of the pre-opening period. The arrival times of these orders are not directly related to the traders' actual submission decisions. Therefore, in our analysis of the pre-opening period, we focus on the remaining 50 minutes, during which traders monitor pre-opening quotes and make order submission decisions accordingly. ${ }^{19}$

We focus on the first 30 minutes of the continuous trading period, as we wish to analyze the difference in trader behavior, based on the same information, but in different trading periods: the pre-opening period, the opening call auction, and the continuous trading period. If we extended the sample to the full continuous trading period, we would contaminate our analysis with new information arriving in the market later in the trading day, and harm the comparability with the other periods.

\section{HFT identification}

The data we have allow us to (i) track trader behavior, (ii) cluster traders into several different groups and, in particular, (iii) identify HFTs.

\section{A. HFT identification strategy}

A useful guideline defining the features of HFTs has been presented by the U.S.'s Securities and Exchange Commission (SEC). The document, (SEC, 2010, p. 45), lists five characteristics of HFTs:

1. "Use of extraordinarily high speed and sophisticated programs for generating, routing, and executing orders."

2. "Use of co-location services and individual data feeds offered by exchanges and others to minimize network and other latencies."

3. "Very short time-frames for establishing and liquidating positions."

4. "Submission of numerous orders that are cancelled shortly after submission."

\footnotetext{
${ }^{19}$ The inclusion of the first 10 minutes heavily affects the $W P C$, measures. However, the role of HFTs is qualitatively similar, if we include the first 10 minutes in the analysis.
} 
5. "Ending the trading day in as close to a flat position as possible (that is, not carrying significant, unhedged positions overnight)."

Motivated by this list of characteristics, we use both speed and inventory to classify traders. These two metrics are closely related to all five characteristics listed above: speed matches characteristics 1, 2, and 4 above, while inventory matches characteristics 3 and 5 . The speed is largely determined by the trading infrastructure in which each trading desk invests (the number of VSs, the software programs used, the quality of servers installed, etc.), and which is not easily replaceable in the short run, whereas inventory is closely related to trading styles, such as those exhibited by buy-side investors, market makers, and arbitrageurs.

With these two measures in mind, we are able to investigate how the different traders' behavior affects the pre-opening period. One issue we have to address in our classification is whether the members of different groups of traders remain the same across time and stocks. To our knowledge, HFTs engage in a variety of strategies that do not necessarily remain the same from one day to the next or across stocks. In fact, HFTs implement multiple algorithms depending on whether they believe that the liquidity-taking or the liquidity-making strategy offers more profitable opportunities for a given stock on a given day. Therefore, we can assume that traders can engage in different types of trading strategies on a stock-by-stock and day-by-day basis. Keeping this type of strategic behavior in mind, we compute our metrics on a per-stock, per-day basis, for all trading desks.

\section{A.1. Speed}

We empirically measure the minimum elapsed time between two consecutive order submissions for the same stock, without any restrictions, for a combination of two order types (i.e., any two out of new orders, cancellations, and revisions during our sample period), as a measure of speed. ${ }^{20}$ We refer to our measure of time elapsed, using the time stamp of the order, as speed. In other words, a high speed means a short elapsed time between order submissions. Some authors refer to this concept as latency, but we prefer to use speed since

\footnotetext{
${ }^{20}$ Hasbrouck and Saar (2013) measure low-latency activity by identifying "strategic runs," which are linked submissions, cancellations, and executions that are likely to be part of a dynamic strategy. However, their data do not enable them to identify individual traders as we can.
} 
we take latency to have connotations of the technological limits of speed, rather than the actual speed realized. Henceforth, we simply call our measure "speed".

A realization of a very high speed has to be supported by the appropriate trader's infrastructure. Hence, the number of VSs a trader uses is a crucial determinant of her speed. As noted earlier, we observe varying numbers of VSs, ranging from one to 41, used by the same trader, in our sample period. We also find that the number of stocks allocated to an individual VS is negatively associated with the speed of the trader and vice versa. ${ }^{21}$

\section{A.2. Inventory}

The other major classification variable we employ is the inventory of the trader. Trader inventory is estimated as the (absolute) ratio of the buy volume minus the sell volume at the end of day $k$, divided by the total trading volume of the trader on that day. Many empirical studies report that the key characteristic of HFT liquidity providers is a flat inventory position at the end of each trading day (Menkveld (2013), Kirilenko, Kyle, Samadi, and Tuzun (2016), and SEC (2014)). To investigate this issue further, we compute the end-ofday inventory for each trader and for each stock, as well as intraday inventory, volatility of intraday inventory, and the number of times during the day that the inventory crosses zero, per trader-stock-day.

\section{A.3. Classification}

We classify all traders according to observed speed and inventory during the continuous trading period for each stock-day. We apply the following classification scheme: We divide all traders, based on their speed, into three groups: FAST, MODERATE, and SLOW. For each stock-day, the SLOW group includes traders with a speed greater than 60 seconds. We then split the remainder of the speed distribution relative to the median per stock per day. Therefore, the FAST group includes traders whose speed is less than the median, and the MODERATE group includes traders whose speed is greater than the median, but smaller than or equal to 60 seconds. Where we are unable to compute the speed due to the absence

\footnotetext{
${ }^{21} \mathrm{~A}$ detailed analysis of the relationship between speed and quotes per VS is provided in the Internet Appendix C.
} 
of multiple orders for the same stock on the same day, we treat the trader as a SLOW trader. Therefore, our definition of speed is not nominal speed but relative speed. This is in line with what Baron, Brogaard, Hagströmer, and Kirilenko (2016) find, that it is relative speed, and not nominal speed, which drives differences in performance across HFTs: it is not being fast that allows a HFT to capture trading opportunities but being the fastest. This is also in line with the theoretical literature on HFTs: Biais, Foucault, and Moinas (2015), Budish, Cramton, and Shim (2015), and Menkveld and Zoican (2015).

We also divide all traders into four groups based on their inventory for each stock-day: LARGE, MEDIUM, SMALL, and NOTRADE. Inventory at the end of the day is calculated by the absolute value of the number of shares bought minus the number of shares sold during the day, as a fraction of the total volume of shares bought and sold in the day for that particular stock. In particular, if a trader's inventory is equal to $100 \%$, we consider the trader to be a LARGE inventory trader. If a trader's inventory is not computable, we consider the trader to be a NOTRADE agent. The rest of the distribution is split on a stock-day basis relative to the median to form the MEDIUM and SMALL inventory groups. It is important to note that we differentiate between a trader who ends a particular day with a flat inventory as a result of buy and sell activity throughout the day, and a NOTRADE agent. It should also be noted that NOTRADE agents include traders that submit orders, but whose orders are not filled. Table II briefly summarizes our scheme.

\section{[Place Table II about here]}

\section{B. HFT identification: summary statistics}

Based on the classification of the groups we report in Table II, we calculate several summary statistics on the behavior of the different groups of traders as reported in Table III.

\section{[Place Table III about here]}

Table III presents the summary statistics for speed and inventory for each group under our classification procedure. The median minimum speed in the FAST group varies, across 
different inventory subgroups, from nine to four milliseconds. The MODERATE group exhibits a much higher median minimum speed, ranging from 3.3 to 6.8 seconds. The SLOW group has a median minimum speed of above 300 seconds. Note that similar patterns hold if we focus on the bottom $1 \%, 5 \%$, or $10 \%$ of the speed distribution, instead of the specified minimum speed.

By construction, the LARGE inventory subgroup always has a 100\% end-of-day inventory, meaning that, during the day, traders either only buy or only sell the stock. Traders from the MEDIUM inventory subgroup tend to end their trading day with an inventory of around $65 \%$ of the total volume traded, while traders from the SMALL inventory subgroup can end up with an inventory as low as 15\%. Patterns for the median intraday inventory and its volatility are consistent with the end-of-day inventory. Besides that, we also estimate the number of times during the day that the signed inventory crosses zero. FAST/SMALL and MODERATE/SMALL traders exhibit the largest median number of zero crossings. Based on the speed and inventory classifications, one can consider FAST/SMALL traders as HFTs, as indicated in brackets in all the tables.

We emphasize that we use information from the continuous period on the same stock-day to describe trader behavior in the pre-opening period. This is motivated by changes in the traders' strategies from one day to another as documented by Table IV for the transition frequency matrix of trader strategies. In particular, we observe that on average, only in $27.84 \%$ of cases do traders remain in the same group from one active stock-day to the next. The most persistent group is the SLOW/LARGE group (51.92\%). Among FAST traders, the greatest persistence is observed for the FAST/SMALL (HFTs) group (40.99\%). Within the same speed group, ignoring the differences in inventory, we observe more persistence: on average, traders tend to remain in the same speed group in $61.40 \%$ of the cases. Traders tend to remain in the same inventory group in $44.45 \%$ of the cases, on average, ignoring the speed dimension, with the largest contribution to this persistence coming from the LARGE inventory group.

\section{[Place Table IV about here]}

As far as we know, all the empirical studies in the literature, except ASIC (2013) and 
Benos and Sagade (2016), assume that HFTs behave in an identical manner every day, and for every stock. We do not claim that one approach dominates the other, but simply that they have different purposes. Our objective is to identify the impact on market quality of HFT activity when these traders use their speed capacity.

To gain an idea of the difference in the traders' characteristics, if we use one of the classification procedures proposed in the literature, we apply a scheme following Brogaard, Hagströmer, Norden, and Riordan (2015), a modification of the Kirilenko, Kyle, Samadi, and Tuzun (2016) approach, which splits traders into two groups, namely HFTs and non-HFTs, based on three criteria: end-of-day inventory, inventory at the end of each minute, and volume traded. Our analysis show that this classification does not always identify very fast traders and their activity during the pre-opening period. In fact, based on this classification scheme, we identify only five traders as HFTs with very little activity coming from them in the preopening period. Note that most of the observations are marked as non-HFTs, suggesting that the Kirilenko, Kyle, Samadi, and Tuzun (2016) scheme is a stricter (narrower) classification of HFTs than the classification we propose. We believe that the diversity of market participants in the TSE better suits our more comprehensive approach than the narrower alternative scheme proposed earlier. We, therefore, prefer our HFT identification strategy, given that the Kirilenko, Kyle, Samadi, and Tuzun (2016) scheme seems less appropriate for the TSE market, at least with the current thresholds in place. ${ }^{22}$

\section{Results}

We now present the results of our empirical investigation, in line with the hypotheses presented in Section II above. The presentation consists of three subsections, dealing with (1) the participation of HFTs at three different times, the pre-opening period, the opening auction, and the continuous trading period; (2) the contribution of HFTs to price discovery during the pre-opening and continuous trading periods; and (3) the impact of HFT trading on liquidity in the opening call auction and the continuous trading period. In the robustness analysis (in Section VI), we also check whether HFTs change their behavior in terms of (1),

\footnotetext{
${ }^{22} \mathrm{~A}$ detailed description of the results is reported in the Internet Appendix D.
} 
(2), and (3) in different volatility regimes.

\section{A. HFT participation}

HYPOTHESIS 1: HFTs are the most active participants in the pre-opening, opening call auction and continuous trading periods.

We investigate the participation of the different trader groups, and specifically those that we identify as HFTs (the FAST/SMALL group), in the pre-opening period, the opening auction, and the first 30 minutes of the continuous period, using the whole continuous period as a benchmark. We first compute the presence ratio $(P R)$ defined in equation (1). Table V shows the $P R$ s of all 12 groups in the three periods.

\section{[Place Table V about here]}

The $P R$ s of HFTs during the pre-opening period and the opening call auction are $26.96 \%$ and $17.47 \%$, respectively. These results address the question of whether HFTs participate in the pre-opening period, despite the fact that no trading occurs at that point in time. Our analysis shows that, even though there is no trading, one quarter of HFTs do participate in the pre-opening period. ${ }^{23}$ Table $\mathrm{V}$ also shows that in the continuous period (the first 30 minutes), 83.65\% are present, confirming that the large majority of HFT traders do participate during the early part of the trading day. Table $\mathrm{V}$ also shows that $16.16 \%$ of HFTs actively participate in all three periods: pre-opening, opening auction, and continuous periods. The table also reports a distinction between HFTs that actively trade in the opening auction (Active-w-Trade) along with those that are present in the pre-opening period but do not trade during the opening auction (Active-w/o-Trade) and, on the other hand, those that are Non-Active in both the pre-opening period and the opening auction, but do participate in the continuous period. In the continuous period, almost all of the Active-w-Trade HFTs also participate in the continuous period, and the same applies to Active-w/o-Trade HFTs. However, the majority of HFTs present in the continuous period are Non-Active (58.91\%). Figure 3 shows the presence of HFT traders during different periods of the trading day. In

\footnotetext{
${ }^{23}$ For a detailed description of the order flow by the different groups of traders during the pre-opening period, see the Internet Appendix E.
} 
particular, we show that $26.96 \%$ of HFTs are present in the pre-opening period. Out of these traders, $65 \%(=17.47 / 26.96)$ execute their orders in the opening call auction. Among those traders that execute their orders in the opening call auction, $93 \%(=16.16 / 17.47)$ are also present in the first 30 minutes of the continuous trading period.

\section{[Place Figure 3 about here]}

Going beyond mere presence, we next compute the level of activity, based on quotes submitted by traders in the different periods, that is, the $Q A R$ defined in equation (2). In Table VI, Panel A, we report the average $Q A R$.

\section{[Place Table VI about here]}

Table VI, Panel A, shows that HFTs represent the trader group with the largest proportion of orders submitted, with about $20.58 \%$ of the total orders submitted during the pre-opening period, defined as averages, which splits into about $4.98 \%$ by the Active-w/oTrade and $15.60 \%$ by the Active-w-Trade group, respectively. For comparison, the table shows that HFTs are also the trader group submitting the majority of quotes during the continuous period (42.05\%), almost double the percentage submitted during the pre-opening period. This indicates that the majority of the quotes in the continuous period are submitted by HFTs that are not present during the pre-opening period (Non-Active). We also analyze the behavior of traders based on aggressive orders alone. We find that in the pre-opening period, HFTs places the highest number of aggressive orders in relation to the total number of orders, which indicates their interest in affecting the price. ${ }^{24}$ The variation of the $Q A R$ within the speed and inventory categories also suggests that both the speed and inventory dimensions are important when analyzing the impacts of the different trader groups on market quality.

In Table VI, Panel B, we perform a formal test of whether this activity is also statistically significant (see equation (13)). We estimate the coefficient using the Active-wTrade SLOW/SMALL group as a base case. We observe that HFTs are confirmed as being statistically significantly present, with the largest coefficient among all groups during the

\footnotetext{
${ }^{24}$ Please see Internet Appendix F for details.
} 
pre-opening period and the first 30 minutes of the continuous period. The test of significance is based on clustered standard errors (by stock). We also computed robust standard errors and the results were similar. We also performed a test of whether Active-w-Trade HFTs have a statistically larger presence during the pre-opening period than Active-w-Trade FAST/MEDIUM, MODERATE/SMALL, and MODERATE/MEDIUM traders. We reject the null hypothesis that the coefficients are the same at the $1 \%$ level, for Active-w-Trade MODERATE/MEDIUM traders. However, we cannot reject the hypothesis that quoting activity is the same for FAST/SMALL (HFTs), FAST/MEDIUM, and MODERATE/SMALL traders. Put differently, Active-w-Trade HFTs are among the top three trader groups in terms of quoting activity during the pre-opening period, while Non-Active HFTs are the most active group during the continuous period.

Finally, we investigate whether HFTs as a trader group have a $Q A R$ that is larger than non-HFTs. The results are reported in Table VI, Panel C, and show that HFTs have a quoting activity ratio that is $6.11 \%$ larger than that of non-HFTs in the pre-opening period (12.09\% larger in the continuous period). Active-w-Trade HFTs have a quoting activity ratio that is $11.39 \%$ larger than all other traders, as a group. Non-Active HFTs have a quoting activity ratio that is $28.10 \%$ larger than all the other traders in the continuous period. This test indicates that, even if there is a huge dispersion in their participation across stocks and across days, there is always a significant fraction of quotes posted by HFTs in both the pre-opening and continuous periods.

So far, we have presented a comparative analysis of the pre-opening and continuous periods. We repeat the above analysis for participation in the opening auction, using trades rather than quotes, to measure the trading activity ratio $T A R$, defined in equation (3). The results are reported in Table VII.

\section{[Place Table VII about here]}

Table VII, Panel A, shows that HFTs do participate significantly in the opening auction: $36.65 \%$ of the volume traded during the auction relates to HFTs, either as sellers or buyers. Compared with the other trader groups, they trade the second largest fraction. In line with previous empirical evidence, HFTs also contribute greatly to the volume traded during the 
continuous period. The same panel shows a large difference between the volume traded by HFTs present in the auction, and those present only during the continuous period. Those that are not active during the auction (Non-Active) are responsible for $44.40 \%$ of the total volume of trades during the continuous period, while those that are active during the opening auction (Active-w-Trade) account, on average, for $19.79 \%$.

We next perform a test of whether HFT participation is statistically different from zero, and not driven by outliers (see equation (13)). These results are reported in Table VII, Panel B. As shown, the presence of HFTs in all periods is significantly different from the $T A R$ of the base-case group (Active-w-Trade SLOW/SMALL), and also different from zero, at the $1 \%$ level. HFTs are the second among the trader groups participating in the opening auction, in terms of $T A R$, while the most active group are the FAST/MEDIUM traders (the $F$-test we perform shows the difference between the two groups' trading activity to be statistically different from zero).

We also perform a general comparison of HFTs versus non-HFTs, by grouping all the other trader groups into one, and the results are reported in Table VII, Panel C, showing that, during the opening call auction, HFTs exhibit a level of trading activity that is $16.23 \%$ higher than the level of the other traders, and showing this difference in presence to be statistically different from zero. During the continuous period, HFTs have a trading activity level that is $17.50 \%$ larger than that of the other traders. However, Non-Active (Active-wTrade) HFTs have a trading activity level $38.42 \%$ (12.86\%) larger than that of the other traders.

\section{B. Price discovery}

HYPOTHESIS 2: Of all types of trader, HFTs contribute the most to price discovery in the pre-opening and continuous trading periods.

Our data allow us to measure the $P D C$ by individual order and, in particular, by individual aggressive order, so that we can aggregate the WPC according to the trader group that submitted the order, to show the proportion of the price contribution made by a particular trader group. We calculate the $W P C$ as described in equation (7). The results of the $W P C$ 
analysis are reported in Table VIII.

\section{[Place Table VIII about here]}

Table VIII, Panel A, shows that the main contribution to the $W P C$ comes from FAST/MEDIUM $(-26.66 \%)$ traders, followed by the HFTs (-16.23\%) and MODERATE/SMALL (-13.61\%) traders, during the pre-opening period. Moreover, we find that HFTs that are Active-wTrade during the auction contribute $13.67 \%$ to the $W P C$, while those that are Active-w/oTrade account for only $2.56 \%$. The panel also shows the results of the $W P C$ analysis for the first 30 minutes of the continuous trading period. HFTs, in this case, contribute $35.47 \%$ to price discovery, the largest contribution among all the trader groups. However, there is a lot of heterogeneity among HFTs, with big differences between those that actively participate in the pre-opening period and the opening auction, and the Non-Active HFTs. Non-Active HFTs are responsible for $-37.99 \%$ of the $W P C$ during the continuous period. On the contrary, among the traders that are active during pre-opening period, Active-w-Trade HFTs no longer contribute to price discovery, and if anything detract from it $(+3.85 \%)$.

Table VIII, Panel B, presents the results of a formal test of whether the price discovery caused by HFTs is statistically significant. We estimate the regression using the Activew-Trade SLOW/SMALL group as the base case. The largest price discovery contribution during the pre-opening period is made by the Active-w-Trade FAST/MEDIUM traders, followed by the Active-w-Trade HFTs. In the continuous period, the Non-Active HFTs show the largest $W P C$, while the Active-w-Trade HFTs show $W P C$ that are not statistically different from zero. Therefore, the statistical analysis indicates that Active-w-Trade HFTs do not contribute to price discovery, but neither do they harm it.

Table VIII, Panel C, investigates whether HFTs as a trader group have a WPC larger than that of non-HFTs. It shows that HFTs as a group contribute more to price discovery than the other trader groups (-3.70\%, which is statistically significant at the $1 \%$ level), in the

pre-opening period. However, only Active-w-Trade HFTs show a significant amount of price discovery that is statistically larger than that made by non-HFTs during the pre-opening period. Active-w/o-Trade HFTs actually reduce price discovery during the pre-opening period. In the continuous period, HFTs contribute significantly to price discovery, with this 
contribution coming largely from Non-Active HFTs. Active-w-Trade HFTs have an impact that is not statistically significant, while Active-w/o-Trade HFTs deteriorate price discovery. These results confirm the heterogeneity of HFTs and the difference in their contributions to market quality, conditional on the period considered: pre-opening versus continuous. ${ }^{25}$

One of the characteristics of the opening price is that its volatility is larger than that of the closing price (Stoll and Whaley (1990); Amihud and Mendelson (1991); Berkman, Koch, Tuttle, and Zhang (2012)). This stylized fact has been attributed to many factors, including the accumulation of information and concentration of orders overnight, with attendant consequences for asymmetry of information, and market-maker intervention. Amihud and Mendelson (1991) conclude that the higher volatility of the opening price of the morning session is due to the staleness of the reference price, which is the closing price of the previous day (18 hours prior to opening). However, in this section, the WPC analysis for the preopening period is based on the auction price, which may be less stale. To check whether this assumption affects the overall results for the major contributors to price discovery during the pre-opening period, we consider the average price between 9:30 and 9:35 a.m. as a reference price, rather than the auction price, which is used for investigating price discovery for the continuous period. Our analysis confirms that the selection of the reference price does not affect our conclusions. The results are reported in Table IA.6 of Internet Appendix G. We also investigate the impact of a particular order type on price discovery (similarly to Barclay and Warner (1993) and Chakravarty (2001)). Breaking down the WPC order by order and trader by trader, we find that the types of order contributing most to the $W P C$ are new limit and market orders submitted by Active-w-Trade traders. Cancellations of market orders and price revisions of limit orders also contribute to price discovery. On the other hand, quantity revisions and cancellations of limit orders increase the deviation of the quoted price from the opening price. ${ }^{26}$

In the literature there have been other approaches used to investigate price discovery. In the robustness section (see Section VI), we summarize the results of several other comple-

\footnotetext{
${ }^{25}$ The results of the test of whether the contributions from trader groups are significantly different across stock-date are very similar to Table VIII, Panel B (the results are reported in Table IA.5 of Internet Appendix $\mathrm{G})$.

${ }^{26}$ For a detailed description of the analysis see the Internet Appendix G, Table IA.7.
} 
mentary analyses that we performed related to price discovery. First, we investigate price discovery by looking at the deviation of the pre-opening quoted price from the opening price, computed for each second of the entire pre-opening period, in different stock groups. Second, we test the price efficiency of the pre-opening quotes, using an unbiasedness regression that has been widely used in the literature (e.g., Biais, Hillion, and Spatt (1999), Barclay and Hendershott (2003), and Barclay and Hendershott (2008)). This analysis highlight results that are consistent with the $W P C$ study presented in this section.

\section{Liquidity provision}

HYPOTHESIS 3: Of all types of trader, HFTs contribute the most to liquidity in the opening call auction and the continuous trading period.

In this subsection, we investigate the contribution of HFTs to liquidity provision in the opening auction and the continuous period. We use the Net Liquidity Provision measure $(N L P)$ defined in equation (10) based on the liquidity-demanding and liquidity-supplying trading volumes, relative to the total trading volume during the opening auction, and the first 30 minutes of the continuous period. Table IX presents the NLPs during the opening auction and the continuous period.

\section{[Place Table IX about here]}

Table IX, Panel A, shows that HFTs provide liquidity to the market during the opening auction, but consume liquidity during the continuous period. Again, we find considerable heterogeneity among HFTs: Active-w-Trade HFTs provide liquidity during both the opening auction and the continuous period, while Active-w/o-Trade HFTs contribute to liquidity provision during the continuous period. However, Non-Active HFTs largely consume liquidity. This result is similar to what we find for the continuous period in relation to price discovery. The result can be theoretically justified if we keep in mind the canonical microstructure models of Kyle (1985), Glosten and Milgrom (1985) and others, in which informed investors consume liquidity. Indeed, in the Kyle model, uninformed liquidity traders supply liquidity, on average, by selling/buying for other reasons, such as liquidity needs, while informed in- 
vestors take advantage of this liquidity provision by executing their informed trades against them.

In Table IX, Panel B, we perform the regression analysis (see equation (13)) and the results confirm those reported in Panel A. Non-Active HFTs significantly consume liquidity and the other HFTs contribute to liquidity in both the opening auction and the continuous period. We perform an $F$-test to compare liquidity provision in the opening call auction by FAST/SMALL (HFTs), MODERATE/SMALL, and MODERATE/MEDIUM traders. We show that the main liquidity provider in the opening call auction is the MODERATE/MEDIUM trader group, while HFTs share second place with the MODERATE/SMALL traders. In Table IX, Panel C, we compare HFTs to non-HFTs and find that in the pre-opening period they contribute significantly to liquidity, while in the continuous trading period they largely consume liquidity. This result, however, is driven completely by Non-Active HFTs.

To conclude, Active-w-Trade HFTs are among the main liquidity providers in the opening call auction and the continuous period. Non-Active HFTs are major liquidity demanders in the continuous period.

\section{Robustness checks}

We present in this section several robustness checks related to HFT behavior in different volatility regimes and investigate the contribution to price discovery by HFTs using two other approaches used in the literature. The first approach investigates the contribution of HFTs to the speed of price discovery. The second approach is based on the unbiasedness of the pre-opening quotes, another measure the literature proposes for investigating price discovery.

\section{A. HFT behavior in different volatility regimes}

In this robustness check, we analyze whether the above results are affected by marketwide (systematic) and stock-specific (idiosyncratic) movements in stock prices from one day to the next. In fact, the SEC (2010) and others express concern about the impact of HFTs 
on market quality, especially during times of stress. In our analysis, we distinguish between systematic and idiosyncratic volatility. In this spirit, we investigate whether we can observe differences in the participation, price discovery, and liquidity provision of HFTs, when the daily price dispersion of the market is large, and when the daily price dispersion of a single stock is large, even when market volatility is low. We measure market volatility by the difference between the daily highest value of the index TOPIX and the lowest value, and we measure stock volatility by the difference between the daily highest price of the stock and the lowest price. We then calculate the median during the sample period of the aforementioned difference for the market and stock volatilities. We set a systematic volatility dummy to one, when the value of the market volatility is above the median, and zero otherwise. We set the stock volatility dummy to one, when the value of the stock volatility is above the median, and zero otherwise. We estimate the idiosyncratic volatility dummy as the difference between the stock volatility dummy and the product of the systematic volatility dummy and the stock volatility dummy. ${ }^{27}$

By interacting the systematic and idiosyncratic volatility dummies with the trader group dummies, we test the hypothesis of whether there is any difference in HFT behavior, during the days when there is high systematic or idiosyncratic volatility:

$$
\begin{aligned}
Y_{p, j, k, l}= & a_{0, p}+\sum\left(a_{l, p} * I_{l}+b_{l, p} * I_{l} * \text { SysVol }_{k}+c_{l, p} * I_{l} * \operatorname{IdioVol}_{j, k}\right) \\
& +b_{0, p} \text { SysVol }_{k}+c_{0, p} \text { IdioVol }_{j, k}+e_{p, j, k, l}
\end{aligned}
$$

where $Y_{p, j, k, l}$ is the investigated measure, that is, participation measured by $Q A R$ and $T A R$, price discovery by $W P C$, and liquidity provision by NLP. $p$ is the period considered (preopening, opening auction, continuous) for stock $j$, day $k$, and trader group $l . I_{l}$ is a dummy variable that equals one for the trader group $l$. SysVol $k$ is a dummy variable that equals one, if the high minus low range for the TOPIX index on day $k$ is higher than the median. $\mathrm{IdioVol}_{k}$ is a dummy variable that equals one, if the high minus low range for stock $j$ on day $k$ is higher than the median and $S y s V_{k}$ is not equal to one on day $k$. We then test whether

\footnotetext{
${ }^{27}$ We acknowledge that using the median may not be enough to identify stressful periods. However, since we only have 41 days in our sample, tests based on stricter criteria may suffer from a lack of power, as happens with the test in Brogaard, Hendershott, and Riordan (2014). Nevertheless, in unreported results, we repeat the analysis using the 90th-percentile instead of the median and, if anything, the results become stronger.
} 
the coefficients $b_{l}$ and $c_{l}$ are statistically different from zero. In Tables X, XI, XII, and XIII, we report $b_{l}$ (Panel A) and $c_{l}$ (Panel B) from the regressions for the quoting activity, trading activity, price discovery, and liquidity provision of traders in different volatility regimes.

\section{[Place Table X about here]}

The results in Table X confirm that all HFTs, except Non-Active HFTs, increase their quoting activity relative to other trader groups, in times of high systematic and idiosyncratic volatility, with this effect being more pronounced for idiosyncratic volatility. Non-Active HFTs do not change their behavior.

The results of the trading activity analysis are reported in Table XI. The analysis also shows that the trading activity during the opening auction and the continuous trading period of Active-w-Trade HFTs increases with systematic volatility, while other HFTs do not change their behavior. Idiosyncratic volatility also leads to an increase in the trading activity of HFTs relative to other trader groups, with the exception of Non-Active HFTs, who do not change their behavior.

\section{[Place Table XI about here]}

This is consistent with the analysis of the QAR: Non-Active HFTs tend to behave in a similar manner when volatility is higher. This finding is consistent with the idea that idiosyncratic volatility is indicative of the larger amount of stock-specific information, rather than stress alone. These results confirm the results of Brogaard, Hendershott, and Riordan (2014) for the continuous period. However, by considering both the systematic volatility and the idiosyncratic volatility, our analysis shows that, in both the pre-opening and the continuous section, HFTs do not reduce their participation on days when systematic or idiosyncratic volatility is high.

We then investigate whether $W P C$ is affected by different systematic and idiosyncratic volatility regimes. We find that most of the HFTs do not change their contribution to price discovery across different volatility regimes, with only two exceptions: First, Non-Active HFTs contribute more to price discovery during the first 30 minutes of the continuous trading period when systematic volatility is high. Second, Active-w-Trade HFTs contribute more 
to price discovery during the pre-opening period in times of high idiosyncratic volatility. Remarkably, none of the HFTs decrease their price discovery contribution in regimes with high systematic/idiosyncratic volatility.

\section{[Place Table XII about here]}

We next investigate whether there are differences in HFTs' liquidity provision across different volatility regimes. We observe that HFTs' liquidity provision in the opening call auction is not affected by volatility. We show that, in times of high systematic/idiosyncratic volatility, Non-Active HFTs consume less liquidity than in times of low systematic/idiosyncratic volatility. Active-w/o-Trade HFTs also increase their liquidity provision in times of high idiosyncratic volatility. The results are reported in Table XIII. In brief, HFTs' liquidity provision does not decrease in highly volatile periods.

\section{[Place Table XIII about here]}

To sum up, we do not find any evidence that HFTs decrease their presence in the market, or stop facilitating price discovery or providing liquidity, in times of high systematic or idiosyncratic volatility.

\section{B. HFT and Speed in price discovery}

One of the questions we aim to answer with this paper concerns price discovery. We have considered the pre-opening period without distinguishing the earlier part of the period from the minute just before the auction. However, the order submission activity during the pre-opening section varies as the auction time approaches. As further analysis of the role of HFTs on price discovery, we aim to investigate how HFT affect the speed of the price convergence to the auction price. We perform this analysis by studying whether stocks with a greater presence of HFTs show different patterns of price convergence of the quoted price to the opening price. We looked at the distribution of HFTs activity across stocks and observed that there is a wide variation in their activity from stock to stock during the pre-opening period. By splitting the sample of stocks into two groups: stocks where the activity of HFTs is large compared with all the other stocks, our analysis shows that the deviation from the 
opening price is always smaller for the stocks where there is an active presence of HFTs i.e., their presence improves the speed of convergence to the auction price and, therefore, the efficiency of the price discovery process. ${ }^{28}$

\section{Unbiasedness of the quoted price}

The literature on price discovery in the pre-opening period investigates price efficiency by analyzing when the quoted price is statistically equal to the opening price during the pre-opening period. ${ }^{29}$ The earlier the two prices are statistically equal, the greater is the price efficiency. We perform the test for the price efficiency of the pre-opening quotes by distinguishing stocks where HFT are largely active from other stocks.

Our analysis shows that the quoted price for stocks with a greater presence of HFTs is statistically equal to the opening price almost immediately after the first 10 minutes. In contrast, for stocks where the HFTs presence is lower, this difference becomes insignificant only few seconds before the opening call auction. This indicates that price efficiency is higher for the stocks with higher HFT participation (see the Internet Appendix H for a detailed description of the analysis and the results).

\section{Conclusion}

The market pre-opening period and the opening call auction are important features of many stock markets nowadays. Our study focuses on HFT activity in these trading periods, which has come to dominate global equity markets. Key questions we ask in this paper are whether, in the absence of trading, very fast traders (including HFTs) still participate in the market, and how the presence of fast traders contributes to price discovery in the preopening period, and to liquidity provision in the opening call auction. In order to empirically investigate these questions, we use a unique dataset provided by the TSE, which allows us to develop a more comprehensive classification of traders than in the prior literature and

\footnotetext{
${ }^{28}$ For a detailed description of this analysis see the Internet appendix G.

${ }^{29}$ Among other papers that use an unbiasedness regression to investigate price discovery are Biais, Hillion, and Spatt (1999), Barclay and Hendershott (2003, 2008), Comerton-Forde and Rydge (2006), and Chakrabarty, Corwin, and Panayides (2011).
} 
to investigate the behavior of different categories of traders, based on their capability for high-speed trading.

We classify traders into three speed and four inventory groups (making a total of 12 groups) on a stock-day basis. We observe that, on average, in only $28 \%$ of cases do traders remain in the same speed/inventory group from one day to the next. It is, therefore, not appropriate to assume that HFTs always trade all stocks in the same manner, every day. Hence, our classification of traders based on both speed of trading and inventory, vary across stocks and across days to focus on the effect of HFT activity on market quality when HFTs actually use their speed capacity.

Our empirical results for the TSE show that HFTs participate in the pre-opening period and in the opening auction to a lesser extent than in the continuous trading period. In particular, only a quarter of HFTs participate during pre-opening period. However, HFTs exhibit the largest quoting activity during pre-opening period and the second largest trading activity at the opening call auction among all 12 trader groups.

We find that HFTs orders play a central role in price discovery during pre-opening period and in liquidity provision at the opening call auction. We also show that there is a large heterogeneity in the contribution to market quality by HFTs. As a group, HFTs provide liquidity in the call auction but consume liquidity in the continuous period. However, HFTs that execute their orders during the opening call auction (Active-w-Trade) provide liquidity both in the call auction and the continuous trading period. On the contrary, HFTs that do not participate in the pre-opening period (Non-Active) are the main liquidity consumers, while playing a greater role in price discovery during the continuous trading period.

To conclude, our results suggest that HFTs that participate in the pre-opening period are different from those that only participate in the continuous trading period and play a different role in price formation and liquidity provision in pre-opening, opening call and continuous section. Future studies should take into consideration this heterogeneity when they investigate the role of HFTs on market quality. 


\section{References}

Ait-Sahalia, Yacine, and Mehmet Saglam, 2014, High frequency traders: Taking advantage of speed, Working paper.

Amihud, Yakov, and Haim Mendelson, 1991, Volatility, efficiency, and trading: Evidence from the Japanese stock market, Journal of Finance 46, 1765-1789.

ASIC, 2013, Dark liquidity and high-frequency trading, Australian Securities and Investments Commission: Report 331.

Barclay, Michael J, and Terrence Hendershott, 2003, Price discovery and trading after hours, Review of Financial Studies 16, 1041-1073.

Barclay, Michael J, and Terrence Hendershott, 2008, A comparison of trading and nontrading mechanisms for price discovery, Journal of Empirical Finance 15, 839-849.

Barclay, Michael J, and Jerold B Warner, 1993, Stealth trading and volatility: Which trades move prices?, Journal of Financial Economics 34, 281-305.

Baron, Matthew, Jonathan Brogaard, Björn Hagströmer, and Andrei Kirilenko, 2016, Risk and return in high-frequency trading, Available at SSRN.

Benos, Evangelos, and Satchit Sagade, 2016, Price discovery and the cross-section of highfrequency trading, Journal of Financial Market Forthcoming.

Berkman, Henk, Paul D Koch, Laura Tuttle, and Ying Jenny Zhang, 2012, Paying attention: overnight returns and the hidden cost of buying at the open, Journal of Financial and Quantitative Analysis 47, 715-741.

Biais, Bruno, and Thierry Foucault, 2014, HFT and market quality, Bankers, Markets and Investors 128, 5-19.

Biais, Bruno, Thierry Foucault, and Sophie Moinas, 2015, Equilibrium fast trading, Journal of Financial Economics 116, 292-313.

Biais, Bruno, Pierre Hillion, and Chester Spatt, 1995, An empirical analysis of the limit order book and the order flow in the Paris Bourse, Journal of Finance 50, 1655-1689.

Biais, Bruno, Pierre Hillion, and Chester Spatt, 1999, Price discovery and learning during the preopening period in the Paris Bourse, Journal of Political Economy 107, 1218-1248. 
Boehmer, Ekkehart, Kingsley YL Fong, and Juan Julie Wu, 2014, International evidence on algorithmic trading, Working paper.

Brogaard, Jonathan, 2010, High frequency trading and its impact on market quality, Working paper.

Brogaard, Jonathan, Björn Hagströmer, Lars L Norden, and Ryan Riordan, 2015, Trading fast and slow: Colocation and market quality, Review of Financial Studies 28, 3407-3443.

Brogaard, Jonathan, Terrence Hendershott, and Ryan Riordan, 2014, High-frequency trading and price discovery, Review of Financial Studies 27, 2267-2306.

Budish, Eric B., Peter Cramton, and John J. Shim, 2015, The high-frequency trading arms race: Frequent batch auctions as a market design response, Quarterly Journal of Economics 130, 1547-1621.

Cao, Charles, Eric Ghysels, and Frank Hatheway, 2000, Price discovery without trading: Evidence from the NASDAQ preopening, The Journal of Finance 55, 1339-1365.

Cespa, Giovanni, and Thierry Foucault, 2011, Learning from prices, liquidity spillovers, and market segmentation, CEPR Discussion Paper No. DP8350.

Chaboud, Alain, Benjamin Chiquoine, Erik Hjalmarsson, and Clara Vega, 2014, Rise of the machines: Algorithmic trading in the foreign exchange market, The Journal of Finance 5, 2045-2084.

Chakrabarty, Bidisha, Shane A Corwin, and Marios A Panayides, 2011, When a halt is not a halt: An analysis of off-NYSE trading during NYSE market closures, Journal of Financial Intermediation 20, 361-386.

Chakravarty, Sugato, 2001, Stealth-trading: Which traders' trades move stock prices?, Journal of Financial Economics 61, 289-307.

Chi-X, 2013, CHI-X Japan Monthly Statistics Report.

Ciccotello, Conrad S, and Frank M Hatheway, 2000, Indicating ahead: Best execution and the NASDAQ preopening, Journal of Financial Intermediation 9, 184-212.

Comerton-Forde, Carole, and James Rydge, 2006, The influence of call auction algorithm rules on market efficiency, Journal of Financial Markets 9, 199-222.

Duong, Huu Nhan, Petko S Kalev, and Chandrasekhar Krishnamurti, 2009, Order aggressiveness of institutional and individual investors, Pacific-Basin Finance Journal 17, 533-546. 
Easley, David, Marcos M López de Prado, and Maureen O’Hara, 2012, Flow toxicity and liquidity in a high-frequency world, Review of Financial Studies 25, 1457-1493.

Foucault, Thierry, Johan Hombert, and Ioanid Roşu, 2016, News trading and speed, Journal of Finance 71, 335-382.

Glosten, Lawrence R, and Paul R. Milgrom, 1985, Bid, ask and transaction prices in a specialist market with heterogeneously informed traders, Journal of Financial Economics $1,71-100$.

Gomber, Peter, Björn Arndt, Marco Lutat, and Tim Uhle, 2011, High-frequency trading, Working paper.

Hagströmer, Björn, and Lars Norden, 2013, The diversity of high-frequency traders, Journal of Financial Markets 16, 741-770.

Hasbrouck, Joel, 1995, One security, many markets: determining the contributions to price discovery, Journal of Finance 50, 1175-1199.

Hasbrouck, Joel, 2002, Stalking the "efficient price" in market microstructure specifications: an overview, Journal of Financial Markets 5, 1-19.

Hasbrouck, Joel, and Gideon Saar, 2013, Low-latency trading, Journal of Financial Markets $16,646-649$.

Hendershott, Terrence, Charles M Jones, and Albert J Menkveld, 2011, Does algorithmic trading improve liquidity?, Journal of Finance 66, 1-33.

Hendershott, Terrence, and Pamela C Moulton, 2011, Automation, speed, and stock market quality: The NYSE's hybrid, Journal of Financial Markets 14, 568-604.

Hendershott, Terrence, and Ryan Riordan, 2013, Algorithmic trading and the market for liquidity, Journal of Financial and Quantitative Analysis 48, 1001-1024.

Hosaka, G., 2014, Analysis of high-frequency trading at Tokyo Stock Exchange, Working paper.

Japannext, SBI, 2013, Japannext PTS Monthly Statistics.

Jones, Charles M, 2013, What do we know about high-frequency trading?, Working paper.

Jovanovic, B, and Albert J Menkveld, 2015, Middlemen in limit-order markets, Working paper. 
Kirilenko, Andrei A, Albert S Kyle, Mehrdad Samadi, and Tugkan Tuzun, 2016, The Flash crash: High frequency trading in an electronic market, Journal of Finance, Forthcoming.

Korajczyk, Robert A., and Dermot Murphy, 2015, High frequency market making to large institutional trades, Available at SSRN.

Kyle, A S, 1985, Continuous auctions and insider trading, Econometrica 53, 1315-1335.

Lewis, Michael, 2014, Flash boys: A Wall Street revolt (WW Norton \& Company).

Madhavan, Ananth, and Venkatesh Panchapagesan, 2000, Price discovery in auction markets: A look inside the black box, Review of Financial Studies 13, 627-658.

Malinova, Katya, Andreas Park, and Ryan Riordan, 2013, Do retail traders suffer from high frequency traders?, Working paper.

Menkveld, Albert J, 2013, High frequency trading and the new market makers, Journal of Financial Markets 16, 712-740.

Menkveld, Albert J, and Marius A Zoican, 2015, Need for speed? Low latency trading and adverse selection, Working paper.

Pagnotta, Emiliano, and Thomas Philippon, 2011, Competing on speed, Working paper.

Raman, V., and P. Yadav, 2014, Liquidity provision, information, and inventory management in limit order markets: An analysis of order revisions, Working paper.

Ranaldo, Angelo, 2004, Order aggressiveness in limit order book markets, Journal of Financial Markets 7, 53-74.

SEC, 2010, Concept release on equity market structure, Release No. 34-61358, File No. S7-02-10.

SEC, 2014, Equity market structure literature review. Part II: High frequency trading.

Stoll, Hans R, and Robert E Whaley, 1990, Stock market structure and volatility, Review of Financial Studies 3, 37-71.

TSE, 2015, Tokyo Stock Exchange: Guide to TSE trading methodology.

Van Bommel, Jos, 2011, Measuring price discovery: the variance ratio, the $\mathrm{r} 2$, and the weighted price contribution, Finance Research Letters 3, 112-119.

Yamamoto, Ryuichi, 2011, Order aggressiveness, pre-trade transparency, and long memory in an order-driven market, Journal of Economic Dynamics and Control 35, 1938-1963. 


\section{Table I Traders' characteristics during the continuous trading period}

This table shows characteristics of the trading infrastructure and behavior of traders on the Tokyo Stock Exchange, where 5,580 unique virtual server IDs are used by traders. We trace the usage of individual virtual servers and, during the continuous trading period, identify 3,048 trading desks (traders) using single (or multiple) virtual server(s) for their trading. All traders are sorted into one of the six groups based on the number of VSs they utilize. For each group, we describe the number of traders, median number of virtual servers used per trade, number of stocks traded (in total and per virtual server), median speed (minimum time elapsed between two consecutive orders for the same stock), median inventory (the median of the endof-the-day inventory), median number of quotes, and median volume share per day (the proportion of the buy volume plus the sell volume per trading desk). These characteristics are based on the continuous trading period activity for the period of April and May 2013, for 1,702 stocks on the Tokyo Stock Exchange. Order flow data, with order IDs as well as virtual server IDs, are provided by the Tokyo Stock Exchange.

Grouped by number of virtual servers used

\begin{tabular}{lcccccc}
\hline & 1 & $2-9$ & $10-19$ & $20-29$ & $30-39$ & $40-41$ \\
\hline \# of traders & 2,718 & 215 & 80 & 19 & 11 & 5 \\
\# of virtual servers & 1 & 3 & 12.5 & 21 & 32 & 40 \\
\# of stocks traded in total & 183 & 247 & 363 & 461 & 869 & 963 \\
\# of stocks traded per virtual server & 183 & 81 & 31 & 16 & 28 & 23 \\
Speed & 12.16 & 1.107 & 0.767 & 0.002 & 1.251 & 0.001 \\
Inventory & $100.0 \%$ & $100.0 \%$ & $99.2 \%$ & $13.6 \%$ & $75.0 \%$ & $75.0 \%$ \\
\# of quotes per stock-day & 6 & 8 & 17 & 74 & 25 & 126 \\
Volume share per stock-day & $0.07 \%$ & $0.17 \%$ & $0.32 \%$ & $0.73 \%$ & $0.56 \%$ & $0.92 \%$ \\
\hline
\end{tabular}




\section{Table II Classification of traders}

This table shows the traders' classification proposed in this paper. Specifically, we split all traders into 12 groups on a stock-day basis. To split traders, we use information from the continuous trading period on the same day. First, we divide all traders into three groups based on their speed (minimum time elapsed between two consecutive orders for the same stock): FAST, MODERATE, and SLOW. Second, we divide each speed group into four subgroups based on the traders' inventory (the absolute ratio of cumulative buy minus cumulative sell volume to cumulative buy plus sell volume at the end of the day): LARGE, MEDIUM, SMALL, and NOTRADE. The characteristics are given per group on a stock-day basis for the period of April and May 2013 for the 97 stocks from TOPIX100. Order flow data, with order IDs as well as virtual server IDs, are provided by the Tokyo Stock Exchange.

\begin{tabular}{|l|l|l|}
\hline FAST & $\begin{array}{l}\text { Traders with speed below the median } \\
\text { (excluding all trader-stock-days for which the minimum speed is higher than } 60 \text { seconds) }\end{array}$ \\
\hline MODERATE & $\begin{array}{l}\text { Traders with speed above the median } \\
\text { (excluding all trader-stock-days for which the minimum speed is higher than } 60 \text { seconds) } \\
\text { Traders with speed greater than } 60 \text { seconds }\end{array}$ \\
\hline SLOW & LARGE & $\begin{array}{l}\text { Trader's inventory equals } 100 \% \\
\text { Trader's inventory above the median and less than } 100 \% \\
\text { (excluding all trader-stock-days for which the inventory equals 100\%) }\end{array}$ \\
MEDIUM & $\begin{array}{l}\text { Trader's inventory below the median and less than } 100 \% \\
\text { (excluding all trader-stock-days for which the inventory equals 100\%) }\end{array}$ \\
SMALL & Trader submits orders that are not filled (zero trades - only quotes) \\
\hline NOTRADE &
\end{tabular}




\section{Table III Description of traders' characteristics}

This table shows summary statistics for the classification of the traders during the continuous trading period according to the scheme proposed in Table II using information about speed and inventory from the same day's continuous trading period. Panel A shows the medians of the $1 \%, 5 \%$, and $10 \%$ percentiles of the trader-stock-day speed distribution, and the median minimum speed (used for classification of traders). Panel B shows the median number of traders, median end-of-day inventory (used for classification of traders), median average intraday inventory and its volatility, and the median number of times during the day that the inventory crosses zero, per trader-stock-day. These characteristics are presented per group for the period of April and May 2013, for the 97 stocks from TOPIX100. Order flow data, with order IDs as well as virtual server IDs, are provided by the Tokyo Stock Exchange.

\begin{tabular}{|c|c|c|c|c|c|c|}
\hline \multicolumn{7}{|c|}{ Panel A: Speed of traders } \\
\hline Speed & Inventory & \# of traders & Min Speed & $\begin{array}{l}\text { Median of } \\
\text { P1 Speed }\end{array}$ & P5 Speed & P10 Speed \\
\hline FAST & $\begin{array}{l}\text { LARGE } \\
\text { MEDIUM } \\
\text { SMALL (HFTs) } \\
\text { NOTRADE }\end{array}$ & $\begin{array}{l}79 \\
84 \\
84 \\
38\end{array}$ & $\begin{array}{l}0.008 \\
0.005 \\
0.004 \\
0.009\end{array}$ & $\begin{array}{l}0.008 \\
0.007 \\
0.007 \\
0.009\end{array}$ & $\begin{array}{l}0.019 \\
0.028 \\
0.025 \\
0.020\end{array}$ & $\begin{array}{l}0.065 \\
0.252 \\
0.120 \\
0.046\end{array}$ \\
\hline MODERATE & $\begin{array}{l}\text { LARGE } \\
\text { MEDIUM } \\
\text { SMALL } \\
\text { NOTRADE }\end{array}$ & $\begin{array}{l}95 \\
76 \\
71 \\
48\end{array}$ & $\begin{array}{l}5.004 \\
4.003 \\
3.298 \\
6.801\end{array}$ & $\begin{array}{l}5.005 \\
4.010 \\
3.383 \\
6.801\end{array}$ & $\begin{array}{l}5.993 \\
7.893 \\
7.422 \\
7.586\end{array}$ & $\begin{array}{c}8.979 \\
15.815 \\
15.057 \\
9.544\end{array}$ \\
\hline SLOW & $\begin{array}{l}\text { LARGE } \\
\text { MEDIUM } \\
\text { SMALL } \\
\text { NOTRADE }\end{array}$ & $\begin{array}{l}212 \\
39 \\
33 \\
37 \\
\end{array}$ & $\begin{array}{c}1,288.993 \\
540.659 \\
531.157 \\
333.359 \\
\end{array}$ & $\begin{array}{c}1,288.993 \\
540.659 \\
531.157 \\
333.359 \\
\end{array}$ & $\begin{array}{c}1,288.993 \\
540.659 \\
531.157 \\
333.359 \\
\end{array}$ & $\begin{array}{c}1,288.993 \\
543.852 \\
535.923 \\
333.651 \\
\end{array}$ \\
\hline \multicolumn{7}{|c|}{ Panel B: Inventory of traders } \\
\hline Speed & Inventory & \# of traders & $\begin{array}{l}\text { End-of-day } \\
\text { Inventory }\end{array}$ & $\begin{array}{l}\text { Median of } \\
\text { Intraday } \\
\text { inventory }\end{array}$ & $\begin{array}{c}\text { Volatility of } \\
\text { intraday } \\
\text { inventory }\end{array}$ & $\begin{array}{l}\text { \# of zero } \\
\text { crossings }\end{array}$ \\
\hline FAST & $\begin{array}{l}\text { LARGE } \\
\text { MEDIUM } \\
\text { SMALL (HFTs) } \\
\text { NOTRADE }\end{array}$ & $\begin{array}{l}79 \\
84 \\
84 \\
38\end{array}$ & $\begin{array}{l}100.00 \% \\
66.67 \% \\
15.15 \%\end{array}$ & $\begin{array}{l}68.75 \% \\
36.97 \% \\
15.33 \%\end{array}$ & $\begin{array}{c}32.06 \% \\
20.90 \% \\
9.50 \%\end{array}$ & $\begin{array}{l}0 \\
0 \\
2\end{array}$ \\
\hline MODERATE & $\begin{array}{l}\text { LARGE } \\
\text { MEDIUM } \\
\text { SMALL } \\
\text { NOTRADE }\end{array}$ & $\begin{array}{l}95 \\
76 \\
71 \\
48\end{array}$ & $\begin{array}{l}100.00 \% \\
65.52 \% \\
16.67 \%\end{array}$ & $\begin{array}{l}75.00 \% \\
37.29 \% \\
18.19 \%\end{array}$ & $\begin{array}{l}33.33 \% \\
21.16 \% \\
11.08 \%\end{array}$ & $\begin{array}{l}0 \\
1 \\
2\end{array}$ \\
\hline SLOW & $\begin{array}{l}\text { LARGE } \\
\text { MEDIUM } \\
\text { SMALL } \\
\text { NOTRADE } \\
\end{array}$ & $\begin{array}{l}212 \\
39 \\
33 \\
37 \\
\end{array}$ & $\begin{array}{l}100.00 \% \\
65.12 \% \\
15.56 \%\end{array}$ & $\begin{array}{c}100.00 \% \\
42.11 \% \\
25.00 \%\end{array}$ & $\begin{array}{l}35.36 \% \\
21.88 \% \\
17.35 \%\end{array}$ & $\begin{array}{l}0 \\
1 \\
1\end{array}$ \\
\hline
\end{tabular}




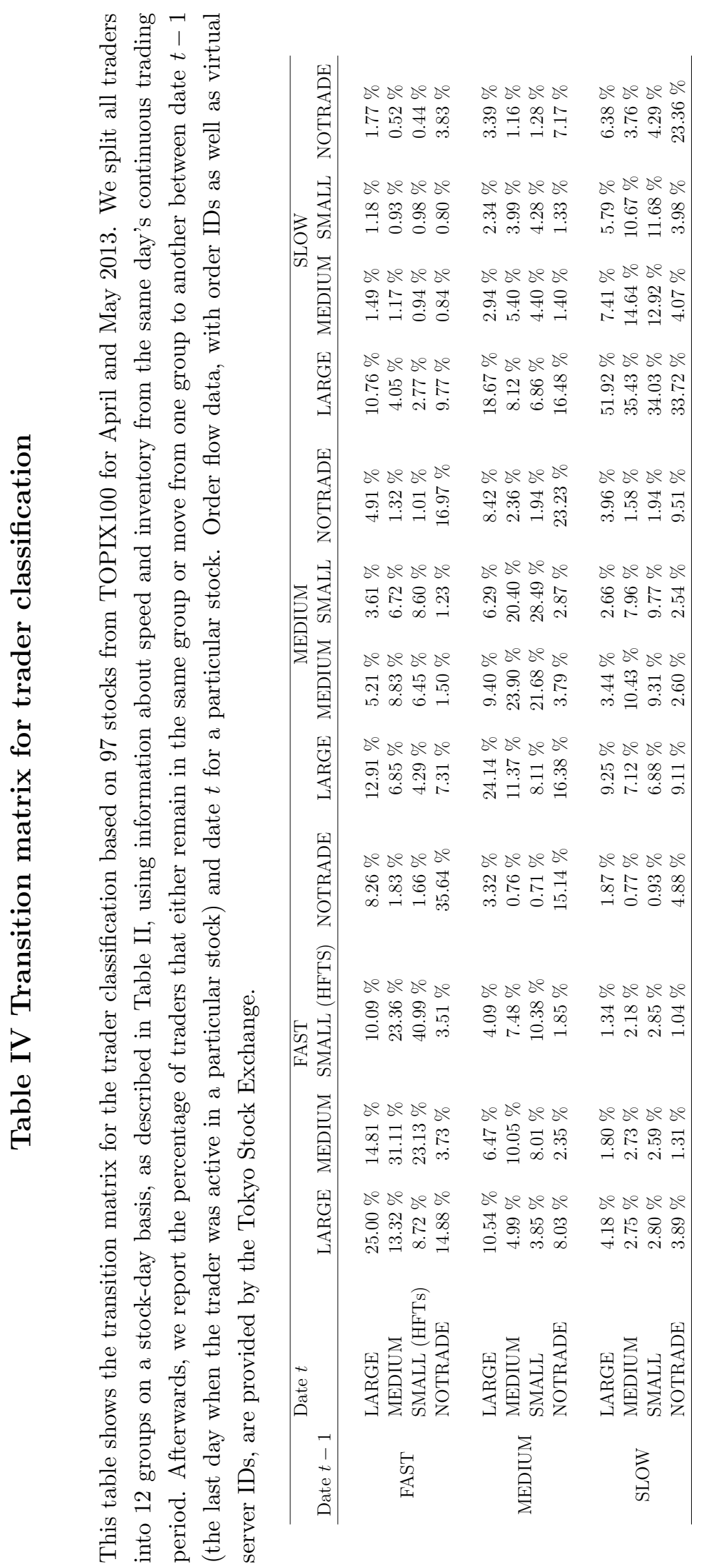




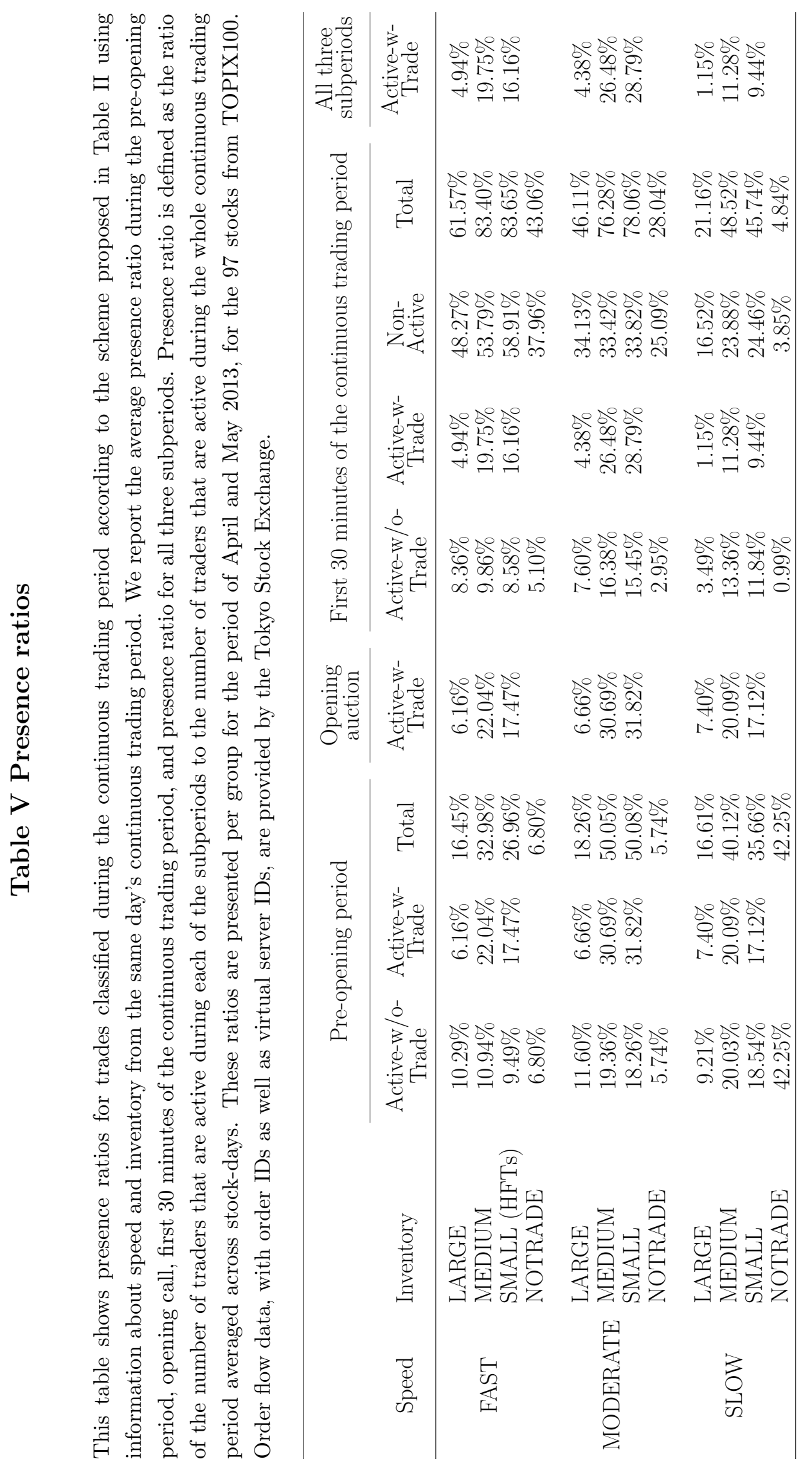




\section{Table VI Quoting activity}

This table shows proportion of quoting activity (new orders, revisions, and cancellations) by 12 trader groups for the pre-opening period and during the first 30 minutes of the continuous trading period. Traders are classified according to the scheme proposed in Table II using information about speed and inventory from the same day's continuous trading period. We also split traders into three categories: traders that do not participate in the pre-opening period (Non-Active), traders that participate during the pre-opening period, but do not trade in the opening call auction (Active-w/o-Trade), and traders that participate during the pre-opening period and trade in the opening call auction (Active-w-Trade). Average quoting activity (Panel A) and regression coefficients (Panels B and C, see equations (13) and (14)) are presented per group for the period of April and May 2013, for the 97 stocks from TOPIX100. ***, **, * correspond to 1\%, 5\%, and $10 \%$ significance levels. Order flow data, with order IDs as well as virtual server IDs, are provided by the Tokyo Stock Exchange.

\begin{tabular}{|c|c|c|c|c|c|c|c|c|}
\hline \multicolumn{9}{|c|}{ Panel A: Average quoting activity } \\
\hline \multirow[b]{2}{*}{ Speed } & \multirow[b]{2}{*}{ Inventory } & \multicolumn{3}{|c|}{ Pre-opening period } & \multicolumn{4}{|c|}{ First 30 minutes of the continuous session } \\
\hline & & Active-w/o-Trade & Active-w-Trade & Total & Active-w/o-Trade & Active-w-Trade & Non-Active & Total \\
\hline FAST & $\begin{array}{l}\text { LARGE } \\
\text { MEDIUM } \\
\text { SMALL (HFTs) } \\
\text { NOTRADE }\end{array}$ & $\begin{array}{l}3.52 \% \\
3.59 \% \\
4.98 \% \\
1.10 \%\end{array}$ & $\begin{array}{l}2.23 \% \\
14.31 \% \\
15.60 \%\end{array}$ & $\begin{array}{c}5.75 \% \\
17.90 \% \\
20.58 \% \\
1.10 \%\end{array}$ & $\begin{array}{l}1.25 \% \\
2.00 \% \\
2.54 \% \\
0.36 \%\end{array}$ & $\begin{array}{c}1.52 \% \\
11.09 \% \\
9.23 \%\end{array}$ & $\begin{array}{c}7.16 \% \\
10.06 \% \\
30.28 \% \\
4.71 \%\end{array}$ & $\begin{array}{l}9.93 \% \\
23.15 \% \\
42.05 \% \\
5.07 \%\end{array}$ \\
\hline MODERATE & $\begin{array}{l}\text { LARGE } \\
\text { MEDIUM } \\
\text { SMALL } \\
\text { NOTRADE }\end{array}$ & $\begin{array}{l}2.83 \% \\
4.03 \% \\
3.64 \% \\
0.84 \%\end{array}$ & $\begin{array}{c}2.34 \% \\
12.23 \% \\
15.04 \%\end{array}$ & $\begin{array}{c}5.17 \% \\
16.26 \% \\
18.68 \% \\
0.84 \%\end{array}$ & $\begin{array}{l}0.58 \% \\
0.98 \% \\
0.92 \% \\
0.15 \%\end{array}$ & $\begin{array}{l}0.46 \% \\
2.45 \% \\
3.15 \%\end{array}$ & $\begin{array}{l}2.46 \% \\
2.06 \% \\
2.45 \% \\
1.39 \%\end{array}$ & $\begin{array}{l}3.50 \% \\
5.49 \% \\
6.52 \% \\
1.54 \%\end{array}$ \\
\hline SLOW & $\begin{array}{l}\text { LARGE } \\
\text { MEDIUM } \\
\text { SMALL } \\
\text { NOTRADE }\end{array}$ & $\begin{array}{l}2.48 \% \\
1.39 \% \\
1.11 \% \\
2.01 \% \\
\end{array}$ & $\begin{array}{l}2.84 \% \\
2.24 \% \\
1.63 \%\end{array}$ & $\begin{array}{l}5.32 \% \\
3.63 \% \\
2.74 \% \\
2.01 \% \\
\end{array}$ & $\begin{array}{l}0.27 \% \\
0.23 \% \\
0.17 \% \\
0.07 \% \\
\end{array}$ & $\begin{array}{l}0.08 \% \\
0.18 \% \\
0.13 \%\end{array}$ & $\begin{array}{l}0.93 \% \\
0.28 \% \\
0.26 \% \\
0.15 \% \\
\end{array}$ & $\begin{array}{l}1.28 \% \\
0.69 \% \\
0.56 \% \\
0.22 \% \\
\end{array}$ \\
\hline \multicolumn{9}{|c|}{ Panel B: Quoting activity regression by stock-date } \\
\hline & & \multicolumn{3}{|c|}{ Pre-opening period } & \multicolumn{4}{|c|}{ First 30 minutes of the continuous session } \\
\hline Speed & Inventory & Active-w/o-Trade & Active-w-Trade & Total & Active-w/o-Trade & Active-w-Trade & Non-Active & Total \\
\hline FAST & $\begin{array}{l}\text { LARGE } \\
\text { MEDIUM } \\
\text { SMALL (HFTs) } \\
\text { NOTRADE }\end{array}$ & $\begin{array}{l}1.89^{* * *} \\
1.97^{* * *} \\
3.36^{* * *} \\
-0.53^{* * *}\end{array}$ & $\begin{array}{l}0.61^{* * * *} \\
12.69^{* * * *} \\
13.98^{* * *} *\end{array}$ & $\begin{array}{l}0.86^{* * *} \\
6.94^{* * *} \\
8.28^{* * *} \\
-0.91^{* * *}\end{array}$ & $\begin{array}{c}1.12^{* * *} \\
1.86^{* * *} \\
2.41^{* * *} \\
0.23^{* *}\end{array}$ & $\begin{array}{c}1.39^{* * *} * \\
10.95^{* * *} \\
9.09^{* * *}\end{array}$ & $\begin{array}{c}7.02^{* * *} \\
9.93^{* * *} \\
30.15^{* * *} \\
4.58^{* * *}\end{array}$ & $\begin{array}{c}3.20^{* * * *} \\
7.61^{* * *} \\
13.91^{* * *} * \\
2.43^{* * *}\end{array}$ \\
\hline MODERATE & $\begin{array}{l}\text { LARGE } \\
\text { MEDIUM } \\
\text { SMALL } \\
\text { NOTRADE }\end{array}$ & $\begin{array}{l}1.21^{* * * *} \\
2.41^{* * *} \\
2.02^{* * *} \\
-0.79^{* * *}\end{array}$ & $\begin{array}{c}0.72^{* * *} \\
10.61^{* * *} \\
13.42^{* * *}\end{array}$ & $\begin{array}{l}0.58^{* * *} \\
6.12^{* * *} \\
7.33^{* * *} \\
-1.17^{* * *}\end{array}$ & $\begin{array}{c}0.44^{* * * *} \\
0.85^{* * *} \\
0.79^{* * *} \\
0.01\end{array}$ & $\begin{array}{l}0.32^{* * *} * \\
2.32^{* * *} \\
3.01^{* * *}\end{array}$ & $\begin{array}{l}2.32^{* * *} \\
1.93^{* * *} \\
2.32^{* * *} \\
1.25^{* * *}\end{array}$ & $\begin{array}{l}1.06^{* * *} \\
1.72^{* * *} \\
2.06^{* * *} \\
0.66^{* * *}\end{array}$ \\
\hline \multirow[t]{5}{*}{ SLOW } & $\begin{array}{l}\text { LARGE } \\
\text { MEDIUM } \\
\text { SMALL } \\
\text { NOTRADE } \\
\end{array}$ & $\begin{array}{c}0.86^{* * *} \\
-0.23^{* * *} \\
-0.52^{* * *} \\
0.38^{* * *} \\
\end{array}$ & $\begin{array}{l}1.21^{* * *} \\
0.61^{* * *}\end{array}$ & $\begin{array}{l}0.65^{* * *} \\
-0.20^{* *} \\
-0.64^{* * *}\end{array}$ & $\begin{array}{c}0.13^{* * * *} \\
0.09^{* * *} \\
0.04^{* * *} \\
-0.07^{* * *} \\
\end{array}$ & $\begin{array}{c}-0.05^{* * *} \\
0.05^{* * *}\end{array}$ & $\begin{array}{c}0.80^{* * *} \\
0.15^{* * *} \\
0.12^{* * *} \\
0.02 \\
\end{array}$ & $\begin{array}{l}0.32^{* * *} * \\
0.12^{* * *} \\
0.08^{* * *}\end{array}$ \\
\hline & $\begin{array}{l}\text { Constant } \\
\# \text { obs } \\
\text { Adj } R^{2} \\
\text { Clustered St.Err. }\end{array}$ & \multicolumn{2}{|c|}{$\begin{array}{c}1.63^{* * *} \\
66,885 \\
0.510 \\
\text { By stock } \\
\end{array}$} & $\begin{array}{c}2.01^{* * * *} \\
66,885 \\
0.281 \\
\text { By stock } \\
\end{array}$ & \multicolumn{3}{|c|}{$\begin{array}{c}0.13^{* * * *} \\
105,105 \\
0.757 \\
\text { By stock }\end{array}$} & $\begin{array}{c}0.11 * * * \\
105,105 \\
0.391 \\
\text { By stock }\end{array}$ \\
\hline & \multicolumn{8}{|c|}{ Panel C: Quoting activity regression for HFT by stock-date } \\
\hline & & \multicolumn{3}{|c|}{ Pre-opening period } & \multicolumn{4}{|c|}{ First 30 minutes of the continuous session } \\
\hline & & $(1)$ & $(2)$ & $(3)$ & $(4)$ & $(5)$ & $(6)$ & $(7)$ \\
\hline \multirow[t]{2}{*}{ FAST / SMALL (HFTs) } & $\begin{array}{l}\text { Non-Active } \\
\text { Active-w/o-Trade } \\
\text { Active-w-Trade } \\
\text { Total } \\
\end{array}$ & 0.23 & $11.39^{* * *}$ & $6.11^{* * *}$ & $28.10^{* * *}$ & $-0.50^{* *}$ & $6.39^{* * *}$ & $12.09^{* * *}$ \\
\hline & $\begin{array}{l}\text { Constant } \\
\text { \# obs } \\
\text { Adj } R^{2} \\
\text { Clustered St.Err. }\end{array}$ & $\begin{array}{c}4.75 * * * \\
66,885 \\
0.000 \\
\text { By stock } \\
\end{array}$ & $\begin{array}{c}4.22^{* * * *} \\
66,885 \\
0.132 \\
\text { By stock } \\
\end{array}$ & $\begin{array}{c}4.18^{* * *} \\
66,885 \\
0.072 \\
\text { By stock } \\
\end{array}$ & $\begin{array}{c}2.18^{* * *} \\
105,105 \\
0.553 \\
\text { By stock } \\
\end{array}$ & $\begin{array}{c}3.05^{* * *} \\
105,105 \\
0.000 \\
\text { By stock } \\
\end{array}$ & $\begin{array}{c}2.84^{* * * *} \\
105,105 \\
0.029 \\
\text { By stock } \\
\end{array}$ & $\begin{array}{c}1.93^{* * *} * \\
105,105 \\
0.288 \\
\text { By stock }\end{array}$ \\
\hline
\end{tabular}




\section{Table VII Trading activity}

This table shows the proportion of trading activity (buy+sell volume) carried out by nine trader groups (the NOTRADE category is omitted as these traders do not trade during the stock-day), for the opening call auction and during the first 30 minutes of the continuous trading session. The grand total across trader groups is $200 \%$. Traders are classified according to the scheme proposed in Table II using information about speed and inventory from the same day's continuous session. We also split traders into three categories: traders that do not participate in the pre-opening period (Non-Active), traders that participate during the pre-opening period, but do not trade at the opening call auction (Active-w/o-Trade), and traders that participate during the pre-opening period and trade at the opening call auction (Active-w-Trade). Average trading activity (Panel A) and regression coefficients (Panels B and C, see equations (13) and (14)) are presented per group for the period of April and May 2013, for the 97 stocks from TOPIX100. Order flow data, with order IDs as well as virtual server IDs, are provided by the Tokyo Stock Exchange.

\begin{tabular}{|c|c|c|c|c|c|c|}
\hline \multicolumn{7}{|c|}{ Panel A: Average trading activity } \\
\hline \multirow[b]{2}{*}{ Speed } & \multirow[b]{2}{*}{ Inventory } & \multirow{2}{*}{$\frac{\text { Opening auction }}{\text { Active-w-Trade }}$} & \multicolumn{4}{|c|}{ First 30 minutes of the continuous session } \\
\hline & & & Active-w/o-Trade & Active-w-Trade & Non-Active & Total \\
\hline FAST & $\begin{array}{l}\text { LARGE } \\
\text { MEDIUM } \\
\text { SMALL (HFTs) }\end{array}$ & $\begin{array}{l}10.37 \% \\
53.64 \% \\
36.65 \%\end{array}$ & $\begin{array}{l}1.44 \% \\
3.65 \% \\
4.69 \%\end{array}$ & $\begin{array}{l}3.47 \% \\
23.11 \% \\
19.79 \%\end{array}$ & $\begin{array}{l}6.75 \% \\
15.80 \% \\
44.40 \%\end{array}$ & $\begin{array}{l}11.66 \% \\
42.56 \% \\
68.88 \%\end{array}$ \\
\hline MODERATE & $\begin{array}{l}\text { LARGE } \\
\text { MEDIUM } \\
\text { SMALL }\end{array}$ & $\begin{array}{c}9.85 \% \\
30.36 \% \\
25.67 \%\end{array}$ & $\begin{array}{l}1.74 \% \\
4.03 \% \\
3.67 \%\end{array}$ & $\begin{array}{c}1.56 \% \\
9.78 \% \\
11.27 \%\end{array}$ & $\begin{array}{c}5.24 \% \\
7.59 \% \\
11.03 \%\end{array}$ & $\begin{array}{c}8.54 \% \\
21.40 \% \\
25.97 \%\end{array}$ \\
\hline SLOW & $\begin{array}{l}\text { LARGE } \\
\text { MEDIUM } \\
\text { SMALL }\end{array}$ & $\begin{array}{l}18.62 \% \\
9.57 \% \\
5.28 \% \\
\end{array}$ & $\begin{array}{l}1.83 \% \\
1.61 \% \\
1.02 \% \\
\end{array}$ & $\begin{array}{l}0.64 \% \\
1.24 \% \\
0.75 \% \\
\end{array}$ & $\begin{array}{l}8.51 \% \\
2.80 \% \\
2.59 \% \\
\end{array}$ & $\begin{array}{l}10.98 \% \\
5.65 \% \\
4.36 \% \\
\end{array}$ \\
\hline \multicolumn{7}{|c|}{ Panel B: Trading activity regression by stock-date } \\
\hline & & Opening auction & \multicolumn{4}{|c|}{ First 30 minutes of the continuous session } \\
\hline Speed & Inventory & $\overline{\text { Active-w-Trade }}$ & Active-w/o-Trade & Active-w-Trade & Non-Active & Total \\
\hline FAST & $\begin{array}{l}\text { LARGE } \\
\text { MEDIUM } \\
\text { SMALL (HFTs) }\end{array}$ & $\begin{array}{c}5.09^{* * *} \\
48.36^{* * *} \\
31.37^{* * *}\end{array}$ & $\begin{array}{l}0.69^{* * *} \\
2.90^{* * *} \\
3.95^{* * *}\end{array}$ & $\begin{array}{l}2.73^{* * * *} \\
22.36^{* * *} \\
19.05^{* * *}\end{array}$ & $\begin{array}{l}6.00^{* * *} * \\
15.05^{* * *} \\
43.65^{* * *}\end{array}$ & $\begin{array}{c}2.44^{* * *} \\
12.73^{* * *} \\
21.51^{* * *}\end{array}$ \\
\hline MODERATE & $\begin{array}{l}\text { LARGE } \\
\text { MEDIUM } \\
\text { SMALL }\end{array}$ & $\begin{array}{l}4.57^{* * *} \\
25.08^{* * *} \\
20.39^{* * *}\end{array}$ & $\begin{array}{l}1.00^{* * * *} \\
3.29^{* * *} * \\
2.93^{* * *}\end{array}$ & $\begin{array}{c}0.81^{* * *} \\
9.03^{* * *} \\
10.52^{* * *}\end{array}$ & $\begin{array}{c}4.49^{* * *} \\
6.84^{* * *} \\
10.28^{* * *}\end{array}$ & $\begin{array}{l}1.40^{* * *} \\
5.68^{* * *} \\
7.20^{* * *}\end{array}$ \\
\hline \multirow[t]{5}{*}{ SLOW } & $\begin{array}{l}\text { LARGE } \\
\text { MEDIUM } \\
\text { SMALL } \\
\end{array}$ & $\begin{array}{c}13.34^{* * *} \\
4.29^{* * *}\end{array}$ & $\begin{array}{l}1.09^{* * * *} \\
0.86^{* * *} \\
0.28^{* * *} \\
\end{array}$ & $\begin{array}{c}-0.11^{* * *} \\
0.49^{* * *}\end{array}$ & $\begin{array}{l}7.76^{* * *} \\
2.05^{* * *} \\
1.84^{* * *} \\
\end{array}$ & $\begin{array}{l}2.21^{* * *} \\
0.43^{* * *}\end{array}$ \\
\hline & $\begin{array}{l}\text { Constant } \\
\# \text { obs } \\
\text { Adj } R^{2} \\
\text { Clustered St.Err. }\end{array}$ & $\begin{array}{c}5.28^{* * *} \\
28,665 \\
0.493 \\
\text { By stock }\end{array}$ & & $\begin{array}{c}0.75^{* * * *} \\
85,995 \\
0.684 \\
\text { By stock }\end{array}$ & & $\begin{array}{c}1.45^{* * * *} \\
85,995 \\
0.356 \\
\text { By stock }\end{array}$ \\
\hline & \multicolumn{6}{|c|}{ Panel C: Trading activity regression for HFT by stock-date } \\
\hline & & Opening auction & \multicolumn{4}{|c|}{ First 30 minutes of the continuous session } \\
\hline & & $(1)$ & $(2)$ & $(3)$ & $(4)$ & $(5)$ \\
\hline FAST / SMALL (HFTs) & $\begin{array}{l}\text { Non-Active } \\
\text { Active-w/o-Trade } \\
\text { Active-w-Trade } \\
\text { Total } \\
\end{array}$ & $16.23^{* * *}$ & $38.42^{* * *}$ & $-2.82^{* * *}$ & $12.86^{* * *}$ & $17.50^{* * *}$ \\
\hline & $\begin{array}{l}\text { Constant } \\
\# \text { obs } \\
\text { Adj } R^{2} \\
\text { Clustered St.Err. }\end{array}$ & $\begin{array}{c}20.42^{* * * *} \\
28,665 \\
0.057 \\
\text { By stock }\end{array}$ & $\begin{array}{c}5.98^{* * *} \\
85,995 \\
0.419 \\
\text { By stock } \\
\end{array}$ & $\begin{array}{c}7.51^{* * * *} \\
85,995 \\
0.002 \\
\text { By stock }\end{array}$ & $\begin{array}{c}6.93^{* * *} \\
85,995 \\
0.047 \\
\text { By stock } \\
\end{array}$ & $\begin{array}{c}5.46 \text { *** } \\
85,995 \\
0.241 \\
\text { By stock }\end{array}$ \\
\hline
\end{tabular}




\section{Table VIII Weighted price discovery}

This table presents the summary statistics for the weighted price discovery contribution $(W P C)$ during the pre-opening period and first 30 minutes of the continuous trading period (see equation (7)). WPC is attributable to aggressive orders. We divide all traders into 12 groups on a stock-day basis, as described in Table II, using information about speed and inventory from the same day's continuous session. We also split traders into three categories: traders that are not active during the pre-opening period (Non-Active), traders that participate during the pre-opening period, but do not trade at the opening call auction (Active-w/oTrade), and traders that participate during the pre-opening period and trade at the opening call auction (Active-w-Trade). Average WPC (Panel A) and regression coefficients (Panels B and C, see equations (13) and (14)) are presented per group for the period of April and May 2013, for the 97 stocks from TOPIX100. ***, **, * correspond to $1 \%, 5 \%$, and $10 \%$ significance levels. Order flow data, with order IDs as well as virtual server IDs, are provided by the Tokyo Stock Exchange.

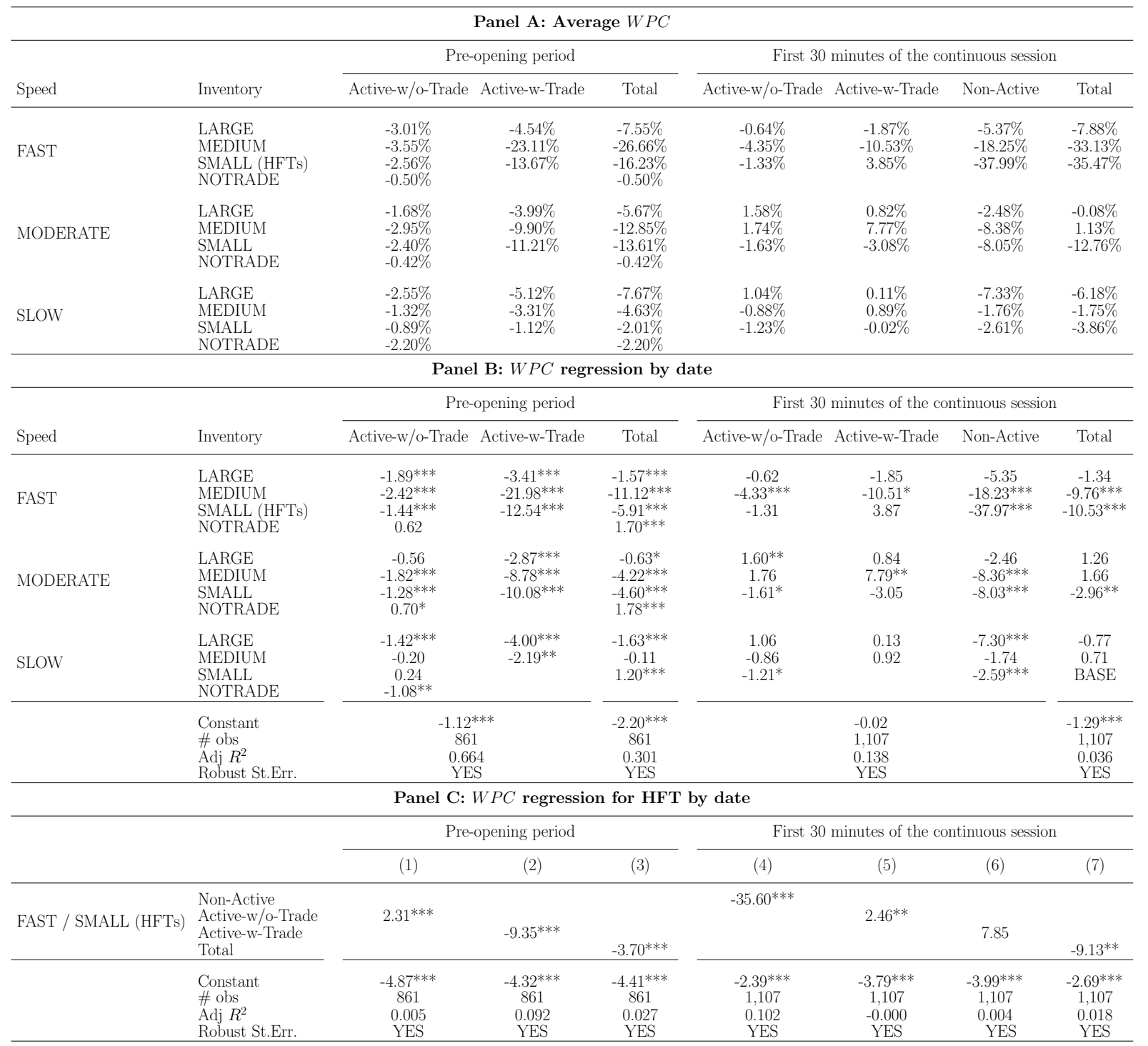




\section{Table IX Net liquidity provision}

This table shows the net liquidity provision among nine trader groups (the NOTRADE category is omitted as these traders do not trade during the stock-day), for the opening call auction and during the first 30 minutes of the continuous trading session. Traders are classified according to the scheme proposed in Table II using information about speed and inventory from the same day's continuous session. We also split traders into three categories: traders that do not participate in the pre-opening period (Non-Active), traders that participate during the pre-opening period, but do not trade at the opening call auction (Active-w/oTrade), and traders that participate during the pre-opening period and trade at the opening call auction (Active-w-Trade). Average net liquidity provision (Panel A) and regression coefficients (Panels B and C, see equations (13) and (14)) are presented per group for the period of April and May 2013, for the 97 stocks from TOPIX100. ${ }^{* * *},{ }^{* *}, *$ correspond to $1 \%, 5 \%$, and $10 \%$ significance levels. Order flow data, with order IDs as well as virtual server IDs, are provided by the Tokyo Stock Exchange.

\begin{tabular}{|c|c|c|c|c|c|c|}
\hline \multicolumn{7}{|c|}{ Panel A: Average net liquidity provision } \\
\hline \multirow[b]{2}{*}{ Speed } & \multirow[b]{2}{*}{ Inventory } & \multirow{2}{*}{$\frac{\text { Opening auction }}{\text { Active-w-Trade }}$} & \multicolumn{4}{|c|}{ First 30 minutes of the continuous session } \\
\hline & & & Active-w/o-Trade & Active-w-Trade & Non-Active & Total \\
\hline FAST & $\begin{array}{l}\text { LARGE } \\
\text { MEDIUM } \\
\text { SMALL (HFTs) }\end{array}$ & $\begin{array}{r}1.53 \% \\
3.13 \% \\
-0.99 \%\end{array}$ & $\begin{array}{l}-0.65 \% \\
-1.23 \% \\
-2.43 \%\end{array}$ & $\begin{array}{l}-1.37 \% \\
-7.71 \% \\
-4.90 \%\end{array}$ & $\begin{array}{c}-1.58 \% \\
0.41 \% \\
14.73 \%\end{array}$ & $\begin{array}{l}-3.60 \% \\
-8.53 \% \\
7.40 \%\end{array}$ \\
\hline MODERATE & $\begin{array}{l}\text { LARGE } \\
\text { MEDIUM } \\
\text { SMALL }\end{array}$ & $\begin{array}{l}0.02 \% \\
-2.55 \% \\
-1.22 \%\end{array}$ & $\begin{array}{l}-0.67 \% \\
-1.34 \% \\
-1.12 \%\end{array}$ & $\begin{array}{l}-0.36 \% \\
-2.22 \% \\
-2.05 \%\end{array}$ & $\begin{array}{l}-0.67 \% \\
2.35 \% \\
5.42 \%\end{array}$ & $\begin{array}{l}-1.70 \% \\
-1.21 \% \\
2.25 \%\end{array}$ \\
\hline SLOW & $\begin{array}{l}\text { LARGE } \\
\text { MEDIUM } \\
\text { SMALL }\end{array}$ & $\begin{array}{r}0.42 \% \\
-0.17 \% \\
-0.18 \% \\
\end{array}$ & $\begin{array}{l}-0.81 \% \\
-0.55 \% \\
-0.36 \% \\
\end{array}$ & $\begin{array}{l}-0.14 \% \\
-0.32 \% \\
-0.13 \% \\
\end{array}$ & $\begin{array}{l}4.84 \% \\
1.65 \% \\
1.18 \% \\
\end{array}$ & $\begin{array}{l}3.89 \% \\
0.78 \% \\
0.69 \% \\
\end{array}$ \\
\hline \multicolumn{7}{|c|}{ Panel B: Net liquidity provision regression by stock-date } \\
\hline & & Opening auction & \multicolumn{4}{|c|}{ First 30 minutes of the continuous session } \\
\hline Speed & Inventory & Active-w-Trade & Active-w/o-Trade & Active-w-Trade & Non-Active & Total \\
\hline FAST & $\begin{array}{l}\text { LARGE } \\
\text { MEDIUM } \\
\text { SMALL (HFTs) }\end{array}$ & $\begin{array}{c}1.70^{* * *} \\
3.31^{* * *} \\
-0.81^{* * *}\end{array}$ & $\begin{array}{l}-0.52^{* * *} \\
-1.10^{* * *} \\
-2.31^{* * *}\end{array}$ & $\begin{array}{l}-1.24^{* * *} \\
-7.58^{* * *} \\
-4.78^{* * *}\end{array}$ & $\begin{array}{c}-1.45^{* * *} \\
0.53^{* * *} \\
14.86^{* * *}\end{array}$ & $\begin{array}{l}-1.43^{* * *} \\
-3.07^{* * *} \\
2.23^{* * *}\end{array}$ \\
\hline MODERATE & $\begin{array}{l}\text { LARGE } \\
\text { MEDIUM } \\
\text { SMALL }\end{array}$ & $\begin{array}{c}0.19 \\
-2.37^{* * *} \\
-1.04^{* * *}\end{array}$ & $\begin{array}{l}-0.54^{* * *} \\
-1.21^{* * *} \\
-0.99^{* * *}\end{array}$ & $\begin{array}{l}-0.23^{* * *} \\
-2.09^{* * *} \\
-1.93^{* * *}\end{array}$ & $\begin{array}{c}-0.54^{* * *} \\
2.48^{* * *} \\
5.55^{* * *}\end{array}$ & $\begin{array}{c}-0.79^{* * *} \\
-0.63^{* * *} \\
0.52^{* * *}\end{array}$ \\
\hline \multirow[t]{5}{*}{ SLOW } & $\begin{array}{l}\text { LARGE } \\
\text { MEDIUM } \\
\text { SMALL } \\
\end{array}$ & $\begin{array}{c}0.60^{* *} \\
0.01\end{array}$ & $\begin{array}{l}-0.68^{* * *} \\
-0.42^{* * *} \\
-0.24^{* * *} \\
\end{array}$ & $\begin{array}{c}-0.02 \\
-0.19^{* * *}\end{array}$ & $\begin{array}{l}4.97^{* * *} \\
1.77^{* * *} \\
1.31^{* * *} \\
\end{array}$ & $\begin{array}{c}1.07^{* * *} \\
0.03\end{array}$ \\
\hline & $\begin{array}{l}\text { Constant } \\
\# \text { obs } \\
\text { Adj } R^{2} \\
\text { Clustered St.Err. }\end{array}$ & $\begin{array}{c}-0.18^{* *} \\
26,685 \\
0.016 \\
\text { By stock } \\
\end{array}$ & & $\begin{array}{c}-0.13^{* * *} \\
85,995 \\
0.503 \\
\text { By stock } \\
\end{array}$ & & $\begin{array}{c}0.23^{* * *} \\
85,995 \\
0.071 \\
\text { By stock }\end{array}$ \\
\hline & \multicolumn{6}{|c|}{ Panel C: Net liquidity provision regression for HFT by stock-date } \\
\hline & & Opening auction & \multicolumn{4}{|c|}{ First 30 minutes of the continuous session } \\
\hline & & $(1)$ & $(2)$ & (3) & $(4)$ & $(5)$ \\
\hline \multirow[t]{2}{*}{ FAST / SMALL (HFTs) } & $\begin{array}{l}\text { Non-Active } \\
\text { Active-w/o-Trade } \\
\text { Active-w-Trade } \\
\text { Total } \\
\end{array}$ & $-1.11^{* * *}$ & $15.30^{* * *}$ & $-2.53^{* * *}$ & $-5.09 * * *$ & $2.77^{* * *}$ \\
\hline & $\begin{array}{l}\text { Constant } \\
\# \text { obs } \\
\text { Adj } R^{2} \\
\text { Clustered St.Err. }\end{array}$ & $\begin{array}{c}0.12^{* * *} \\
26,685 \\
0.001 \\
\text { By stock }\end{array}$ & $\begin{array}{c}-0.57^{* * * *} \\
85,995 \\
0.291 \\
\text { By stock } \\
\end{array}$ & $\begin{array}{c}0.09^{* * *} \\
85,995 \\
0.008 \\
\text { By stock }\end{array}$ & $\begin{array}{c}0.19^{* * *} \\
85,995 \\
0.032 \\
\text { By stock }\end{array}$ & $\begin{array}{c}-0.31^{* * *} \\
85,995 \\
0.026 \\
\text { By stock }\end{array}$ \\
\hline
\end{tabular}




\section{Table X Quoting activity regression in different regimes}

This table shows the change in quoting activity of 12 trader groups during high systematic and idiosyncratic volatility regimes for the pre-opening period and during the first 30 minutes of the continuous trading period. Traders are classified according to the scheme proposed in Table II using information about speed and inventory from the same day's continuous trading period. We also split traders into three categories: traders that do not participate in the pre-opening period (Non-Active), traders that participate during the pre-opening period, but do not trade in the opening call auction (Active-w/o-Trade), and traders that participate during the pre-opening period and trade in the opening call auction (Active-w-Trade). Panel A presents interaction coefficients with systematic volatility dummy, while Panel B presents interaction coefficients with idiosyncratic volatility dummy (see equation (15)) per group for the period of April and May 2013, for the 97 stocks from TOPIX100. ${ }^{* * *},{ }^{* *},{ }^{*}$ correspond to $1 \%, 5 \%$, and $10 \%$ significance levels. Order flow data, with order IDs as well as virtual server IDs, are provided by the Tokyo Stock Exchange.

\begin{tabular}{|c|c|c|c|c|c|c|}
\hline \multirow[b]{2}{*}{ Speed } & \multirow[b]{2}{*}{ Inventory } & \multicolumn{2}{|c|}{ Pre-opening period } & \multicolumn{3}{|c|}{ First 30 minutes of the continuous session } \\
\hline & & Active-w/o-Trade & Active-w-Trade & Active-w/o-Trade & Active-w-Trade & Non-Active \\
\hline \multicolumn{7}{|c|}{ Panel A: Quoting activity regression by date: interaction effects with systematic volatility } \\
\hline FAST & $\begin{array}{l}\text { LARGE } \\
\text { MEDIUM } \\
\text { SMALL (HFTs) } \\
\text { NOTRADE }\end{array}$ & $\begin{array}{c}-0.36^{* * *} \\
0.22 \\
0.96^{* * *} \\
-0.03\end{array}$ & $\begin{array}{l}0.53^{* * *} \\
2.05^{* * *} \\
1.87^{* * *}\end{array}$ & $\begin{array}{c}-0.13 \\
0.14 \\
0.33^{* * *} \\
-0.01\end{array}$ & $\begin{array}{c}-0.09 \\
0.61^{* *} \\
1.11^{* * *}\end{array}$ & $\begin{array}{c}0.14 \\
0.24 \\
-0.32 \\
-0.54^{* * *}\end{array}$ \\
\hline MODERATE & $\begin{array}{l}\text { LARGE } \\
\text { MEDIUM } \\
\text { SMALL } \\
\text { NOTRADE }\end{array}$ & $\begin{array}{c}0.20^{*} \\
0.53^{* * *} \\
0.45^{* * *} \\
0.33^{* * *}\end{array}$ & $\begin{array}{c}0.55^{* * *} \\
0.21 \\
0.32\end{array}$ & $\begin{array}{c}-0.03 \\
0.04 \\
-0.01 \\
0.01\end{array}$ & $\begin{array}{c}0.03 \\
-0.05 \\
-0.10\end{array}$ & $\begin{array}{c}-0.08 \\
0.08^{*} \\
0.26^{* * *} \\
-0.12^{* *}\end{array}$ \\
\hline SLOW & $\begin{array}{l}\text { LARGE } \\
\text { MEDIUM } \\
\text { SMALL } \\
\text { NOTRADE }\end{array}$ & $\begin{array}{c}-0.09 \\
0.18^{* *} \\
0.19^{* *} \\
-0.02\end{array}$ & $\begin{array}{c}0.70^{* * *} \\
-0.02\end{array}$ & $\begin{array}{c}-0.02^{*} \\
0.00 \\
-0.01 \\
0.02^{* *}\end{array}$ & $\begin{array}{c}0.03^{* * *} \\
-0.01\end{array}$ & $\begin{array}{c}-0.04 \\
0.02^{*} \\
0.02 \\
0.01\end{array}$ \\
\hline \multicolumn{7}{|c|}{ Panel B: Quoting activity regression by stock: interaction effects with idiosyncratic volatility } \\
\hline FAST & $\begin{array}{l}\text { LARGE } \\
\text { MEDIUM } \\
\text { SMALL (HFTs) } \\
\text { NOTRADE }\end{array}$ & $\begin{array}{c}0.10 \\
0.71^{* * *} \\
1.60^{* * *} \\
0.17^{*}\end{array}$ & $\begin{array}{l}0.41^{* * *} \\
3.06^{* * *} \\
3.85^{* * *}\end{array}$ & $\begin{array}{c}0.02 \\
0.30^{*} \\
0.62^{* * *} \\
0.06\end{array}$ & $\begin{array}{c}-0.16 \\
0.65 \\
1.88^{* * *}\end{array}$ & $\begin{array}{c}-0.33 \\
-0.63^{* * *} \\
0.81 \\
-0.50^{*}\end{array}$ \\
\hline MODERATE & $\begin{array}{l}\text { LARGE } \\
\text { MEDIUM } \\
\text { SMALL } \\
\text { NOTRADE }\end{array}$ & $\begin{array}{c}0.26^{*} \\
0.33^{* *} \\
0.40^{* * *} \\
0.38^{* * *}\end{array}$ & $\begin{array}{c}0.43^{* * *} \\
-0.04 \\
-0.17\end{array}$ & $\begin{array}{c}-0.01 \\
0.00 \\
0.00 \\
0.03^{* *}\end{array}$ & $\begin{array}{c}0.00 \\
-0.05 \\
-0.05\end{array}$ & $\begin{array}{c}-0.44^{* * *} \\
0.01 \\
0.32^{* * *} \\
-0.35^{* * *}\end{array}$ \\
\hline SLOW & $\begin{array}{l}\text { LARGE } \\
\text { MEDIUM } \\
\text { SMALL } \\
\text { NOTRADE }\end{array}$ & $\begin{array}{c}-0.36^{* * *} \\
0.10 \\
0.03 \\
-0.29^{* *}\end{array}$ & $\begin{array}{c}0.17 \\
-0.20^{*}\end{array}$ & $\begin{array}{c}-0.06^{* * *} \\
-0.02 \\
-0.01 \\
0.02^{* *}\end{array}$ & $\begin{array}{c}0.03^{* * *} \\
-0.01\end{array}$ & $\begin{array}{c}-0.17^{* * *} \\
0.01 \\
-0.00 \\
0.01\end{array}$ \\
\hline
\end{tabular}




\section{Table XI Trading activity regression in different regimes}

This table shows the change in trading activity of nine trader groups during high systematic and idiosyncratic volatility regimes for the opening call and the first 30 minutes of the continuous trading period. Traders are classified according to the scheme proposed in Table II using information about speed and inventory from the same day's continuous trading period. We also split traders into three categories: traders that do not participate in the pre-opening period (Non-Active), traders that participate during the pre-opening period, but do not trade in the opening call auction (Active-w/o-Trade), and traders that participate during the pre-opening period and trade in the opening call auction (Active-w-Trade). Panel A presents interaction coefficients with systematic volatility dummy, while Panel B presents interaction coefficients with idiosyncratic volatility dummy (see equation (15)) per group for the period of April and May 2013, for the 97 stocks from TOPIX100. ${ }^{* * *},{ }^{* *},{ }^{*}$ correspond to $1 \%, 5 \%$, and $10 \%$ significance levels. Order flow data, with order IDs as well as virtual server IDs, are provided by the Tokyo Stock Exchange.

\begin{tabular}{|c|c|c|c|c|c|}
\hline \multirow[b]{2}{*}{ Speed } & \multirow[b]{2}{*}{ Inventory } & \multirow{2}{*}{$\frac{\text { Opening auction }}{\text { Active-w-Trade }}$} & \multicolumn{3}{|c|}{ First 30 minutes of the continuous session } \\
\hline & & & Active-w/o-Trade & Active-w-Trade & Non-Active \\
\hline \multicolumn{6}{|c|}{ Panel A: Trading activity regression by stock-date: interaction effects with systematic volatility } \\
\hline FAST & $\begin{array}{l}\text { LARGE } \\
\text { MEDIUM } \\
\text { SMALL (HFTs) }\end{array}$ & $\begin{array}{c}0.21 \\
2.09^{* *} \\
3.87^{* * *}\end{array}$ & $\begin{array}{c}-0.18 \\
0.05 \\
0.34\end{array}$ & $\begin{array}{c}-0.20 \\
1.51^{* * *} \\
2.24^{* * *}\end{array}$ & $\begin{array}{c}-0.22 \\
0.24 \\
-0.46\end{array}$ \\
\hline MODERATE & $\begin{array}{l}\text { LARGE } \\
\text { MEDIUM } \\
\text { SMALL }\end{array}$ & $\begin{array}{c}-2.94^{* * *} \\
1.43^{*} \\
1.70^{* * *}\end{array}$ & $\begin{array}{l}0.06 \\
0.13 \\
0.09\end{array}$ & $\begin{array}{l}0.08 \\
0.21 \\
0.04\end{array}$ & $\begin{array}{c}0.20 \\
0.48^{* *} \\
0.06\end{array}$ \\
\hline SLOW & $\begin{array}{l}\text { LARGE } \\
\text { MEDIUM } \\
\text { SMALL }\end{array}$ & $\begin{array}{c}1.78^{* * * *} \\
-0.33\end{array}$ & $\begin{array}{l}-0.19^{*} \\
-0.12 \\
-0.03\end{array}$ & $\begin{array}{c}0.09 \\
-0.09\end{array}$ & $\begin{array}{c}-0.49^{* *} \\
0.09 \\
-0.29^{* *}\end{array}$ \\
\hline \multicolumn{6}{|c|}{ Panel B: Trading activity regression by stock-date: interaction effects with idiosyncratic volatility } \\
\hline FAST & $\begin{array}{l}\text { LARGE } \\
\text { MEDIUM } \\
\text { SMALL (HFTs) }\end{array}$ & $\begin{array}{c}-0.53 \\
5.63^{* * *} \\
8.02^{* * *}\end{array}$ & $\begin{array}{c}-0.10 \\
0.66^{* * *} \\
1.00^{* * *}\end{array}$ & $\begin{array}{c}-0.32 \\
3.07 * * * \\
4.88^{* * *}\end{array}$ & $\begin{array}{c}-0.58^{* *} \\
-0.74^{*} \\
-0.58\end{array}$ \\
\hline MODERATE & $\begin{array}{l}\text { LARGE } \\
\text { MEDIUM } \\
\text { SMALL }\end{array}$ & $\begin{array}{l}-0.48 \\
-0.59 \\
1.29^{*}\end{array}$ & $\begin{array}{c}0.00 \\
0.27 \\
0.34^{* *}\end{array}$ & $\begin{array}{l}0.05 \\
0.01 \\
0.07\end{array}$ & $\begin{array}{c}-0.56^{* * * *} \\
0.00 \\
1.48^{* * *}\end{array}$ \\
\hline SLOW & $\begin{array}{l}\text { LARGE } \\
\text { MEDIUM } \\
\text { SMALL } \\
\end{array}$ & $\begin{array}{l}-3.37^{* * *} \\
-1.46^{* * *}\end{array}$ & $\begin{array}{c}-0.27^{* *} \\
-0.23^{* *} \\
-0.10 \\
\end{array}$ & $\begin{array}{c}0.03 \\
-0.07\end{array}$ & $\begin{array}{l}-1.94^{* * *} \\
-0.26^{* *} \\
-0.63^{* * *} \\
\end{array}$ \\
\hline
\end{tabular}




\section{Table XII $W P C$ regression in different regimes}

This table shows the change in $W P C$ of 12 trader groups during high systematic and idiosyncratic volatility regimes for the pre-opening period and during the first 30 minutes of the continuous trading period. Traders are classified according to the scheme proposed in Table II using information about speed and inventory from the same day's continuous trading period. We also split traders into three categories: traders that do not participate in the pre-opening period (Non-Active), traders that participate during the pre-opening period, but do not trade in the opening call auction (Active-w/o-Trade), and traders that participate during the pre-opening period and trade in the opening call auction (Active-w-Trade). Panel A presents interaction coefficients with systematic volatility dummy, while Panel B presents interaction coefficients with idiosyncratic volatility dummy (see equation (15)) per group for the period of April and May 2013, for the 97 stocks from TOPIX100. ${ }^{* * *},{ }^{* *},{ }^{*}$ correspond to $1 \%, 5 \%$, and $10 \%$ significance levels. Order flow data, with order IDs as well as virtual server IDs, are provided by the Tokyo Stock Exchange.

\begin{tabular}{|c|c|c|c|c|c|c|}
\hline \multirow[b]{2}{*}{ Speed } & \multirow[b]{2}{*}{ Inventory } & \multicolumn{2}{|c|}{ Pre-opening period } & \multicolumn{3}{|c|}{ First 30 minutes of the continuous session } \\
\hline & & Active-w/o-Trade & Active-w-Trade & Active-w/o-Trade & Active-w-Trade & Non-Active \\
\hline \multicolumn{7}{|c|}{ Panel A: $W P C$ regression by date: interaction effects with systematic volatility } \\
\hline FAST & $\begin{array}{l}\text { LARGE } \\
\text { MEDIUM } \\
\text { SMALL (HFTs) } \\
\text { NOTRADE }\end{array}$ & $\begin{array}{l}0.01 \\
0.01 \\
0.02 \\
0.02\end{array}$ & $\begin{array}{l}0.00 \\
0.02 \\
0.00\end{array}$ & $\begin{array}{c}-0.02 \\
-0.05 \\
0.02\end{array}$ & $\begin{array}{c}0.03 \\
0.24^{*} \\
0.13\end{array}$ & $\begin{array}{l}-0.05 \\
-0.10 \\
-0.29^{*}\end{array}$ \\
\hline MODERATE & $\begin{array}{l}\text { LARGE } \\
\text { MEDIUM } \\
\text { SMALL } \\
\text { NOTRADE }\end{array}$ & $\begin{array}{c}0.01 \\
-0.01 \\
0.01 \\
0.01\end{array}$ & $\begin{array}{c}-0.01 \\
0.01 \\
-0.02\end{array}$ & $\begin{array}{c}-0.00 \\
0.01 \\
0.00\end{array}$ & $\begin{array}{l}0.01 \\
0.10^{*} \\
-0.02\end{array}$ & $\begin{array}{l}-0.07^{*} \\
-0.06 \\
-0.05\end{array}$ \\
\hline SLOW & $\begin{array}{l}\text { LARGE } \\
\text { MEDIUM } \\
\text { SMALL } \\
\text { NOTRADE }\end{array}$ & $\begin{array}{l}0.02 \\
0.02 \\
0.01 \\
0.01\end{array}$ & $\begin{array}{l}-0.04 \\
-0.00\end{array}$ & $\begin{array}{c}-0.01 \\
0.01 \\
0.01\end{array}$ & $\begin{array}{l}-0.01 \\
-0.00\end{array}$ & $\begin{array}{l}-0.03 \\
-0.00 \\
-0.01\end{array}$ \\
\hline \multicolumn{7}{|c|}{ Panel B: $W P C$ regression by date: interaction effects with idiosincratic volatility } \\
\hline FAST & $\begin{array}{l}\text { LARGE } \\
\text { MEDIUM } \\
\text { SMALL (HFTs) } \\
\text { NOTRADE }\end{array}$ & $\begin{array}{c}-0.01 \\
-0.00 \\
0.00 \\
0.00\end{array}$ & $\begin{array}{c}-0.01 \\
-0.10^{* *} \\
-0.11^{* * *}\end{array}$ & $\begin{array}{c}0.02 \\
-0.04 \\
0.04\end{array}$ & $\begin{array}{c}0.04 \\
-0.06 \\
0.19\end{array}$ & $\begin{array}{c}-0.03 \\
-0.24^{* *} \\
-0.12\end{array}$ \\
\hline MODERATE & $\begin{array}{l}\text { LARGE } \\
\text { MEDIUM } \\
\text { SMALL } \\
\text { NOTRADE }\end{array}$ & $\begin{array}{l}-0.01 \\
-0.02 \\
-0.00 \\
-0.00\end{array}$ & $\begin{array}{c}-0.03 \\
0.04 \\
-0.03\end{array}$ & $\begin{array}{l}0.02 \\
0.02 \\
0.02\end{array}$ & $\begin{array}{c}0.03 \\
0.14^{*} \\
-0.19^{*}\end{array}$ & $\begin{array}{c}-0.05 \\
-0.02 \\
0.00\end{array}$ \\
\hline SLOW & $\begin{array}{l}\text { LARGE } \\
\text { MEDIUM } \\
\text { SMALL } \\
\text { NOTRADE }\end{array}$ & $\begin{array}{l}-0.01 \\
-0.00 \\
-0.01 \\
-0.00 \\
\end{array}$ & $\begin{array}{l}-0.01 \\
-0.03\end{array}$ & $\begin{array}{c}0.05^{* * *} \\
0.04^{*} \\
0.04^{*}\end{array}$ & $\begin{array}{c}0.05^{* * *} \\
0.02\end{array}$ & $\begin{array}{c}0.02 \\
0.05^{*} \\
0.04^{*}\end{array}$ \\
\hline
\end{tabular}




\section{Table XIII Net liquidity provision regression in different regimes}

This table shows the change in net liquidity provision of nine trader groups during high systematic and idiosyncratic volatility regimes for the opening call and the first 30 minutes of the continuous trading period. Traders are classified according to the scheme proposed in Table II using information about speed and inventory from the same day's continuous trading period. We also split traders into three categories: traders that do not participate in the pre-opening period (Non-Active), traders that participate during the preopening period, but do not trade in the opening call auction (Active-w/o-Trade), and traders that participate during the pre-opening period and trade in the opening call auction (Active-w-Trade). Panel A presents interaction coefficients with systematic volatility dummy, while Panel B presents interaction coefficients with idiosyncratic volatility dummy (see equation (15)) per group for the period of April and May 2013, for the 97 stocks from TOPIX100. ***, **, * correspond to $1 \%, 5 \%$, and $10 \%$ significance levels. Order flow data, with order IDs as well as virtual server IDs, are provided by the Tokyo Stock Exchange.

\begin{tabular}{|c|c|c|c|c|c|}
\hline \multirow[b]{2}{*}{ Speed } & \multirow[b]{2}{*}{ Inventory } & \multirow{2}{*}{$\frac{\text { Opening auction }}{\text { Active-w-Trade }}$} & \multicolumn{3}{|c|}{ First 30 minutes of the continuous session } \\
\hline & & & Active-w/o-Trade & Active-w-Trade & Non-Active \\
\hline \multicolumn{6}{|c|}{ Panel A: Net liquidity provision regression by stock-date: interaction effects with systematic volatility } \\
\hline FAST & $\begin{array}{l}\text { LARGE } \\
\text { MEDIUM } \\
\text { SMALL (HFTs) }\end{array}$ & $\begin{array}{l}-0.58 \\
0.45 \\
0.28\end{array}$ & $\begin{array}{c}0.24^{* * *} \\
0.01 \\
-0.02\end{array}$ & $\begin{array}{l}0.23 \\
0.47 \\
-0.07\end{array}$ & $\begin{array}{c}0.21 \\
0.08 \\
-1.18^{* * *}\end{array}$ \\
\hline MODERATE & $\begin{array}{l}\text { LARGE } \\
\text { MEDIUM } \\
\text { SMALL }\end{array}$ & $\begin{array}{r}0.33 \\
0.86 \\
-0.13\end{array}$ & $\begin{array}{l}0.08 \\
0.01 \\
0.03\end{array}$ & $\begin{array}{r}0.04 \\
-0.15 \\
0.18\end{array}$ & $\begin{array}{c}0.30^{* *} \\
0.19 \\
-0.26\end{array}$ \\
\hline SLOW & $\begin{array}{l}\text { LARGE } \\
\text { MEDIUM } \\
\text { SMALL }\end{array}$ & $\begin{array}{c}0.58 \\
-0.31\end{array}$ & $\begin{array}{l}0.08 \\
0.06 \\
0.04\end{array}$ & $\begin{array}{l}0.18 \\
0.06 \\
0.08\end{array}$ & $\begin{array}{c}-0.68^{* * *} \\
-0.12 \\
-0.31^{* *}\end{array}$ \\
\hline \multicolumn{6}{|c|}{ Panel B: Net liquidity provision regression by stock-date: interaction effects with idiosyncratic volatility } \\
\hline FAST & $\begin{array}{l}\text { LARGE } \\
\text { MEDIUM } \\
\text { SMALL (HFTs) }\end{array}$ & $\begin{array}{c}-1.47^{* * *} \\
-1.88 \\
-0.46\end{array}$ & $\begin{array}{c}0.22^{* *} \\
-0.28^{* *} \\
-0.54^{* * *}\end{array}$ & $\begin{array}{l}0.39^{* *} \\
0.69^{*} \\
-0.11\end{array}$ & $\begin{array}{c}0.11 \\
-0.45^{*} \\
-2.21^{* * *}\end{array}$ \\
\hline MODERATE & $\begin{array}{l}\text { LARGE } \\
\text { MEDIUM } \\
\text { SMALL }\end{array}$ & $\begin{array}{c}-1.08 \\
0.79 \\
-0.37\end{array}$ & $\begin{array}{l}-0.01 \\
-0.08 \\
-0.01\end{array}$ & $\begin{array}{c}0.02 \\
-0.10\end{array}$ & $\begin{array}{c}0.23^{*} \\
0.05 \\
1.05^{* * *}\end{array}$ \\
\hline SLOW & $\begin{array}{l}\text { LARGE } \\
\text { MEDIUM } \\
\text { SMALL }\end{array}$ & $\begin{array}{c}0.04 \\
-0.18\end{array}$ & $\begin{array}{l}0.09 \\
0.12 \\
0.10 \\
\end{array}$ & $\begin{array}{c}0.51 \\
-0.06 \\
0.09\end{array}$ & $\begin{array}{c}-1.04^{* * *} \\
-0.24^{*} \\
-0.22 \\
\end{array}$ \\
\hline
\end{tabular}




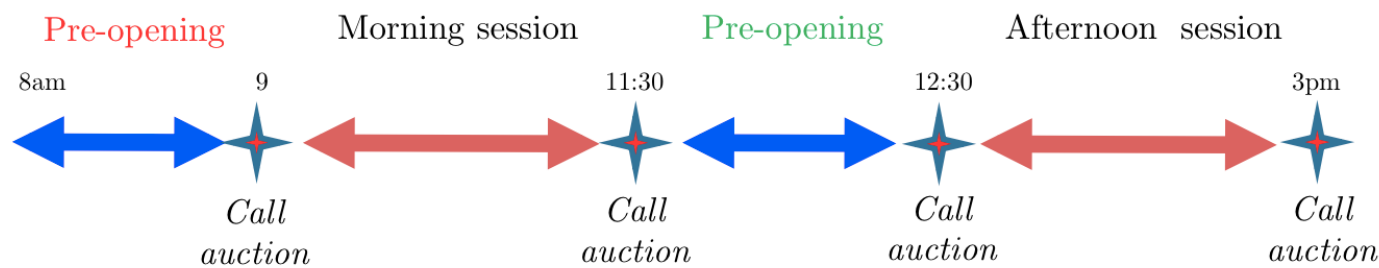

Figure 1. Trading schedule of the TSE. This figure shows the trading schedule of the Tokyo Stock Exchange on a typical trading day. The two separate sessions (morning and afternoon) start, respectively, at 9:00 a.m. and 12.30 p.m. A call auction precedes the subsequent continuous period.

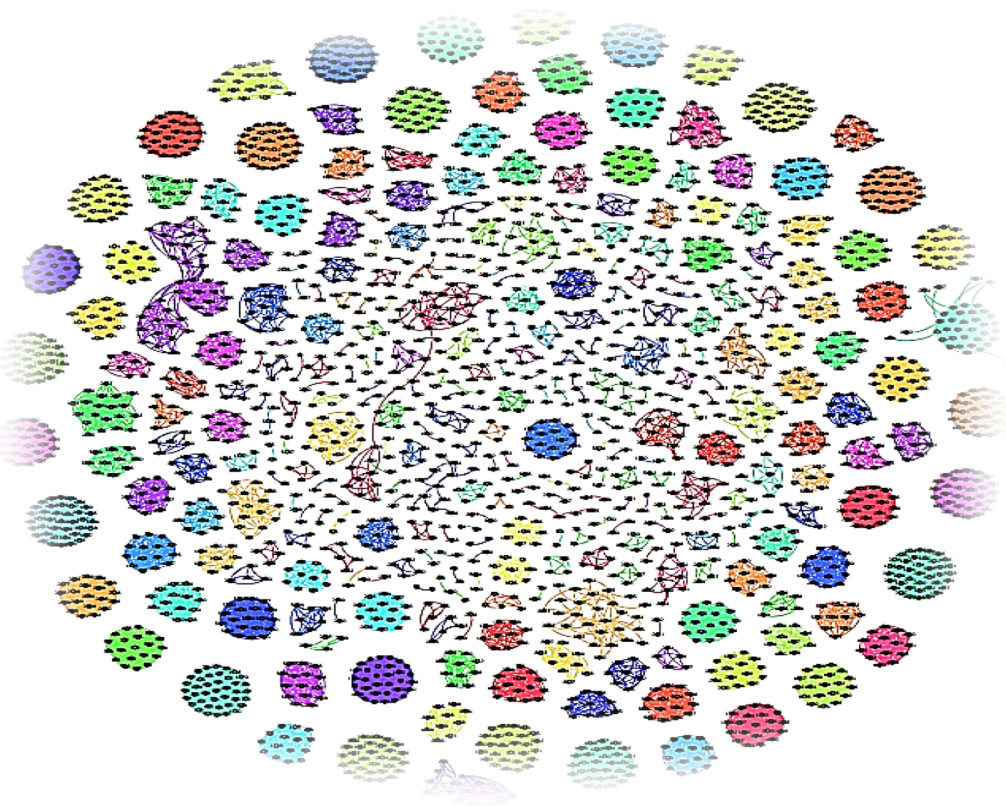

Figure 2. Graphical representation of usage of virtual servers by traders. This graph displays the relation between the number of virtual servers and the number of trading desks, during the period of April and May 2013, on the Tokyo Stock Exchange, for 1,702 stocks. The total number of virtual servers is 5,580 (all the dots in the figure), while the number of trading desks using one or more virtual servers is 3,021 (the colored groups in the figure). Order flow data, with order IDs as well as virtual server IDs, are provided by the Tokyo Stock Exchange. 


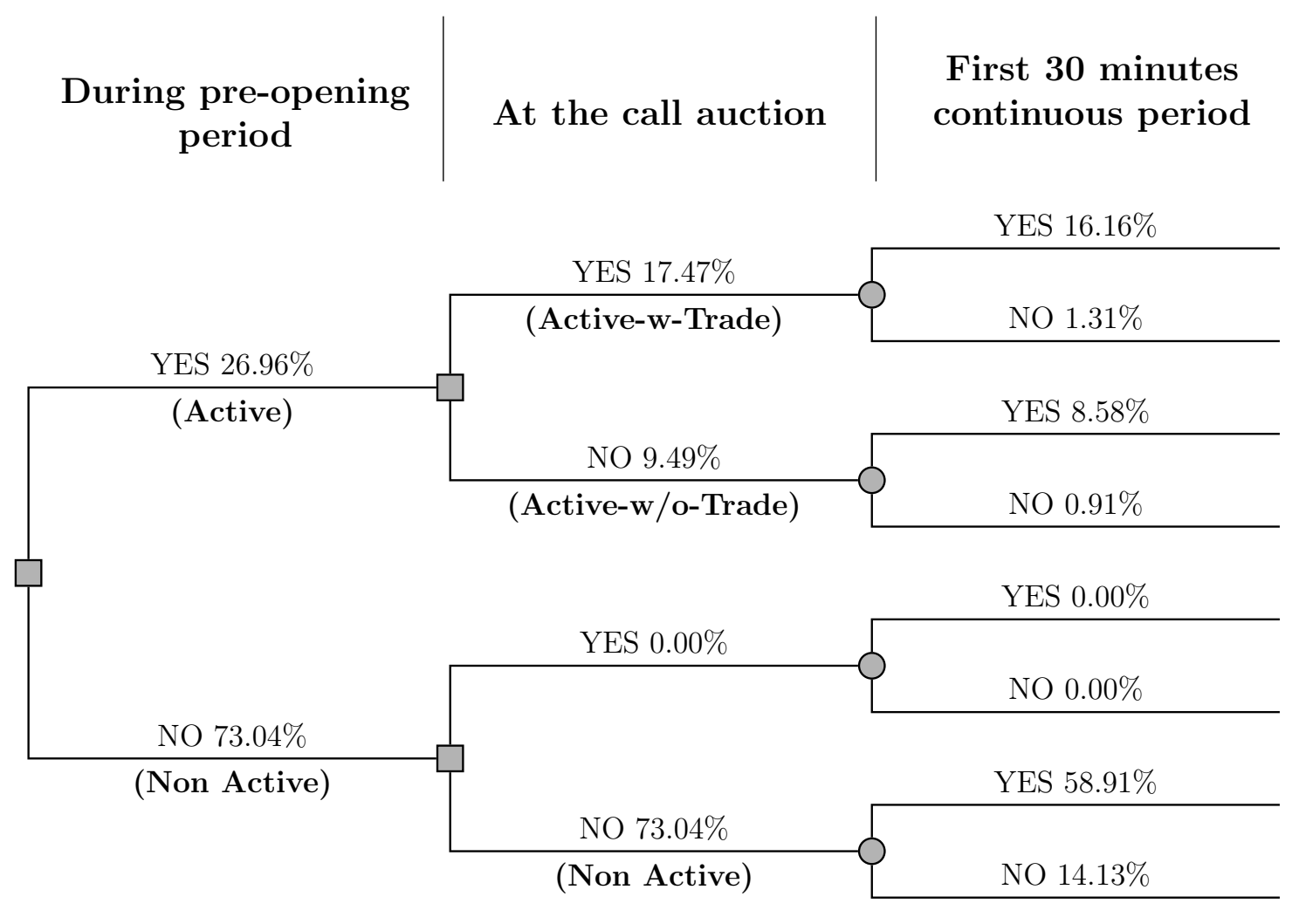

Figure 3. Activity of FAST/SMALL traders (HFTs) during the trading day. This diagram shows activity of FAST/SMALL traders (HFTs) classified during the continuous session according to the scheme proposed in Table II using information about speed and inventory from the same day's continuous session. We report the mean proportions of FAST/SMALL traders (HFTs) based on their decision to participate or not during the pre-opening period, opening call, and first 30 minutes of the continuous trading session. These proportions are presented for the period of April and May 2013, for the 97 stocks from TOPIX100. Order flow data, with order IDs as well as virtual server IDs, are provided by the Tokyo Stock Exchange. 


\title{
Internet Appendix for
}

\author{
"Low-Latency Trading and Price Discovery: Evidence from the \\ Tokyo Stock Exchange in the Pre-Opening and Opening Periods" \\ MARIO BELLIA, LORIANA PELIZZON, MARTI G. SUBRAHMANYAM, \\ JUN UNO and DARYA YUFEROVA
}

\begin{abstract}
This supplemental appendix describes the determination of the best quotes during the preopening period, the configuration of multiple virtual servers (VS) used by a trader and presents additional analyses and robustness checks.
\end{abstract}




\section{Contents}

$\begin{array}{ll}\text { A Best quotes during the pre-opening period } & 3\end{array}$

B Configuration of multiple virtual servers (VSs) used by one trader 5

$\begin{array}{lr}\text { C Speed model estimation } & 6\end{array}$

D An alternative classification scheme: Kirilenko, Kyle, Samadi, and Tuzun (2016) 8

$\begin{array}{ll}\text { E Order flow in the pre-opening period } & 11\end{array}$

$\begin{array}{llr}\text { F Aggressive orders } & 15\end{array}$

$\begin{array}{ll}\text { G Additional analysis for price discovery } & 17\end{array}$

G.1 Regression on WPC per stock-day . . . . . . . . . . . . . . 17

G.2 Price discovery with different reference price . . . . . . . . . . . . 17

G.3 Price discovery by order type . . . . . . . . . . . . . . . . . 19

G.4 Speed of price discovery . . . . . . . . . . . . . . . . . . . . 21

H Unbiasedness of the quoted price $\quad 24$

List of Tables

IA.1 Speed model estimation $\ldots \ldots \ldots \ldots \ldots$

IA.2 Classification scheme proposed by Kirilenko et al. (2016) . . . . . . . . . . . . 9

IA.3 Comparison of classifications . . . . . . . . . . . . . . . 10

IA.4 Aggressive orders during pre-opening period . . . . . . . . . . . . . 16

IA.5 Weighted price discovery regression by stock-date . . . . . . . . . . . . 17

IA.6 Contribution to weighted price discovery: different reference prices . . . . . . . . 18

IA.7 Contribution to weighted price discovery by order type during the pre-opening period 20

IA.8 Aggressive orders across stocks . . . . . . . . . . . . . . . 22

List of Figures

IA.1 Determination of best bid and ask prices during the pre-opening period $\ldots \ldots . .4$

IA.2 Illustration of a possible VS configuration . . . . . . . . . . . . . 5

IA.3 Flow of total order submission in the pre-opening period . . . . . . . . . . . 12

IA.4 Flow of new orders and cancellations in the last 10 minutes of the pre-opening period 13

IA.5 Order flow during the last second of the pre-opening period . . . . . . . . . . . 14

IA.6 Deviation from the opening price . . . . . . . . . . . . . . 21

IA.7 Comparison of the deviation from the opening price between stocks for which highspeed traders have different levels of participation . . . . . . . . . . . 23

IA.8 Tests of unbiasedness regressions of the pre-opening mid-quotes . . . . . . . . . . . 24

IA.9 Comparison of the test of unbiasedness regressions between stocks with different levels of high-speed traders participation . . . . . . . . . . . 25 


\section{A. Best quotes during the pre-opening period}

The pre-opening quotes consist of bid and ask prices and their associated quantities. In the case of the TSE, the best bid and ask prices are determined differently during the pre-opening period than during the continuous trading period. During the continuous trading period, the best bid is the highest available bid price, and the best ask is the lowest available ask price. This means that the bid and ask schedules do not intersect as the submission of a buy order with a limit price greater or equal than the best available ask price will cause the immediate execution of that order and it will not join the queue in the limit order book.

On the contrary, during the pre-opening period no execution is allowed before the opening auction, when all orders are executed at a single price. Therefore, the best bid and ask prices reported during the pre-opening period are the respective prices at which the bid (demand) and ask (supply) schedules intersect. The best ask is identified as the smallest ask price at which the cumulative depth of the ask schedule is greater than the cumulative depth of the bid schedule. The best bid is identified as the largest bid price at which the cumulative depth of the bid schedule is greater than the cumulative depth of the ask schedule. ${ }^{1}$

This appendix illustrates how the best bid price and the best ask price are determined during the pre-opening period. First of all, the TSE computes the cumulative amount of eligible buy and sell orders (depth) at each price. Usually, more buy orders are accumulated around lower prices and more sell orders are accumulated around higher prices so that there is a point at which the situation of "cumulative buy orders" being greater than "cumulative sells" turns into "cumulative buys" being less than or equal to "cumulative sells". The best bid is the highest bid price at which the cumulative bid depth is greater than the cumulative ask depth and the best ask is the lowest ask price at which the cumulative ask depth is greater than the cumulative bid depth.

Therefore, the best bid and ask prices reported during the pre-opening period are the respective prices at which the bid (demand) and ask (supply) schedules intersect. Either the best ask or the best bid price is the opening price, as a result of the single price auction explained in Section III. In the pre-opening period, however, the cumulative amounts of buy and sell orders can be the same, particularly at the beginning of the pre-opening period when just a few orders have been entered. In these special situations, the TSE has another rule for determining the best bid and ask in the pre-opening period, which is based on yesterday's closing price or the upper or lower daily limit on the price of a stock. Refer to TSE (2015) for details.

\footnotetext{
${ }^{1}$ We use two different sources for the best bid and ask prices in the pre-opening period. First, we use the Thomson Reuters Tick History (TRTH) data with a millisecond time stamp. However, there is a time stamp mismatch between the order flow data provided by the TSE and the TRTH best quotes time stamp. Therefore, for the analysis that requires exact matching between these two databases, we construct the best bid-offer ourselves on a tick-by-tick basis. This is a non-trivial task due to the multiple rules employed by the TSE. We verify the sequence of our best bid and ask estimates using the TRTH database, and ensure that our estimates are consistent with the TRTH best bid and ask prices time stamped without a time delay.
} 


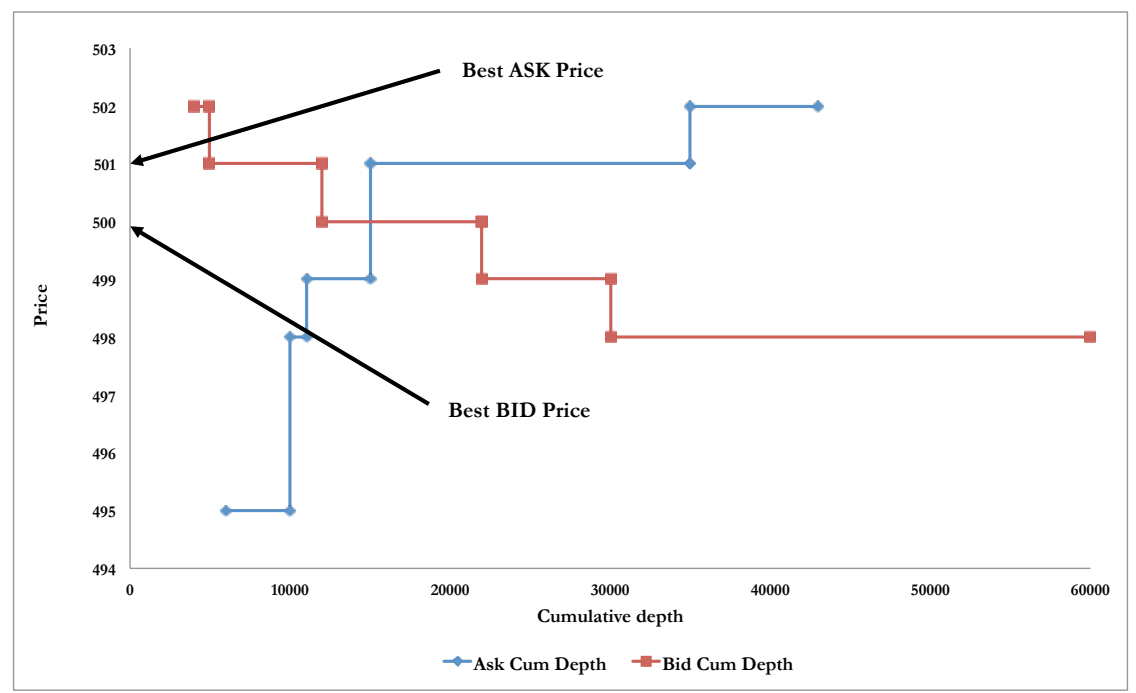

Figure IA.1. Determination of best bid and ask prices during the pre-opening period. This figure shows a hypothetical example of how the best bid price and the best ask price are determined during the pre-opening period. We plot bid (demand) and ask (supply) schedules with cumulative volume on the $X$-axis and price on the $Y$-axis. The blue line represents the ask schedule, while the red line represents the bid schedule. The best bid is the highest bid price at which the cumulative bid depth is greater than the cumulative ask depth. The best ask is the lowest ask price at which the cumulative ask depth is greater than the cumulative bid depth. 


\section{B. Configuration of multiple virtual servers (VSs) used by one trader}

VSs are used to send/receive data to/from the TSE and are the logical path established between TSE's system (matching engine) and a user's system in order to communicate with each other. There are 5,580 VSs in existence during our sample period. Most of them $(2,692)$ are used as single sVS and the rest as part of multiple-VS configurations. When using multiple VSs, each trader optimizes the configuration of VSs so that she can maximize the performance of her trading activity. Some traders trade a specific group of stocks every day, in which case they may fix the allocation of stocks to each VS. Other traders may change part of their allocation on a day-by-day basis. As Table I shows, by optimizing the number of stocks per VS, a trader can increase her speed significantly. Figure IA.2 illustrates one example of a VS configuration.

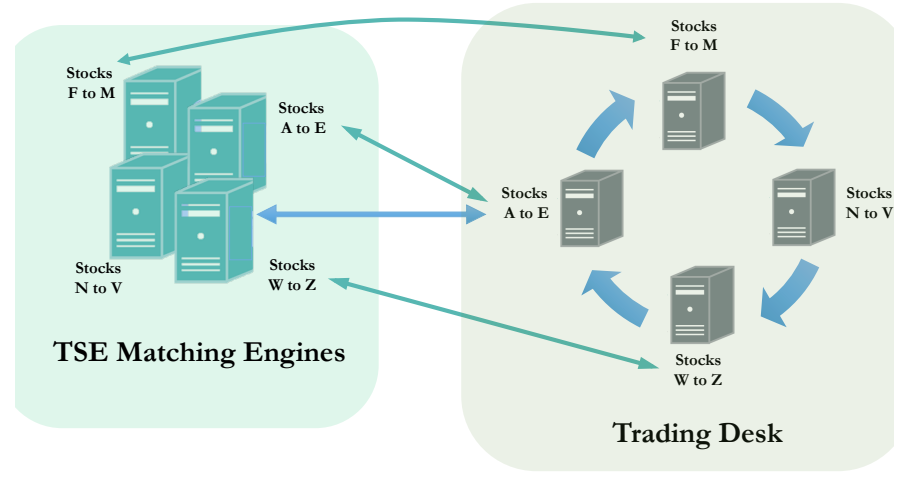

Figure IA.2. Illustration of a possible VS configuration. This figure displays a possible VS configuration for mimicking the TSE's matching engine. 


\section{Speed model estimation}

Due to the limitation on the number of quotes per second per VS imposed by the TSE (see footnote 17 of the paper), the coverage of stocks and intensity of quotes of a trader determine the size of their operation. Our novel data on virtual server IDs allow us to estimate the relation between speed, VS configuration, and quote intensity, using the following equation:

$$
\begin{aligned}
& \text { Speed }_{j, k, l}=a+b \ln \left(\text { Quote }_{j, k, l}\right)+ \\
& c \ln \left(\text { stock }_{k, l} / N V S_{l}\right)+d \ln \left(\text { MaxQuote }_{k, l}\right)+\epsilon_{j, k, l}
\end{aligned}
$$

Speed $_{j, k, l}$ is the speed measure for stock $j$, day $k$, and trader $l$. Quote ${ }_{j, k, l}$ is the number of quotes for stock $j$, day $k$, and trader $l$. Nstock $k, l$ is the number of stocks traded on day $k$ by trader $l$. $N V S_{l}$ is the number of virtual servers used by trader $l$ (a fixed number during our sample period). MaxQuote $_{k, l}$ is the maximum number of quotes per second sent by trader $l$ on day $k$.

The daily number of stocks per VS indicates the trader's speed requirement. The number of quotes is used by other studies to identify HFTs that engage in market making. The maximum number of quotes per second is another aspect of trading style; for example, an index arbitrager might execute a basket of 225 Nikkei Index constituents simultaneously. Our empirical measure of speed is limited by the time stamp unit of one millisecond, meaning that the distribution of observed elapsed time is clustered at one millisecond. Taking into account the censored nature of the dependent variable, we use a Tobit model to estimate equation (IA.1).

Table IA.1 shows a strong relation between the number of stocks per VS, the total number of quotes, and the maximum number of quotes per second. The smaller the number of stocks per VS, and the larger the number of quotes (maximum number of quotes per second), the lower is the speed. 


\section{Table IA.1 Speed model estimation}

This table shows estimation results, using Tobit regression, of the model, see equation (IA.1). Speed $d_{j, k, l}$ is the speed measure for stock $j$, day $k$, and trader $l$. Quote $e_{j, k, l}$ is the number of quotes for stock $j$, day $k$, and trader $l . N s_{\text {stock }}$, is the number of stocks traded on day $k$ by trader $l . N V S_{l}$ is the number of virtual servers used by trader $l$ (a fixed number during our sample period). MaxQuote $e_{k, l}$ is the maximum number of quotes per second sent by trader $l$ on day $k$. Our sample consists of 97 stocks from TOPIX100 during April and May 2013. Order flow data, with order IDs as well as virtual server IDs, are provided by the Tokyo Stock Exchange.

\begin{tabular}{lcc}
\hline \multicolumn{3}{c}{ Dependent variable: Speed $_{j, t, k}$} \\
\hline & Coef & \multicolumn{1}{c}{$t$-stat } \\
\hline Constant & 5.44 & 571.65 \\
$\ln \left(\right.$ Quot $\left._{j, t, k}\right)$ & -2.08 & -1555.60 \\
$\ln \left(\right.$ Nstock $\left._{t, k} / N V S_{k}\right)$ & 0.41 & 263.60 \\
$\ln \left(\right.$ MaxQuote $\left._{t, k}\right)$ & -1.35 & -489.31 \\
& & \\
Left-censored obs & \multicolumn{2}{c}{73,011} \\
Right-censored obs & \multicolumn{2}{c}{0} \\
Uncensored obs & $3,120,836$ \\
Total obs & $3,193,847$ \\
\hline
\end{tabular}




\section{An alternative classification scheme: Kirilenko et al. (2016)}

We applied to our data the classification scheme proposed by Brogaard, Hagströmer, Norden, and Riordan (2015), very similar to the Kirilenko, Kyle, Samadi, and Tuzun (2016) approach. This scheme splits the traders in two groups, HFTs and non-HFTs, according to a set of characteristics. In particular, a trader is defined as an HFT in a particular stock if and only if, on at least $50 \%$ of the active days, she satisfies the following criteria: First, her end-of-day inventory is no greater than $10 \%$ of her trading volume, for that stock, on that day. Second, her inventory at the end of each minute is no greater than $15 \%$ of her trading volume for that stock on that day. Third, her trading volume in that stock, on that day, is in the top quartile of the total trading volume for all traders in that stock on that day. ${ }^{2}$ This classification scheme is applied to only one month, April 2013, as there was a change in the virtual server ID definition at the beginning of May 2013. Table IA.2 presents a summary of trader characteristics based on this classification scheme. Some stylized facts: the median speed for HFTs is 2 milliseconds, compared to 4 milliseconds for FAST/SMALL traders. HFTs are characterized by a $0.95 \%$ end-of-day inventory, in contrast to the $15.20 \%$ end-ofday inventory of our FAST/SMALL traders. Note that HFTs also have a lower intraday inventory and lower volatility of intraday inventory than FAST/SMALL traders. The median number of times that the inventory crosses zero for HFTs equals 14 times per day, while the number is just twice per day for FAST/SMALL traders.

\footnotetext{
${ }^{2}$ Brogaard, Hagströmer, Norden, and Riordan (2015) use top quartile of proprietary trading volume rather than aggregate trading volume as we do. Unfortunately, our data does not allow us to distinguish between proprietary and client trading volume.
} 


\section{Table IA.2 Classification scheme proposed by Kirilenko et al. (2016)}

This table shows summary statistics for the classification of traders based on Kirilenko, Kyle, Samadi, and Tuzun (2016). In this case, we divide traders into two groups (HFTs and non-HFTs) using information from the continuous trading session of the same day. Panel A shows the median number of traders, the median of the $1 \%, 10 \%$, and $50 \%$ percentiles of the trader-stock-day speed distribution, and the median minimum speed. Panel B shows the median number of traders, median end-of-day inventory, median average intraday inventory and its volatility, and the median number of times during the day that the inventory crosses zero, per trader-stock-day. These characteristics are presented per group for the period of April 2013, for the 97 stocks from TOPIX100. Order flow data, with order IDs as well as virtual server IDs, are provided by the Tokyo Stock Exchange.

\begin{tabular}{|c|c|c|c|c|c|}
\hline \multicolumn{6}{|c|}{ Panel A: Speed of traders } \\
\hline & & & Median of & & \\
\hline & \# of traders & Min Speed & P1 Speed & P5 Speed & P10 Speed \\
\hline $\mathrm{HFT}$ & 5 & 0.002 & 0.002 & 0.008 & 0.020 \\
\hline Non-HFT & 905 & 1.917 & 1.973 & 4.102 & 9.165 \\
\hline \multicolumn{6}{|c|}{ Panel B: Inventory of traders } \\
\hline & \multicolumn{5}{|c|}{ Median of } \\
\hline & \# of traders & $\begin{array}{l}\text { End-of-day } \\
\text { Inventory }\end{array}$ & $\begin{array}{l}\text { Intraday } \\
\text { inventory }\end{array}$ & $\begin{array}{c}\text { Volatility } \\
\text { of intraday } \\
\text { inventory }\end{array}$ & $\begin{array}{l}\text { \# of zero } \\
\text { crossings }\end{array}$ \\
\hline $\mathrm{HFT}$ & 5 & $0.95 \%$ & $2.91 \%$ & $2.27 \%$ & 14 \\
\hline Non-HFT & 905 & $100.00 \%$ & $53.89 \%$ & $21.60 \%$ & 0 \\
\hline
\end{tabular}

Table IA.3 shows how the two classification schemes compare to one another. In particular, traders classified as HFTs under the Kirilenko, Kyle, Samadi, and Tuzun (2016) scheme are most likely to fall into either the FAST/SMALL or the MODERATE/SMALL group. 


\section{Table IA.3 Comparison of classifications}

This table shows the summary comparison of the classification of traders proposed in this paper versus that based on Kirilenko, Kyle, Samadi, and Tuzun (2016) for 97 stocks from TOPIX100 during April 2013. The classification proposed in this paper splits traders into 12 groups on a stock-day basis, as reported in Table II. The classification of traders based on Kirilenko, Kyle, Samadi, and Tuzun (2016) splits traders into two groups (HFTs and non-HFTs). Order flow data, with order IDs as well as virtual server IDs, are provided by the Tokyo Stock Exchange.

\begin{tabular}{llcc}
\hline Speed & Inventory & HFT & Non-HFT \\
\hline \multirow{4}{*}{ FAST } & LARGE & 10 & 124,237 \\
& MEDIUM & 66 & 134,622 \\
& SMALL (HFTs) & 7,701 & 130,443 \\
& NOTRADE & 0 & 68,333 \\
\multirow{2}{*}{ MODERATE } & LARGE & 1 & 153,047 \\
& SMAIUM & 26 & 118,539 \\
& NOTRADE & 1,380 & 110,629 \\
& LARGE & 5 & 79,764 \\
\multirow{5}{*}{ SLOW } & MEDIUM & 12 & 336,510 \\
& SMALL & 0 & 64,533 \\
& NOTRADE & 15 & 53,209 \\
& & 4 & 66,724 \\
\hline
\end{tabular}




\section{E. Order flow in the pre-opening period}

As explained in Section III of the paper, the pre-opening period of the TSE starts at 8 am. All member firms begin to send orders from their customers' and their own accounts to the exchange. The results from the three different trading-speed groups are reported in Figure IA.3, Panel A. The green line represents orders from FAST traders, who play a dominant role during the whole pre-opening period. FAST traders submit $50.5 \%$ of the total number of orders in the pre-opening period, with MODERATE and SLOW traders submitting $42.5 \%$ and $7.0 \%$, respectively. In the first 10 minutes of the pre-opening period, $73.7 \%$ of the total number of orders of the entire preopening period are submitted. FAST traders submit $36.0 \%$ out of their $50.5 \%$ of orders in the first 10 minutes, MODERATE and SLOW traders submit $32.8 \%$ and $5.0 \%$, respectively. The order submission intensity slows down after the first 10 minutes, and is reactivated 10 minutes before the official opening time. The high level of order submissions in the first 10 minutes partly reflects the accumulation of orders overnight. Early investors also have a desire to lead price formation for the opening call auction.

Figure IA.3, Panel B, shows the order flow for the four different inventory groups. The majority of the order flow comes from trades with small and medium inventories. Figure IA.3, Panels C, D, and $\mathrm{E}$ present the pattern of order submission activity for the FAST, MODERATE, and SLOW traders during the pre-opening period, classified according to level of inventory for each group. They clearly show a peak at the very beginning of the period for traders with SMALL and MEDIUM levels of inventory, and another very close to the opening time for FAST/SMALL (HFTs) traders, vastly exceeding the number of orders submitted by slower traders. FAST traders submit $7.5 \%$ of the total number of orders in the last 10 minutes of the pre-opening period, and MODERATE and SLOW traders submit $4.4 \%$ and $1.0 \%$, respectively. Traders with a LARGE inventory and those in the NOTRADE group submit most of their orders at $8 \mathrm{am}$. One of the reasons traders submit more orders at 8 am is to ensure a higher probability of execution of their orders due to the time-priority-based allocation most brokers employ, as explained in Section III.

Figure IA.4 Panel A shows the new order submissions and cancellations as a percentage of all orders submitted by FAST, MODERATE, and SLOW traders, in the last 10 minutes of the pre-opening period. While the magnitude of the order submission differs (as the scale of the $y$-axis differs between FAST, MODERATE, and SLOW traders), the pattern is quite similar for all three groups. Traders accentuate their pattern of order submission during the last three minutes of the pre-opening period. A rise in order cancellations (indicated by the black line) happens suddenly, one second before $9 \mathrm{am}$, for all trader groups. For instance, the percentage of cancellation messages increases from less than $0.1 \%$ to $0.9 \%$ (of the total number of orders in the pre-opening period) per second for FAST traders, and from less than $0.01 \%$ to around $0.25 \%$ for MODERATE-speed traders.

Figure IA.4 Panel B depicts order submissions and cancellations for the different inventory subgroups within the FAST group. It is interesting to note that cancellations from all trader groups reach their peak at the very last second. We investigate this in detail at the millisecond level and present the results in Figure IA.5 Panel A.

We confirm that the cancellations indeed occur less than one second before $9 \mathrm{am}$. As Figure IA.5 Panel B shows, the cancellation phenomenon starts at 500 milliseconds before 9 am and peaks at 130 milliseconds before $9 \mathrm{am}$. The peak is particularly pronounced for FAST traders and is not specifically related to inventory. The final action of limit price adjustment takes place just milliseconds before the opening time, which would not be possible in the absence of a low-latency trading environment. 

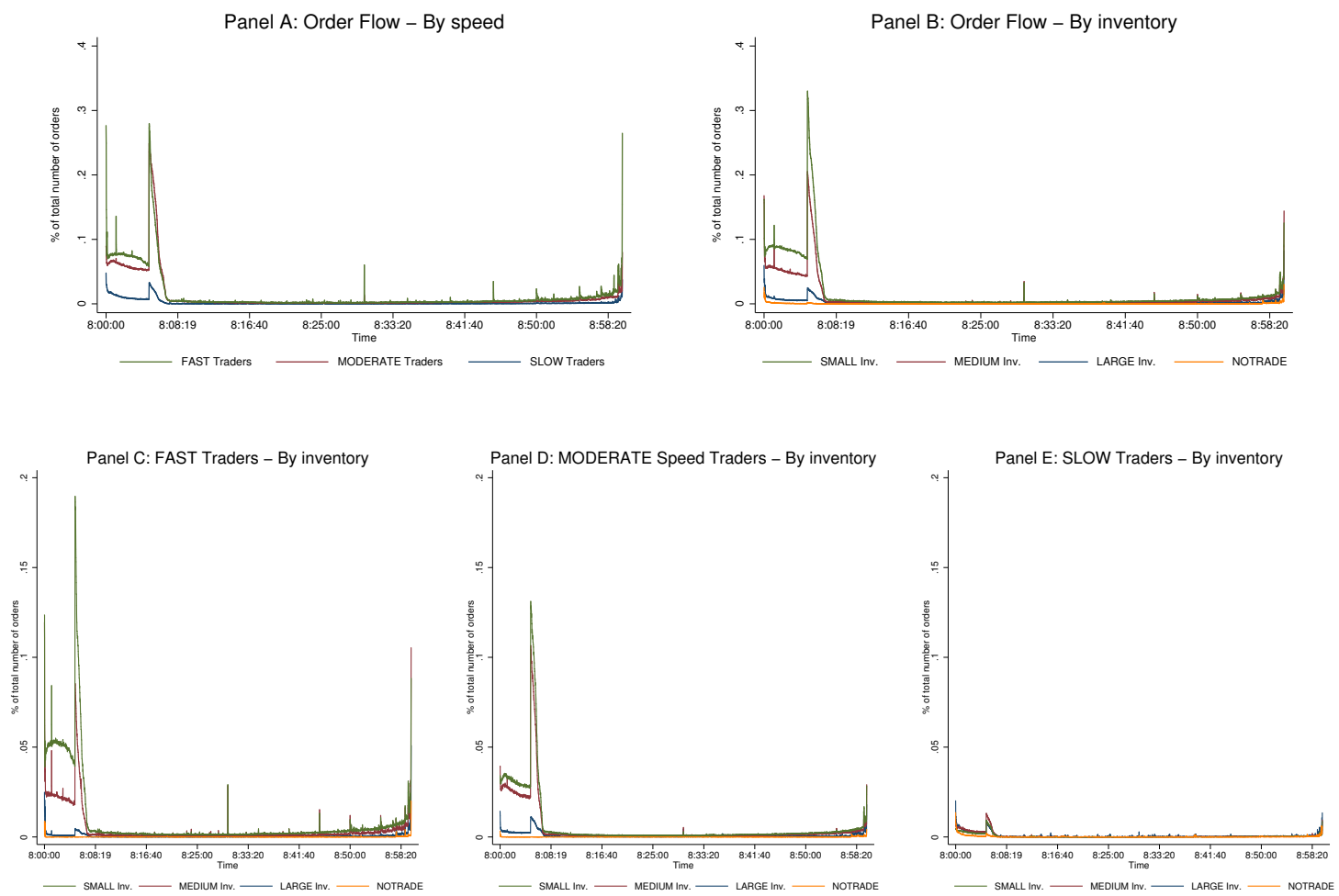

Fig. IA.3. Flow of total order submission in the pre-opening period This figure depicts the second-bysecond order flow for 97 stocks from the TOPIX100 during the sample period of April and May 2013. The Tokyo Stock Exchange starts receiving orders at 8 am and starts the call auction at $9 \mathrm{am}$. The average percentage of the total number of orders is the total number of orders in each second divided by the total number of orders submitted during the whole pre-opening period (8:00:00.000 - 8:59:59.999). The $Y$-axis represents the percentage of the total number of new orders in the pre-opening period, and the $X$-axis represents the time in seconds between 8 am and 9 am. Panel A depicts the average percentage of the total number of orders by speed group, as defined in Table II using information about speed and inventory from the same day's continuous session, per second. Panels B, C, and D report, for each speed group, the average percentage of the total number of orders according to level of inventory, as defined in Table II. Order flow data, with order IDs as well as virtual server IDs, are provided by the Tokyo Stock Exchange. 
Panel A - Submission of new orders and cancellations by speed category

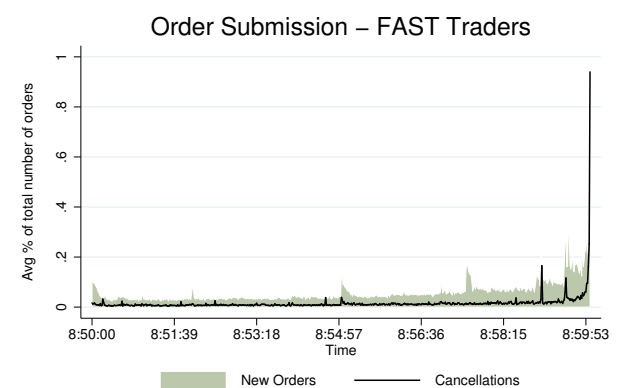

Order Submission - MODERATE Speed Traders
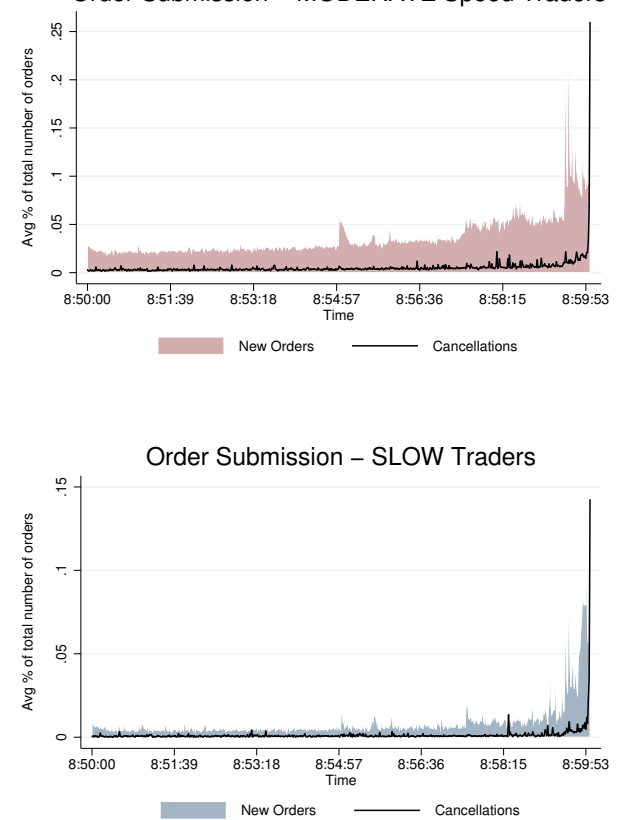

Panel B - Submission of new orders and cancellations by FAST traders
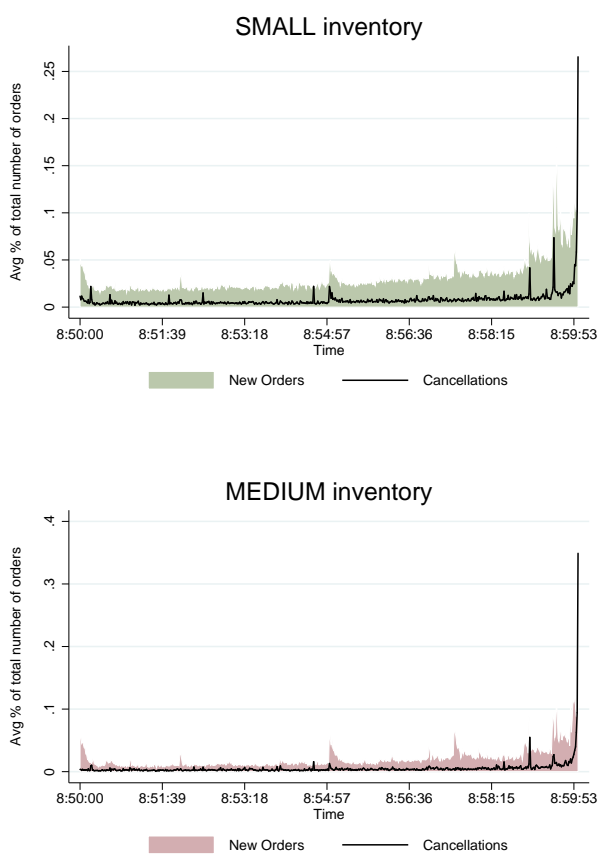

LARGE inventory

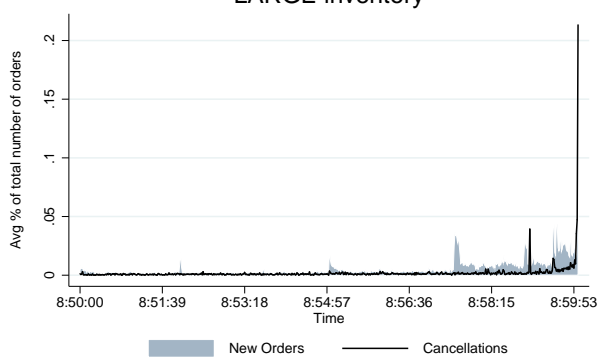

Figure IA.4. Flow of new orders and cancellations in the last 10 minutes of the pre-opening period The figure depicts the second-by-second new orders and cancellations for 97 stocks from the TOPIX100 during the sample period of April and May 2013. The Tokyo Stock Exchange starts receiving orders at 8 am and starts the call auction at $9 \mathrm{am}$. New orders and cancellations are measured as percentage of the total number of orders submitted in the last 10 minutes of the pre-opening period (8:50:00.000 - 8:59:59.999). The $Y$-axis represents the percentage of the total number of orders in the last 10 minutes of the pre-opening period, and the $X$-axis represents the time in seconds between 8:50 am and $9 \mathrm{am}$. Panel A depicts the average percentage of the total number of orders by speed group, as defined in Table II using information about speed and inventory from the same day's continuous session, during the sample period, per second. Panel B reports, for FAST traders, new orders and cancellations, according to the level of inventory. Order flow data, with order IDs as well as virtual server IDs, are provided by the Tokyo Stock Exchange. 
Panel A - Order flow for all traders

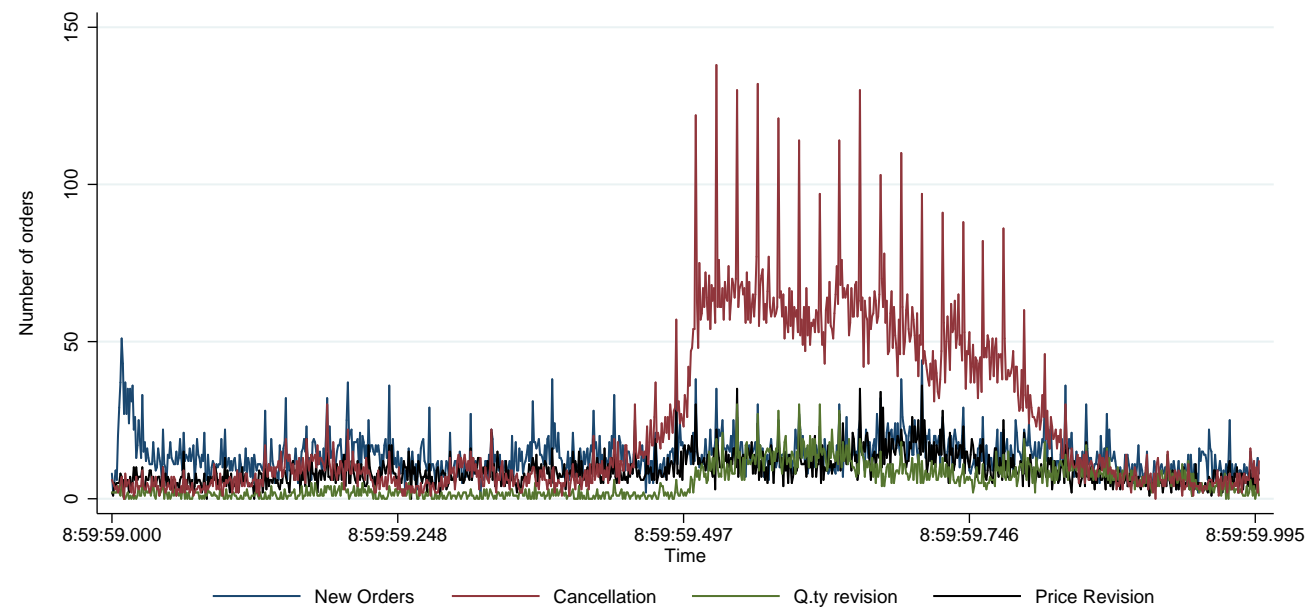

Panel B - Cancellations by speed and inventory group
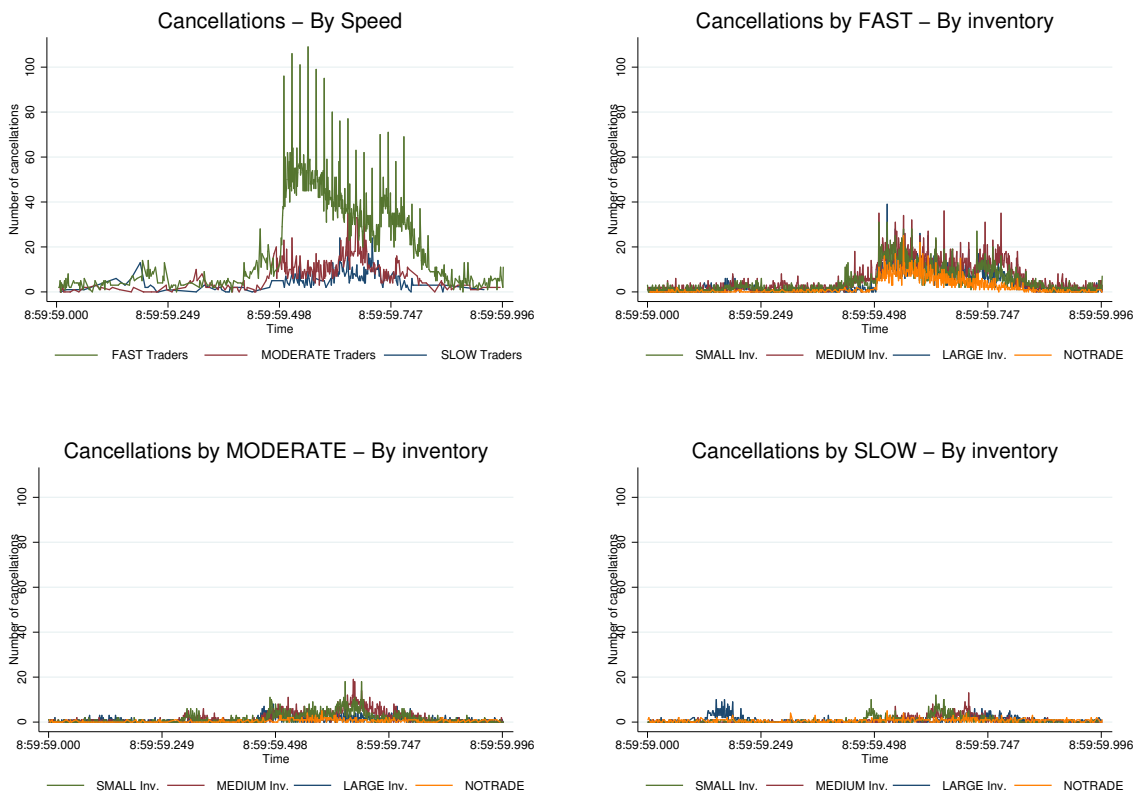

Figure IA.5. Order flow during the last second of the pre-opening period shows four types of order submission activity: new orders (blue line), cancellations (red line), quantity revisions (green line), and price revisions (black line), in the last second of the pre-opening period (8:59:59.000 - 8:59:59.999) at the millisecond level, for all 97 stocks from the TOPIX100 during the sample period of April and May 2013. Panel A reports traders' order submission activity. Panel B focuses on cancellations, distinguishing between the different groups of traders. Order flow data, with order IDs as well as virtual server IDs, are provided by the Tokyo Stock Exchange. 


\section{F. Aggressive orders}

Among the orders submitted during the pre-opening period, we can identify those orders with the potential to impact the prevailing quotes. We call them "aggressive orders" (as in Biais, Hillion, and Spatt (1995), Ranaldo (2004), Duong, Kalev, and Krishnamurti (2009), and Yamamoto (2011)). The TSE uses unique rules for determining the best pre-opening bid and ask quotes. These rules are different from those applied in the continuous trading period and are briefly explained in Section III. There are four cases of orders that we categorize as aggressive: first, all market orders; second, a limit buy order with a limit price greater than or equal to the prevailing best bid; third, a limit sell order with a limit price less than or equal to the prevailing ask; fourth, any orders submitted at a time when the best bid equals the best ask. ${ }^{3}$

When an order that satisfies one of the abovementioned conditions is newly entered, modified, or cancelled, it has the potential to impact the prevailing quotes. Table IA.4 shows the total number of orders from the 12 trader groups defined earlier. The largest proportion of aggressive orders comes from Active-w-Trade FAST/SMALL traders (HFTs). On average, they submit 128.24 aggressive orders (46.30 market orders and 80.21 limit orders). The next largest group of aggressive traders are the FAST/MEDIUM traders, who submit 85.79 aggressive orders (39.57 market orders and 44.76 limit orders). Note that our classification does not take into account trading share, such as top quartile of volume, and that only one quarter of FAST/SMALL traders (HFTs) participate in the pre-opening period but their submission of aggressive orders is significantly greater than that of the other groups. The ratios of aggressive limit orders to the total number of limit orders from these two most aggressive groups of traders are $27.82 \%$ and $31.80 \%$, respectively. FAST/SMALL traders (HFTs) place the highest number of aggressive limit orders in relation to the total number of orders, which indicates their interest in affecting the price.

\footnotetext{
${ }^{3}$ Such a situation occurs when the cumulative amount of buy orders equals that of sell orders. Thus, the next order must cause an imbalance between buy and sell orders and make the best ask higher than the best bid price. We refer to such orders as "locked orders." Cao, Ghysels, and Hatheway (2000) analyze locked/crossed market quotes during the NASDAQ pre-opening period. In the TSE's pre-opening period, market best quotes may be locked, which means that the best ask equals the best bid, but crossed quotes (which means that the best bid is greater than the best ask) never happen, by rule.
} 


\section{Table IA.4 Aggressive orders during pre-opening period}

This table shows summary statistics across stock-days for order aggressiveness for the 12 trader groups, during the pre-opening period excluding the first 10 minutes, for 97 stocks from the TOPIX100, during the sample period of April and May 2013. Order flow data, with order IDs as well as virtual server IDs, are provided by the Tokyo Stock Exchange.

\begin{tabular}{|c|c|c|c|c|c|c|c|}
\hline \multicolumn{8}{|c|}{ Panel A: Active-w/o-Trade } \\
\hline Speed & Inventory & $\begin{array}{l}\text { Total \# of } \\
\text { aggressive } \\
\text { orders }\end{array}$ & $\begin{array}{c}\text { \# of market } \\
\text { orders }\end{array}$ & $\begin{array}{c}\text { \# of aggressive } \\
\text { limit orders }\end{array}$ & $\begin{array}{l}\# \text { of zero } \\
\text { imabalance } \\
\text { orders }\end{array}$ & $\begin{array}{l}\text { Ratio of total } \\
\text { order } \\
\text { aggressiveness }\end{array}$ & $\begin{array}{c}\text { Ratio of limit } \\
\text { order } \\
\text { aggressiveness }\end{array}$ \\
\hline FAST & $\begin{array}{l}\text { LARGE } \\
\text { MEDIUM } \\
\text { SMALL (HFTs) } \\
\text { NOTRADE }\end{array}$ & $\begin{array}{l}13.20 \\
17.75 \\
28.01 \\
2.83\end{array}$ & $\begin{array}{l}0.41 \\
0.90 \\
0.51 \\
0.07\end{array}$ & $\begin{array}{c}12.03 \\
16.24 \\
26.47 \\
2.50\end{array}$ & $\begin{array}{l}0.76 \\
0.61 \\
1.02 \\
0.26\end{array}$ & $\begin{array}{l}46.46 \% \\
46.46 \% \\
47.92 \% \\
35.14 \%\end{array}$ & $\begin{array}{l}44.16 \% \\
44.26 \% \\
46.52 \% \\
32.39 \%\end{array}$ \\
\hline MODERATE & $\begin{array}{l}\text { LARGE } \\
\text { MEDIUM } \\
\text { SMALL } \\
\text { NOTRADE }\end{array}$ & $\begin{array}{l}5.53 \\
8.53 \\
8.89 \\
1.41\end{array}$ & $\begin{array}{l}0.65 \\
0.87 \\
0.73 \\
0.17\end{array}$ & $\begin{array}{l}4.11 \\
6.58 \\
7.22 \\
1.01\end{array}$ & $\begin{array}{l}0.77 \\
1.08 \\
0.95 \\
0.23\end{array}$ & $\begin{array}{l}27.76 \% \\
25.85 \% \\
28.37 \% \\
26.43 \%\end{array}$ & $\begin{array}{l}22.20 \% \\
21.19 \% \\
24.32 \% \\
20.43 \%\end{array}$ \\
\hline SLOW & $\begin{array}{l}\text { LARGE } \\
\text { MEDIUM } \\
\text { SMALL } \\
\text { NOTRADE } \\
\end{array}$ & $\begin{array}{l}4.61 \\
2.39 \\
2.10 \\
4.03 \\
\end{array}$ & $\begin{array}{l}0.49 \\
0.22 \\
0.16 \\
0.50\end{array}$ & $\begin{array}{l}3.45 \\
1.78 \\
1.68 \\
2.99\end{array}$ & $\begin{array}{l}0.67 \\
0.39 \\
0.26 \\
0.53\end{array}$ & $\begin{array}{l}27.86 \% \\
25.89 \% \\
28.35 \% \\
27.74 \%\end{array}$ & $\begin{array}{l}22.42 \% \\
20.67 \% \\
24.02 \% \\
22.21 \%\end{array}$ \\
\hline \multicolumn{8}{|c|}{ Panel B: Active-w-Trade } \\
\hline Speed & Inventory & $\begin{array}{l}\text { Total \# of } \\
\text { aggressive } \\
\text { orders }\end{array}$ & $\begin{array}{c}\text { \# of market } \\
\text { orders }\end{array}$ & $\begin{array}{c}\text { \# of aggressive } \\
\text { limit orders }\end{array}$ & $\begin{array}{l}\# \text { of zero } \\
\text { imabalance } \\
\text { orders }\end{array}$ & $\begin{array}{l}\text { Ratio of total } \\
\text { order } \\
\text { aggressiveness }\end{array}$ & $\begin{array}{c}\text { Ratio of limit } \\
\text { order } \\
\text { aggressiveness }\end{array}$ \\
\hline FAST & $\begin{array}{l}\text { LARGE } \\
\text { MEDIUM } \\
\text { SMALL (HFTs) }\end{array}$ & $\begin{array}{c}13.51 \\
85.79 \\
128.24\end{array}$ & $\begin{array}{c}6.52 \\
39.57 \\
46.30\end{array}$ & $\begin{array}{c}6.82 \\
44.76 \\
80.21\end{array}$ & $\begin{array}{l}0.17 \\
1.46 \\
1.73\end{array}$ & $\begin{array}{l}60.23 \% \\
47.20 \% \\
38.13 \%\end{array}$ & $\begin{array}{l}43.33 \% \\
31.80 \% \\
27.82 \%\end{array}$ \\
\hline MODERATE & $\begin{array}{l}\text { LARGE } \\
\text { MEDIUM } \\
\text { SMALL }\end{array}$ & $\begin{array}{l}12.28 \\
54.43 \\
77.02\end{array}$ & $\begin{array}{c}6.00 \\
22.02 \\
29.07\end{array}$ & $\begin{array}{c}6.04 \\
30.75 \\
45.83\end{array}$ & $\begin{array}{l}0.24 \\
1.66 \\
2.12\end{array}$ & $\begin{array}{l}51.24 \% \\
39.51 \% \\
37.44 \%\end{array}$ & $\begin{array}{l}34.08 \% \\
26.96 \% \\
26.26 \%\end{array}$ \\
\hline SLOW & $\begin{array}{l}\text { LARGE } \\
\text { MEDIUM } \\
\text { SMALL }\end{array}$ & $\begin{array}{c}14.37 \\
8.79 \\
6.40 \\
\end{array}$ & $\begin{array}{l}8.24 \\
4.41 \\
3.09\end{array}$ & $\begin{array}{l}5.90 \\
4.08 \\
3.10\end{array}$ & $\begin{array}{l}0.22 \\
0.31 \\
0.21\end{array}$ & $\begin{array}{l}74.99 \% \\
57.75 \% \\
58.13 \%\end{array}$ & $\begin{array}{l}55.18 \% \\
38.82 \% \\
40.23 \%\end{array}$ \\
\hline
\end{tabular}




\section{G. Additional analysis for price discovery}

\section{G.1. Regression on WPC per stock-day}

In the main paper we perform the test on whether the contribution from trader groups is significantly different by aggregating $W P C$ across stocks. As a robustness check, we verify whether results are the same if we avoid WPC aggregation across all the stocks. Results of the panel regression are reported in Table IA.5. The table shows that the results are qualitatively the same of those reported in Table VIII, Panel B, of the main paper.

\section{Table IA.5 Weighted price discovery regression by stock-date}

This table presents regression analysis by stock-date for the weighted price discovery contribution (WPC) during the pre-opening period and first 30-minutes of the continuous session (see equation (7)) for 97 stocks from the TOPIX100 during the sample period of April and May 2013. WPC is attributable to aggressive orders. We divide all traders into 12 groups on a stock-day basis, as described in Table II, using information about speed and inventory from the same day's continuous session. We also split traders into 3 categories: traders that are not active during pre-opening period (Non-Active), traders that participate during the preopening period, but do not trade at the opening call auction (Active-w/o-trade), and traders that participate during the pre-opening period and trade at the opening call auction (Active-w-trade). ${ }^{* * *},{ }^{* *},{ }^{*}$ correspond to $1 \%, 5 \%$, and $10 \%$ significance levels. Order flow data, with order IDs as well as virtual server IDs, are provided by the Tokyo Stock Exchange.

\begin{tabular}{|c|c|c|c|c|c|c|c|c|}
\hline \multirow[b]{2}{*}{ Speed } & \multirow[b]{2}{*}{ Inventory } & \multicolumn{3}{|c|}{ Pre-opening period } & \multicolumn{4}{|c|}{ First 30-minutes of the continuous session } \\
\hline & & Active-w/o-Trade & Active-w-Trade & Total & Active-w/o-Trade & Active-w-Trade & Non-Active & Total \\
\hline FAST & $\begin{array}{l}\text { LARGE } \\
\text { MEDIUM } \\
\text { SMALL (HFTs) } \\
\text { NOTRADE }\end{array}$ & $\begin{array}{c}-0.02^{* * *} \\
-0.03^{* * *} \\
-0.02^{* * *} \\
0.01^{*}\end{array}$ & $\begin{array}{l}-0.04^{* * *} \\
-0.28^{* * *} \\
-0.16^{* * *}\end{array}$ & $\begin{array}{c}-0.02^{* * *} \\
-0.14^{* * *} \\
-0.08^{* * *} \\
0.02^{* * *}\end{array}$ & $\begin{array}{c}-0.01 \\
-0.06^{* * *} \\
-0.02\end{array}$ & $\begin{array}{c}-0.02 \\
-0.14^{*} \\
0.05\end{array}$ & $\begin{array}{c}-0.07 \\
-0.23^{* * *} \\
-0.49^{* * *}\end{array}$ & $\begin{array}{c}-0.02 \\
-0.13^{* * *} \\
-0.14^{* * *}\end{array}$ \\
\hline MODERATE & $\begin{array}{l}\text { LARGE } \\
\text { MEDIUM } \\
\text { SMALL } \\
\text { NOTRADE }\end{array}$ & $\begin{array}{c}-0.01 \\
-0.02^{* * *} \\
-0.02^{* * *} \\
0.01^{*}\end{array}$ & $\begin{array}{l}-0.04^{* * *} \\
-0.11^{* * *} \\
-0.13^{* * *}\end{array}$ & $\begin{array}{c}-0.01^{* *} \\
-0.05^{* * *} \\
-0.06^{* * *} \\
0.02^{* * *}\end{array}$ & $\begin{array}{c}0.02^{*} \\
0.02 \\
-0.02\end{array}$ & $\begin{array}{c}0.01 \\
0.10^{* * *} \\
-0.04\end{array}$ & $\begin{array}{c}-0.03^{*} \\
-0.11^{* * *} \\
-0.10^{* * *}\end{array}$ & $\begin{array}{c}0.02^{*} \\
0.02 \\
-0.04^{* *}\end{array}$ \\
\hline SLOW & $\begin{array}{l}\text { LARGE } \\
\text { MEDIUM } \\
\text { SMALL } \\
\text { NOTRADE } \\
\end{array}$ & $\begin{array}{c}-0.02^{* * *} \\
-0.00 \\
0.00 \\
-0.01^{* *} \\
\end{array}$ & $\begin{array}{l}-0.05^{* * *} \\
-0.03^{* * *}\end{array}$ & $\begin{array}{c}-0.02^{* * *} \\
-0.00 \\
0.02^{* * *}\end{array}$ & $\begin{array}{c}0.01 \\
-0.01 \\
-0.02^{*}\end{array}$ & $\begin{array}{l}0.00 \\
0.01\end{array}$ & $\begin{array}{c}-0.09^{* * *} \\
-0.02^{* *} \\
-0.03^{* * *}\end{array}$ & $\begin{array}{l}-0.01 \\
0.01^{*}\end{array}$ \\
\hline & $\begin{array}{l}\text { Constant } \\
\# \text { obs } \\
\text { Adj } R^{2} \\
\text { Clustered St.Err. }\end{array}$ & $\begin{array}{c}-0.01^{* * *} \\
66,885 \\
0.033 \\
\text { By Stock }\end{array}$ & & $\begin{array}{c}-0.03^{* * * *} \\
66,885 \\
0.015 \\
\text { By Stock }\end{array}$ & $\begin{array}{c}-0.00 \\
85,995 \\
0.003 \\
\text { By Stock }\end{array}$ & & & $\begin{array}{c}-0.02^{* * * *} \\
85,995 \\
0.001 \\
\text { By Stock }\end{array}$ \\
\hline
\end{tabular}

\section{G.2. Price discovery with different reference price}

In the main paper we use auction price as a reference price to investigate price discovery. However, as highlighted by Stoll and Whaley (1990); Amihud and Mendelson (1991); Berkman, Koch, Tuttle, and Zhang (2012), this price is highly volatile and therefore the analysis on price discovery could be biased because of this. Therefore, to check the robustness of our results, we repeat the $W P C$ analysis using as reference price the average price between 9:30 and 9:35 of the continuous section. Results are reported in Table IA.6 and confirm those obtained by using the auction price as reference price. 


\section{Table IA.6 Contribution to weighted price discovery: different reference prices}

This table presents the summary statistics for the weighted price discovery contribution $(W P C)$ during the pre-opening period for 97 stocks from the TOPIX100 during the sample period of April and May 2013 for different reference prices: opening price and price at 09:30. WPC is attributable to aggressive orders (see equation (7)). We divide all traders into 12 groups on a stock-day basis, as described in Table II, using information about speed and inventory from the same day's continuous session. We also split traders into 2 categories: traders that participate during the pre-opening period, but do not trade at the opening call auction (Active-w/o-trade), and traders that participate during the pre-opening period and trade at the opening call auction (Active-w-trade). Order flow data, with order IDs as well as virtual server IDs, are provided by the Tokyo Stock Exchange.

\begin{tabular}{|c|c|c|c|c|c|c|c|}
\hline \multirow[b]{2}{*}{ Speed } & \multirow[b]{2}{*}{ Inventory } & \multicolumn{3}{|c|}{ Reference price: opening price } & \multicolumn{3}{|c|}{ Reference price: price at 09:30 } \\
\hline & & Active-w/o-Trade & Actie-w-Trade & Total & Active-w/o-Trade & Active-w-Trade & Total \\
\hline FAST & $\begin{array}{l}\text { LARGE } \\
\text { MEDIUM } \\
\text { SMALL (HFTs) } \\
\text { NOTRADE }\end{array}$ & $\begin{array}{l}-3.01 \% \\
-3.55 \% \\
-2.56 \% \\
-0.50 \%\end{array}$ & $\begin{array}{c}-4.54 \% \\
-23.11 \% \\
-13.67 \%\end{array}$ & $\begin{array}{l}-7.55 \% \\
-26.66 \% \\
-16.23 \% \\
-0.50 \%\end{array}$ & $\begin{array}{l}-3.85 \% \\
-4.18 \% \\
-3.10 \% \\
-0.52 \%\end{array}$ & $\begin{array}{l}-4.54 \% \\
-25.24 \% \\
-11.00 \%\end{array}$ & $\begin{array}{c}-8.39 \% \\
-29.42 \% \\
-14.10 \% \\
-0.52 \%\end{array}$ \\
\hline MODERATE & $\begin{array}{l}\text { LARGE } \\
\text { MEDIUM } \\
\text { SMALL } \\
\text { NOTRADE }\end{array}$ & $\begin{array}{l}-1.68 \% \\
-2.95 \% \\
-2.40 \% \\
-0.42 \%\end{array}$ & $\begin{array}{l}-3.99 \% \\
-9.90 \% \\
-11.21 \%\end{array}$ & $\begin{array}{l}-5.67 \% \\
-12.85 \% \\
-13.61 \% \\
-0.42 \%\end{array}$ & $\begin{array}{l}-1.86 \% \\
-3.21 \% \\
-2.47 \% \\
-0.35 \%\end{array}$ & $\begin{array}{l}-2.45 \% \\
-7.24 \% \\
-11.66 \%\end{array}$ & $\begin{array}{c}-4.31 \% \\
-10.45 \% \\
-14.13 \% \\
-0.35 \%\end{array}$ \\
\hline SLOW & $\begin{array}{l}\text { LARGE } \\
\text { MEDIUM } \\
\text { SMALL } \\
\text { NOTRADE }\end{array}$ & $\begin{array}{l}-2.55 \% \\
-1.32 \% \\
-0.89 \% \\
-2.20 \%\end{array}$ & $\begin{array}{l}-5.12 \% \\
-3.31 \% \\
-1.12 \%\end{array}$ & $\begin{array}{l}-7.67 \% \\
-4.63 \% \\
-2.01 \% \\
-2.20 \%\end{array}$ & $\begin{array}{l}-2.75 \% \\
-1.46 \% \\
-1.46 \% \\
-2.47 \%\end{array}$ & $\begin{array}{l}-5.70 \% \\
-3.60 \% \\
-0.90 \%\end{array}$ & $\begin{array}{l}-8.45 \% \\
-5.06 \% \\
-2.36 \% \\
-2.47 \%\end{array}$ \\
\hline
\end{tabular}




\section{G.3. Price discovery by order type}

Our data allow us to measure $P D C$ by individual order, so that we can aggregate $W P C$ according to the trader group that submitted the order, and show the proportion of the price contribution made by a particular trader group and order type (similarly to Barclay and Warner (1993) and Chakravarty (2001)). Which types of orders contribute most to price discovery? According to Table IA.7, the types of orders contributing most to the $W P C$ are new limit and market orders submitted by Active-w-Trade traders. Cancellations of market orders and price revisions of limit orders also contribute to price discovery. On the other hand, quantity revisions and cancellations of limit orders increase the deviation of the quoted price from the opening price. Orders from Active$\mathrm{w} / \mathrm{o}$-Trade traders have a marginal effect. Our overall results indicate that quote setting during the pre-opening period is conducted by the Active-w-Trade FAST/SMALL (HFTs), FAST/MEDIUM, MODERATE/SMALL, and MODERATE/MEDIUM groups. Therefore, traders with high speeds and small inventories are indeed the ones that contribute the most to price discovery during the pre-opening period, even though there is no trading in this period, and only a fraction of fast traders participate. 


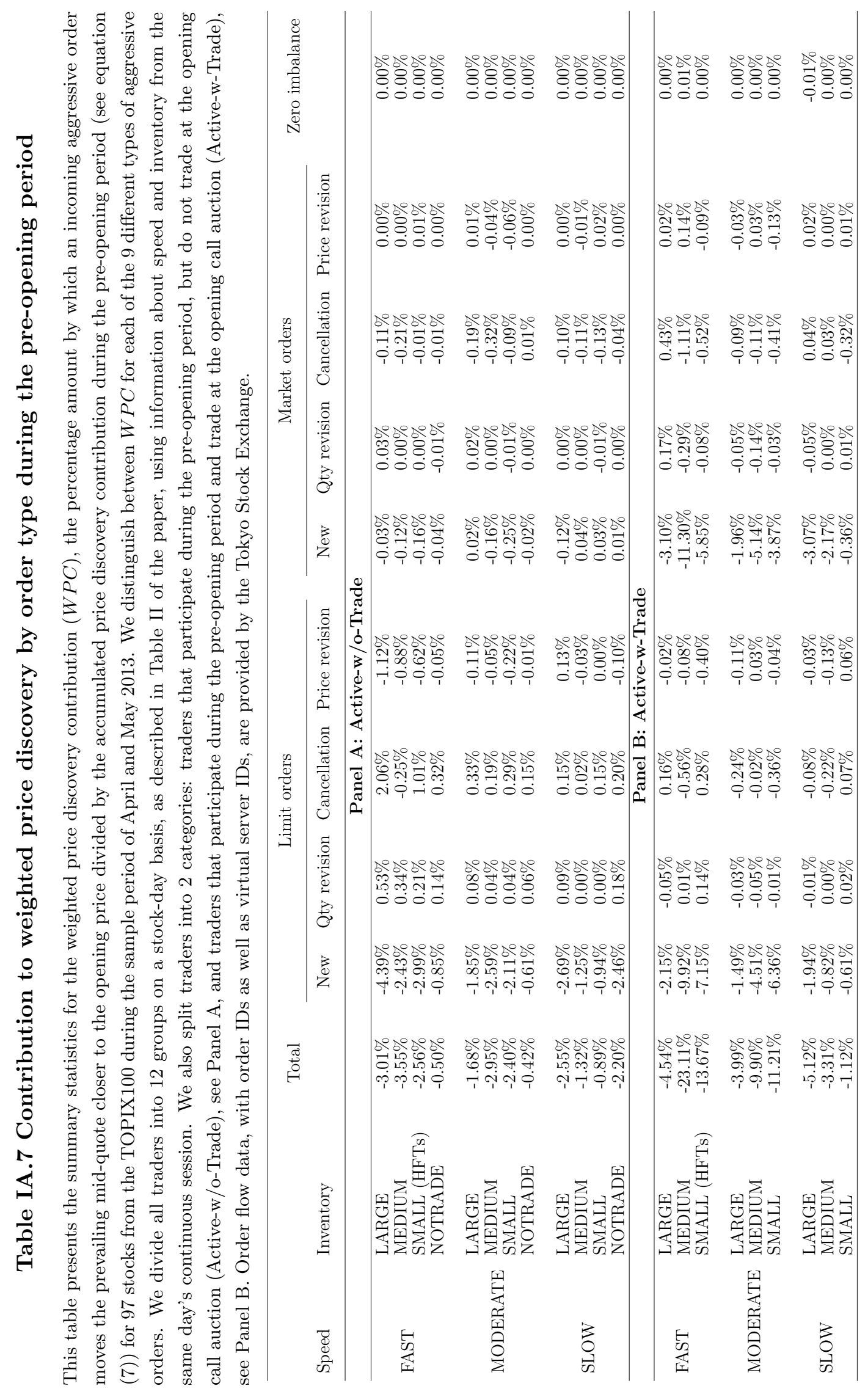




\section{G.4. Speed of price discovery}

One of the questions we aim to answer with this paper concerns price discovery. We have considered the pre-opening period without distinguishing the earlier part of the period from the minute just before the auction. However, the order submission activity during the pre-opening section varies as the auction time approaches. In particular, a detailed analysis of the order flow allows us to confirm that the number of order submissions rises right before the opening auction. For a detailed description of the the pre-opening order flow, including the quoting activity for 12 trader groups, see Section E of this Internet Appendix. Therefore, we look more closely at the movements in the pre-opening quotes between 8:10 and 9:00 am to determine how quickly the preopening quotes approach the opening price for the day, closer to the auction time. For this purpose, we report in Figure IA.6 the absolute value of the relative deviation of the quoted prices from the opening price for each stock, on each day, calculated using equation (6) from Section II.C.2 of the paper.

Figure IA.6 shows the median of the second-by-second movements in the pre-opening quotes, across the 97 stocks. 10 minutes after the start of the pre-opening period, the deviation is around $0.66 \%$. The deviation decreases slowly during the period from 8:10 to 9:00 am. 10 minutes before the opening auction, the deviation is around $0.39 \%$. This suggests that around $60 \%(=0.39 / 0.66)$ of the total price discovery during the pre-opening period excluding the first 10 minutes, occurs during the last 10 minutes. The deviation diminishes to $0.06 \%$ just before the opening time

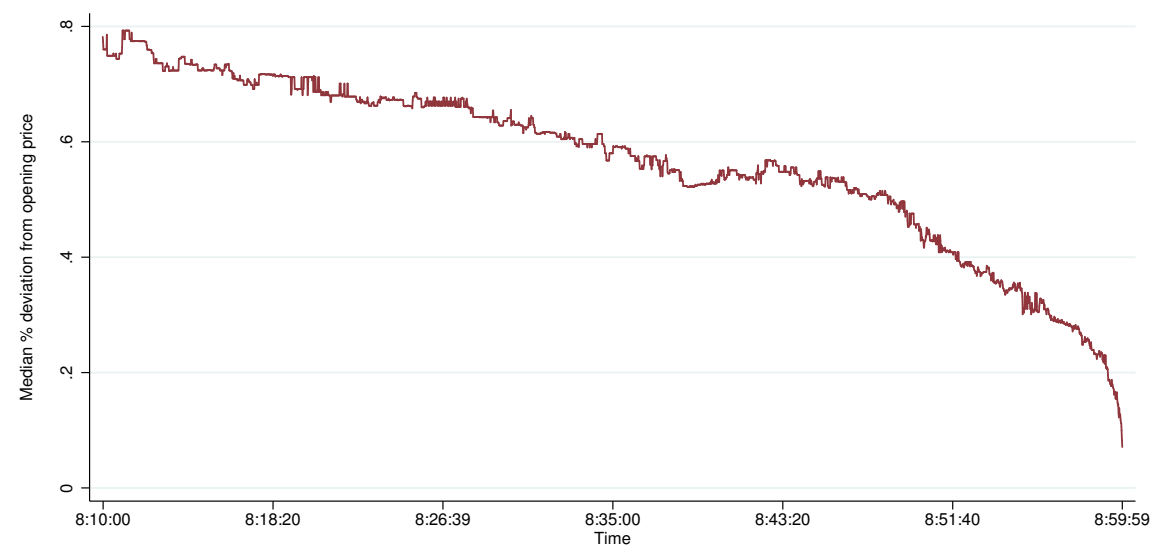

Figure IA.6. Deviation from the opening price. This figure shows the deviation of the pre-opening midquote from the opening price, computed for each second of the pre-opening period (8:10:00.000 - 8:59:59.999) for 97 stocks from the TOPIX100 during the sample period of April and May 2013. The deviation is defined as the percentage difference between the mid-quote, $M_{t, k}$, at time $t$ on day $k$, and the opening price, $O_{k}$, on day $k$, as defined in equation (6) of the paper. The deviation is computed per second per day per stock and then medians are calculated for each second. Order flow data, with order IDs as well as virtual server IDs, are provided by the Tokyo Stock Exchange.

In order to verify whether stocks with greater presence of HFTs exhibit different patterns of price convergence, we conduct the analysis in two steps. First, we investigate whether we observe a significant variation in the relative activity of different types of traders across stocks in terms of the proportion of aggressive order submissions. In particular, for each stock, we estimate the relative activity of each trader group as the number of aggressive quotes (quotes that could potentially have an impact on the quoted price) from each trader group relative to the number of aggressive quotes 
from all trader groups during the pre-opening period excluding the first 10 minutes, aggregated across stocks and days (see Table IA.8). FAST/SMALL (HFTs) exhibit wide variation in their activity from stock to stock for the pre-opening period excluding the first 10 minutes: from $5.80 \%$ to $58.65 \%$.

\section{Table IA.8 Aggressive orders across stocks}

This table provides summary statistics for the aggressive orders across stocks. We divide all traders into 12 groups on a stock-day basis, as described in Table II of the paper, using information about speed and inventory from the same day's continuous trading period. For each stock, we compute the proportion of aggressive orders (orders with the potential to impact the prevailing quotes) submitted by each group of traders relative to the total number of aggressive orders for a particular stock during the pre-opening period, excluding the first 10 minutes for April and May 2013 across 97 stocks from TOPIX100. Aggressive orders are defined as follows: (1) all market orders; (2) limit buy orders with a limit price greater than or equal to the prevailing best bid; (3) limit sell orders with a limit price less than or equal to the prevailing ask; (4) any orders submitted when best bid equals best ask. Order flow data, with order IDs as well as virtual server IDs, are provided by the Tokyo Stock Exchange.

\begin{tabular}{llccccccc}
\hline \multirow{2}{*}{ Speed } & Inventory & MIN & P5 & P25 & P50 & P75 & P95 & MAX \\
\hline \multirow{5}{*}{ FAST } & LARGE & $1.57 \%$ & $2.54 \%$ & $4.03 \%$ & $6.40 \%$ & $8.49 \%$ & $12.32 \%$ & $14.85 \%$ \\
& MEDIUM & $12.27 \%$ & $14.95 \%$ & $19.20 \%$ & $20.83 \%$ & $23.62 \%$ & $27.25 \%$ & $35.76 \%$ \\
& SMALL (HFTs) & $5.80 \%$ & $9.94 \%$ & $14.89 \%$ & $19.09 \%$ & $28.74 \%$ & $43.73 \%$ & $58.65 \%$ \\
& NOTRADE & $0.02 \%$ & $0.04 \%$ & $0.23 \%$ & $0.56 \%$ & $0.99 \%$ & $2.30 \%$ & $3.29 \%$ \\
\multirow{5}{*}{ MODERATE } & & & & & & & \\
& LARGE & $1.33 \%$ & $2.15 \%$ & $3.17 \%$ & $4.42 \%$ & $5.75 \%$ & $7.53 \%$ & $8.49 \%$ \\
& MEDIUM & $4.53 \%$ & $7.82 \%$ & $11.80 \%$ & $14.02 \%$ & $16.67 \%$ & $19.40 \%$ & $22.45 \%$ \\
& SMALL & $5.82 \%$ & $9.38 \%$ & $12.75 \%$ & $16.03 \%$ & $20.08 \%$ & $24.64 \%$ & $27.62 \%$ \\
& NOTRADE & $0.01 \%$ & $0.04 \%$ & $0.15 \%$ & $0.29 \%$ & $0.53 \%$ & $1.26 \%$ & $2.67 \%$ \\
& & & & & & & & \\
\multirow{5}{*}{ SLOW } & LARGE & $0.69 \%$ & $1.60 \%$ & $3.26 \%$ & $5.39 \%$ & $8.07 \%$ & $11.63 \%$ & $17.27 \%$ \\
& MEDIUM & $0.34 \%$ & $0.89 \%$ & $1.80 \%$ & $3.53 \%$ & $4.55 \%$ & $6.63 \%$ & $8.30 \%$ \\
& SMALL & $0.30 \%$ & $0.47 \%$ & $1.26 \%$ & $2.64 \%$ & $3.61 \%$ & $4.63 \%$ & $6.10 \%$ \\
& NOTRADE & $0.07 \%$ & $0.17 \%$ & $0.48 \%$ & $1.07 \%$ & $1.62 \%$ & $3.30 \%$ & $5.76 \%$ \\
\hline
\end{tabular}

Second, based on the distribution of the relative activity of the traders, we separate the 97 stocks from the TOPIX100 into two groups: stocks for which the activity of FAST/SMALL traders (HFTs) during the pre-opening period excluding the first 10 minutes crosses a threshold of $30 \%$ (18 stocks), and all other stocks (79 stocks). Figure IA.7 presents the median absolute deviation of the quoted price from the opening price, per second of the pre-opening period, and for the last 10 minutes of the pre-opening period. Note that, for stocks that pass the $30 \%$ threshold, the median absolute deviation is always smaller than it is for stocks that do not pass the threshold. However, immediately before the opening auction, the absolute deviation is approximately the same for both stock groups. The maximum gap between the two series is $0.20 \%$. During the last 10 minutes of the pre-opening period, the median gap size is around $0.10 \%$, except in the last couple of seconds, during which the gap closes rapidly due to the convergence of the absolute deviation to the opening price of the second group of stocks. According to these results, the presence of the FAST/SMALL traders (HFTs) improves the efficiency of the price discovery process. 
Panel A - Deviation for the entire pre-opening period for the two groups of traders

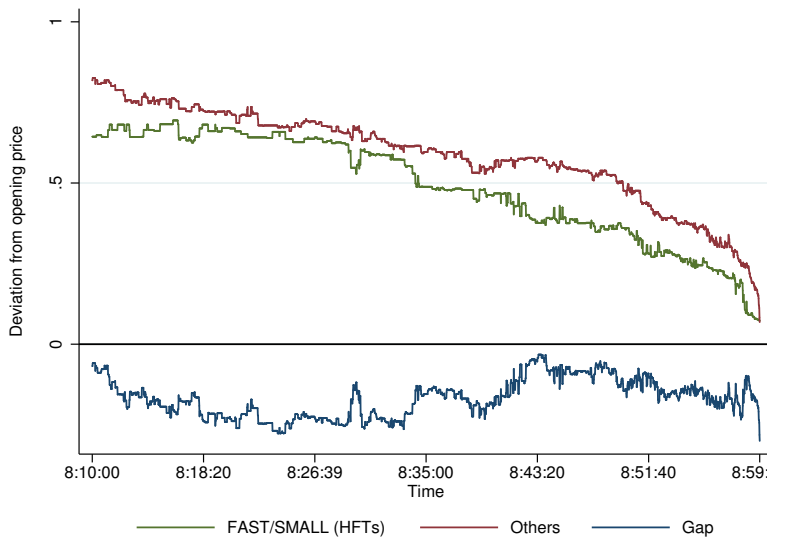

Panel B - Deviation for the last 10 minutes for the two groups of traders

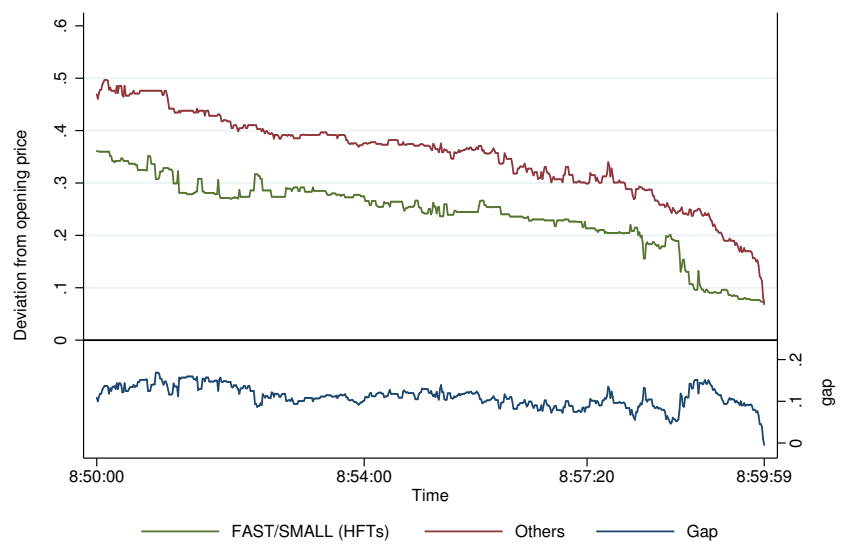

Figure IA.7. Comparison of the deviation from the opening price between stocks for which highspeed traders have different levels of participation. This figure shows, for two groups of stocks, the percentage deviation of the pre-opening mid-quote from the opening price, computed for each second of the pre-opening period (8:10:00.000 - 8:59:59.999), for 97 stocks from the TOPIX100, during the sample period of April and May 2013. We split stocks into two groups: the first group includes stocks for which aggressive activity by HFTs passes a threshold of $30 \%$ (18 stocks). The second group includes all other stocks (79 stocks). Panel A displays the deviation for the pre-opening period excluding first 10 minutes for the two groups of stocks, while Panel B displays deviations for the last 10 minutes of the pre-opening period. Order flow data, with order IDs as well as virtual server IDs, are provided by the Tokyo Stock Exchange. 


\section{H. Unbiasedness of the quoted price}

We test for the price efficiency of the pre-opening quotes across the two groups using an unbiasedness regression that has been widely used in the literature. ${ }^{4}$

This technique is widely applied to characterize the extent to which there is learning and price discovery in the pre-opening period. In the cited contribution, the proxy of the equilibrium price $v$ is usually the closing price of the day. We modify their framework for our purposes and estimate the following equation:

$$
\nu-E\left(\nu \mid I_{0}\right)=\alpha_{t}+\beta_{t}\left[P_{t}-E\left(\nu \mid I_{0}\right)\right]+Z_{t}
$$

where $\nu$ is the opening price (instead of the closing price used in Biais, Hillion, and Spatt (1999)), $P_{t}$ is the pre-opening quoted price, and $E\left(\nu \mid I_{0}\right)$ is the previous day's closing price. The distribution of the change in price, from the previous day's close to the quoted price, varies over time as the opening time approaches. The amount of noise in the quoted price is also likely to vary with time. In this spirit, we estimate the unbiasedness regression using the specification shown in equation (IA.2), for each 1-second interval and for each stock. If the pre-opening quoted price is an unbiased estimator of the opening price, the coefficient $\beta_{t}$ in the specification should be insignificantly different from 1 . We hypothesize that the earlier in the pre-opening period the coefficient $\beta_{t}$ equals 1 , the greater is the price efficiency of the pre-opening quote. We analyze the pattern of the value of the $t$-statistic, under the null hypothesis that $\beta_{t}$ is equal to 1 , over the pre-opening period. Figure IA.8 shows the $\beta_{t}$ estimates for each second during the pre-opening period. From Figure IA.8, we can see that $\beta_{t}$ steadily increases during the pre-opening period. However, $\beta_{t}$ becomes insignificantly different from 1 only seconds before the opening call auction.

Panel A - Beta coefficient

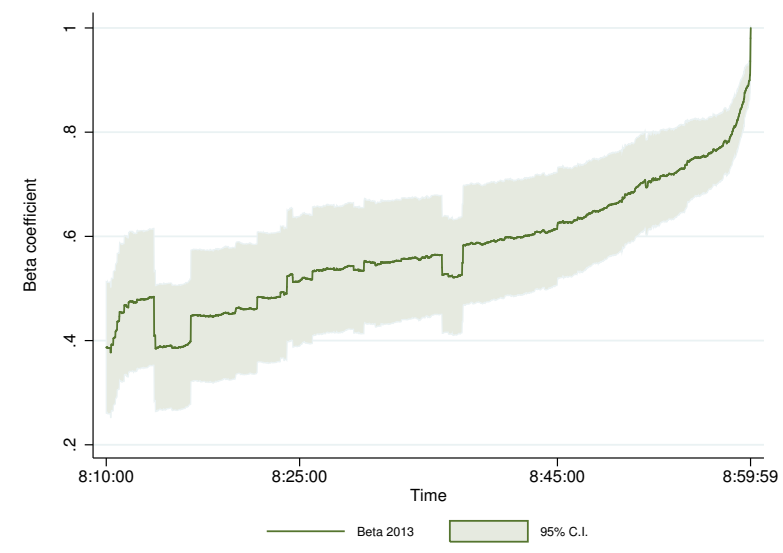

Panel B - T-statistic

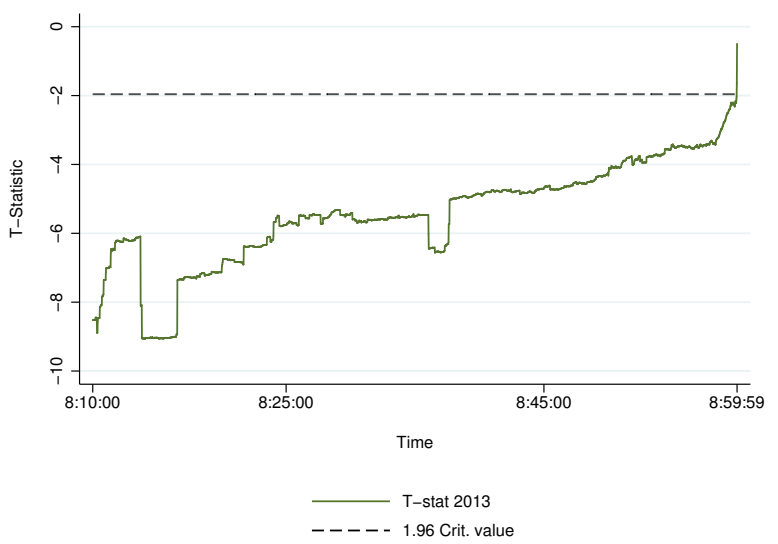

Figure IA.8. Tests of unbiasedness regressions of the pre-opening mid-quotes Using mid-quotes, we estimate equation (IA.2) every second during the pre-opening period (8:10:00.000 - 8:59:59.999) for 97 stocks from the TOPIX100, during the sample period of April and May 2013. The figures show the averages of the $\beta_{t}$ coefficients (Panel A) and the $t$-statistics under the null hypothesis that $\beta_{t}$ is equal to 1 (Panel B). The tick-by-tick data, time stamped to the millisecond, are obtained from the Thomson-Reuters Tick History Database.

\footnotetext{
${ }^{4}$ Among other papers that use an unbiasedness regression to investigate price discovery are Biais, Hillion, and Spatt (1999), Barclay and Hendershott (2003, 2008), Comerton-Forde and Rydge (2006), and Chakrabarty, Corwin, and Panayides (2011).
} 
Furthermore, there are remarkable cross-sectional differences. To exploit them graphically, we follow the same approach as in Section G.G.4. We divide the stocks into two groups based on the activity of FAST/SMALL traders (HFTs). Figure IA.9 shows the $\beta_{t}$ estimates and $t$-statistic under the null hypothesis that $\beta_{t}$ is equal to 1 for every second during the pre-opening period, for these two groups of stocks, for April and May 2013. Remarkably, the $\beta_{t}$ for stocks subject to high activity from the FAST/SMALL traders (HFTs) differs insignificantly from 1 during the whole pre-opening period. On the contrary, the $\beta_{t}$ for stocks subject to low activity from the FAST/SMALL traders (HFTs) increases slowly from 0.3 to 1 , and only becomes insignificantly different from 1 seconds before the opening call auction. Overall, these results are consistent with FAST/SMALL traders (HFT) improving price discovery during the pre-opening period.

Panel A - Beta coefficient for the two groups

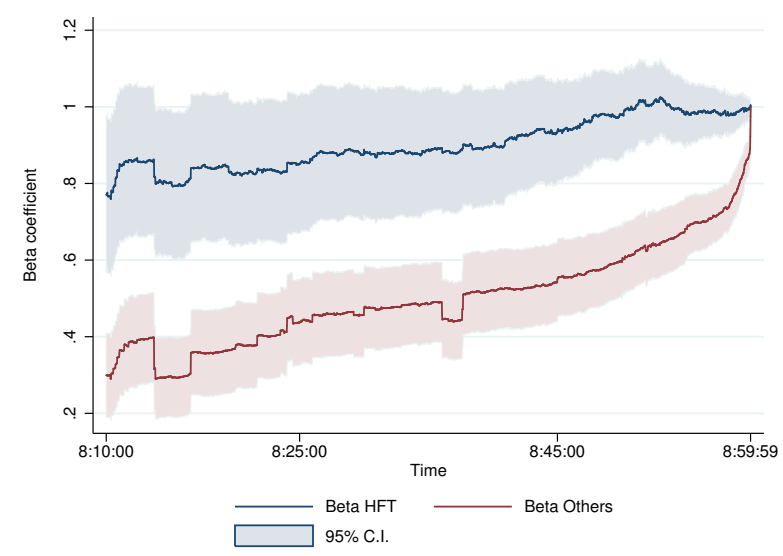

Panel B - T-statistic for the two groups

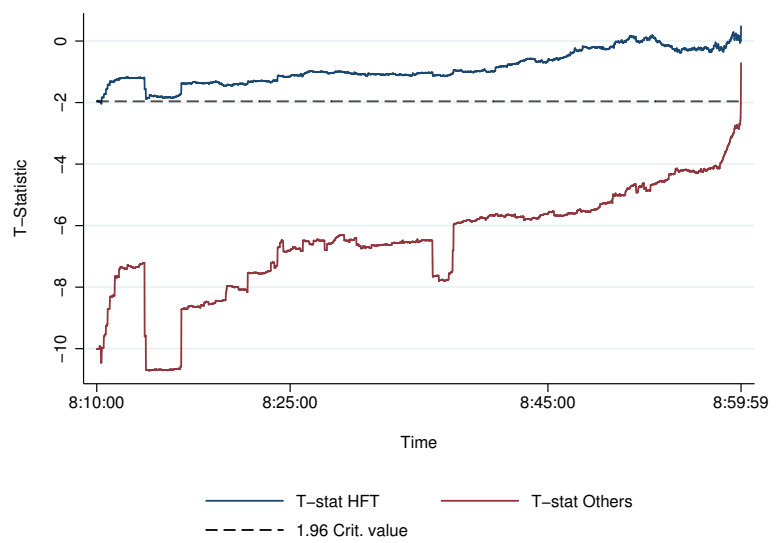

Figure IA.9. Comparison of the test of unbiasedness regressions between stocks with different levels of high-speed traders participation Using mid-quotes, we estimate equation (IA.2) at each second of the pre-opening period (8:10:00.000 - 8:59:59.999), for each of the 97 stocks from the TOPIX100 during the sample period of April and May 2013. We split stocks into two groups: the first group includes stocks for which aggressive activity by HFTs passes a threshold of 30\% (18 stocks). The second group includes all other stocks (79 stocks). The averages of the $\beta_{t}$ coefficients are shown in Panel A. Panel B shows the $t$-statistics under the null hypothesis that $\beta_{t}$ is equal to 1. The tick-by-tick data, time stamped to the millisecond, are obtained from the Thomson-Reuters Tick History Database. 


\section{References}

Amihud, Yakov, and Haim Mendelson, 1991, Volatility, efficiency, and trading: Evidence from the Japanese stock market, Journal of Finance 46, 1765-1789.

Barclay, Michael J, and Terrence Hendershott, 2003, Price discovery and trading after hours, Review of Financial Studies 16, 1041-1073.

Barclay, Michael J, and Terrence Hendershott, 2008, A comparison of trading and non-trading mechanisms for price discovery, Journal of Empirical Finance 15, 839-849.

Barclay, Michael J, and Jerold B Warner, 1993, Stealth trading and volatility: Which trades move prices?, Journal of Financial Economics 34, 281-305.

Berkman, Henk, Paul D Koch, Laura Tuttle, and Ying Jenny Zhang, 2012, Paying attention: overnight returns and the hidden cost of buying at the open, Journal of Financial and Quantitative Analysis 47, 715-741.

Biais, Bruno, Pierre Hillion, and Chester Spatt, 1995, An empirical analysis of the limit order book and the order flow in the Paris Bourse, Journal of Finance 50, 1655-1689.

Biais, Bruno, Pierre Hillion, and Chester Spatt, 1999, Price discovery and learning during the preopening period in the Paris Bourse, Journal of Political Economy 107, 1218-1248.

Brogaard, Jonathan, Björn Hagströmer, Lars L Norden, and Ryan Riordan, 2015, Trading fast and slow: Colocation and market quality, Review of Financial Studies 28, 3407-3443.

Cao, Charles, Eric Ghysels, and Frank Hatheway, 2000, Price discovery without trading: Evidence from the NASDAQ preopening, The Journal of Finance 55, 1339-1365.

Chakrabarty, Bidisha, Shane A Corwin, and Marios A Panayides, 2011, When a halt is not a halt: An analysis of off-NYSE trading during NYSE market closures, Journal of Financial Intermediation 20, 361-386.

Chakravarty, Sugato, 2001, Stealth-trading: Which traders' trades move stock prices?, Journal of Financial Economics 61, 289-307.

Comerton-Forde, Carole, and James Rydge, 2006, The influence of call auction algorithm rules on market efficiency, Journal of Financial Markets 9, 199-222. 
Duong, Huu Nhan, Petko S Kalev, and Chandrasekhar Krishnamurti, 2009, Order aggressiveness of institutional and individual investors, Pacific-Basin Finance Journal 17, 533-546.

Kirilenko, Andrei A, Albert S Kyle, Mehrdad Samadi, and Tugkan Tuzun, 2016, The Flash crash: High frequency trading in an electronic market, Journal of Finance, Forthcoming.

Ranaldo, Angelo, 2004, Order aggressiveness in limit order book markets, Journal of Financial Markets 7, 53-74.

Stoll, Hans R, and Robert E Whaley, 1990, Stock market structure and volatility, Review of Financial Studies 3, 37-71.

TSE, 2015, Tokyo Stock Exchange: Guide to TSE trading methodology.

Yamamoto, Ryuichi, 2011, Order aggressiveness, pre-trade transparency, and long memory in an order-driven market, Journal of Economic Dynamics and Control 35, 1938-1963. 


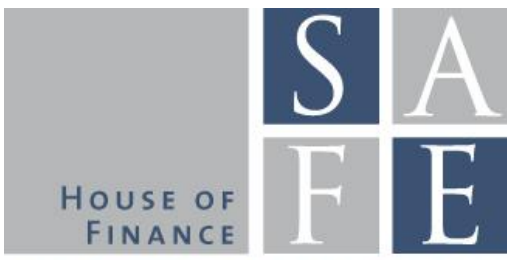

WORKING PAPER SERIES

\section{Recent Issues}

No. 143 Peter Gomber, Satchit Sagade, Erik Theissen, Moritz Christian Weber, Christian Westheide

No. 142 Nathanael Vellekoop

No. 141 Brigitte Haar

No. 140 Reint Gropp, Rasa Karapandza, Julian Opferkuch

No.139 Holger Kraft, Claus Munk, Farina Weiss

No.138 Mohammed Aldegwy, Matthias Thiemann

No. 137

Elia Berdin, Cosimo Pancaro, Christoffer Kok

No. 136

Matthias Thiemann, Mohammed Aldegwy, Edin Ibrocevic

No. 135

Douglas Cumming, Jochen Werth, Yelin Zhang

No. 134 Markus Kröll, Devesh Rustagi

No. 133 Markus Behn, Rainer Haselmann, Thomas Kick, Vikrant Vig

No. 132 Rainer Haselmann, David Schoenherr, Vikrant Vig
Spoilt for Choice: Order Routing Decisions in Fragmented Equity Markets

The Impact of Long-Run Macroeconomic Experiences on Personality

Freedom of Contract and Financial Stability through the lens of the Legal Theory of Finance

The Forward-Looking Disclosures of Corporate Managers: Theory and Evidence

Predictors and Portfolios Over the Life Cycle

How Economics Got it Wrong: Formalism, Equilibrium Modelling and PseudoOptimization in Banking Regulatory Studies

A Stochastic Forward-Looking Model to Assess the Profitability and Solvency of European Insurers

Understanding the Shift from Micro to MacroPrudential Thinking: A Discursive Network Analysis

Governance in Entrepreneurial Ecosystems: Venture Capitalists vs. Technology Parks

Got Milk? Motivation for Honesty and Cheating in Informal Markets: Evidence from India

The Political Economy of Bank Bailouts

Rent-Seeking in Elite Networks 